\title{
AN APPROACH TO MODELLING TREE ROOT ARCHITECTURE IN VIRTUAL URBAN GROWING CONDITIONS
}

\author{
by \\ Justin Miron, \\ Master of Landscape Architecture, University of Toronto, 2011 \\ Honours Bachelor of Arts (Magna cum Laude), York University, 2007
}

A Major Research Paper (MRP) presented to Ryerson University in partial fulfillment of the requirements for the degree of Master of Spatial Analysis (MSA) in the Program of Spatial Analysis.

Toronto, Ontario, Canada, 2017

(C) Justin Miron, 2017 


\section{AUTHOR'S DECLARATION FOR ELECTRONIC SUBMISSION OF A MRP}

I hereby declare that I am the sole author of this MRP. This is a true copy of the MRP, including any required final revisions.

I authorize Ryerson University to lend this MRP to other institutions or individuals for the purpose of scholarly research.

I further authorize Ryerson University to reproduce this MRP by photocopying or by other means, in total or in part, at the request of other institutions or individuals for the purpose of scholarly research. I understand that my MRP may be made electronically available to the public.

Justin Miron 


\section{Statement of Authorship and Co-Authorship}

Assistance with this research was provided by my advisor, Dr. Andrew Millward. If portions or the entirety of this Major Research Paper in published in a research journal or other publication, both I and Dr. Millward should be listed as co-authors, each with the contributions noted below:

\section{First author (Miron)}

Study design and implementation;

Technical root architecture simulation implementation in MATLAB;

Simulation results analysis and primary writer of the research paper.

\section{Second author (Millward)}

Assistance with study design and implementation;

Assistance with requisition of outside technical expertise in MATLAB;

Editorial support for the manuscript. 


\section{An Approach to Modelling Tree Root Architecture in Virtual Urban Growing Conditions}

Justin Miron

Master of Spatial Analysis (MSA), 2017, Program in Spatial Analysis

Ryerson University

Understanding the architecture of tree roots is an important component of urban forestry management practice. Tree roots are structurally and functionally important to the survival of trees, and this can be even more so in urban environments where underground space for roots is limited. Tree root architecture models can provide a complementary approach to traditional onsite field investigation methods. Root architecture models are unique in that they can simulate the spatial arrangements of root system structure explicitly, and allow investigators to create hypothetical simulations to test their assumptions about what may be driving root growth. The use of root architecture models in the literature is extensive and may be applied in diverse streams of investigation, but their application to tree root systems is less common. This research demonstrates a root architecture model, Rootbox, as a case study in the application of plant architecture models to simulate tree root growth in urban conditions. Model parameterization was based on conformity of root simulations to tree root architecture reported in the literature. The model is deployed in four hypothetical urban soil scenarios, which are representative of planting sites commonly observed in urban settings. The analysis demonstrates that plausible tree root system architectures - specifically, commonly observed growth attributes - can be produced by Rootbox, but only after several adaptive changes to both the source code/model design are made. Custom soil models can integrate with the simulation to represent urban conditions by modifying both the growth direction and elongation of portions of the root architecture, and thus offer greater control over the output architecture. Rootbox offers a flexible method of simulating the architecture of tree root systems, but further research should focus on optimizing the model's parameters and functions to enable greater user control over model output. 


\section{$\underline{\text { Acknowledgements }}$}

I would like to thank my advisor, Dr. Andrew Millward, for his continual support and encouragement during the course of this MRP's development; and Dr. Stephanie Melles, who provided valuable comments and suggestions during review.

Also, I would like to thank UFRED members Vadim Sabetski and Christopher Scarpone, who both provided valuable and timely commentary that helped shape the project's direction, and Dr. John Enright, who provided some crucial technical assistance in MATLAB.

Finally, I would like to thank my husband, Jay Walker, and my family for their encouragement and patience during the course of the project. 


\section{Table of Contents}

Author's Declaration for Electronic Submission of a MRP

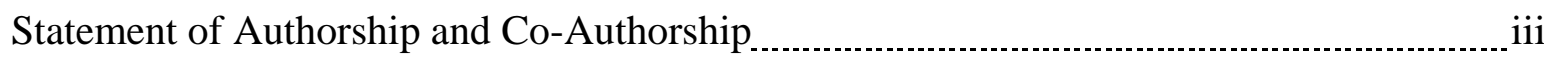

Abstract

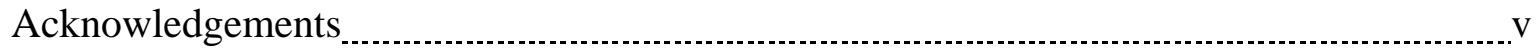

Table of Contents

List of Tables

List of Figures

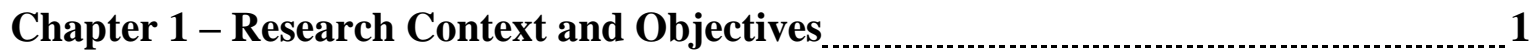

1.1 - Research Objectives

1.2 - Structure of Major Research Paper

Chapter 2 - Research Background

2.1 - Tree Root System Development in Urban Conditions _....................................... 5

2.2 - Tree Root Detection in Field Investigations

2.3 - Tree Root System Models

2.4 - Setting the Parameters of Root Architecture Models $\ldots$

2.5 - Relevant Morphological Characteristics of Tree Root Systems _ 10

2.6 - Combining Root Architecture Models and Soil Models _..................................... 12

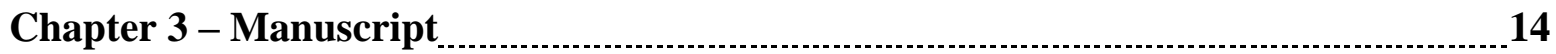

3.1 - Introduction 14

3.2 - Methods

3.2.1 - Description of Rootbox

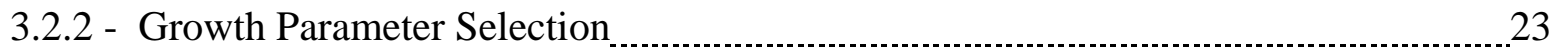

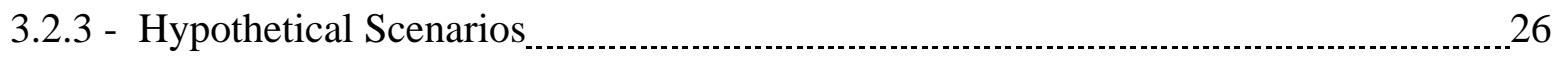

3.3 - Results

3.3.1 - Scenario 1 - Base Grid 41

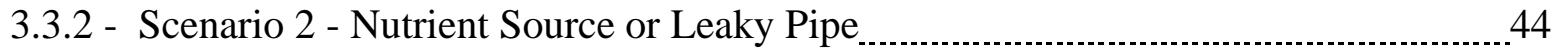

3.3.3 - Scenario 3 - Sidewalk (Physical Barrier) Underlaid with Compacted Soil _............. 48

3.3.4 - Scenario 4 - Weighting the Basic Grid Based on In-Situ Knowledge of Root Location $\ldots$ 
3.4 - Discussion

3.4.1 - ZRT and Root Diameter

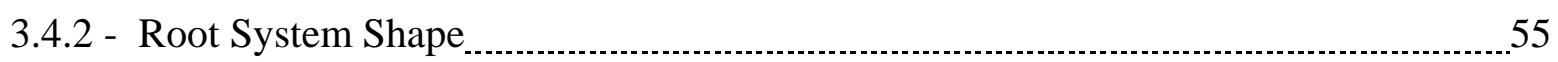

3.4.3 - Simulating Urban Conditions with Soil Grids _............................................. 57

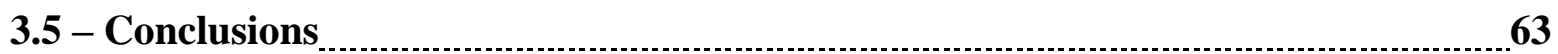

Chapter 4 - Limitations, and Opportunities for Further Research

4.1 - Research Limitations $\ldots$

4.1.1 - Rootbox as a Predictive Model of Actual Tree Root Systems _........................... 65

4.1.2 - Sensitivity Analysis and Model Parameter Optimization $\ldots$

4.1.3 - Incorporation Of Fine Roots, Root Thickening and Root Turnover _.......................... 68

Appendices $\quad 71$

References $\quad 75$ 


\section{$\underline{\text { List of Tables }}$}

Table 3.1 - Rootbox parameters governing root growth 20

Table 3.2 - Scenario 1 average root diameter vs distance measurements _..........................44

Table 3.3 - Scenario 2 average root diameter vs distance measurements _ _......................... 48

Table 3.4 - Scenario 3 average root diameter vs distance measurements $\ldots \ldots \ldots \ldots \ldots \ldots \ldots \ldots \ldots$ 


\section{List of Figures}

Figure 3.1 - Schematic depiction of_parameters governing root growth and branching structure in Rootbox

Figure 3.2 - Schematic depiction_of successor-orders that comprise the final architecture simulations used in this research 22

Figure 3.3 - Surface view of Scenario 1 soil grid 28

Figure 3.4 - Profile view of Scenario 1 soil grid 29

Figure 3.5 - Surface view of Scenario 2 soil grid 31

Figure 3.6 - Profile view of Scenario 2 soil grid 32

Figure 3.7 - Surface view of Scenario 3 soil grid 34

Figure 3.8 - Profile view of Scenario 3 soil grid 35

Figure 3.9 - Surface view of Scenario 4 soil grid 37

Figure 3.10 - Profile view of Scenario 4 soil grid 38

Figure 3.11 - Surface distribution of Scenario 1 model output 41

Figure 3.12 - Profile view of Scenario 1 model output 42

Figure 3.13 - Averaged root depth of Scenario 1 model output 43

Figure 3.14 - Surface distribution of Scenario 2 model output 45

Figure 3.15 - Profile view of Scenario 2 model output 46

Figure 3.16 - Averaged root depth of Scenario 2 model output 47

Figure 3.17 - Surface distribution of Scenario 3 model output 49

Figure 3.18 - Profile view of Scenario 3 model output 49

Figure 3.19 - Averaged root depth of Scenario 3 model output 50

Figure 3.20 - Surface distribution of Scenario 3 model output 52

Figure 3.21 - Profile view of Scenario 4 model output 52

Figure 3.22 - Averaged root depth of Scenario 4 model output 53 


\section{Chapter 1 - Research Context and Objectives}

Understanding the architecture of tree roots is an important component of urban forestry management. Tree roots are structurally and functionally important to the survival of trees, and this is even more so in urban environments where underground space for roots is limited. Yet, tree roots are difficult to investigate because they are (predominantly) located under-ground and cannot be observed directly without performing an invasive - and often destructive - excavation. As an alternative, tree roots can be detected using non-invasive methods like ground penetrating radar (GPR), to map the locations of coarse roots. However, even this approach, while not invasive, is still potentially expensive, laborious and time consuming, and problems persist with root-identification accuracy and the ability to comprehensively document the architecture of a tree's root system.

A potential complementary approach to on-site field investigations is the use of a tree root architecture model to aid in the anticipation of how root systems can or will develop and grow in three-dimensional space. Tree root architectural models simulate an explicit - but hypothetical three-dimensional root system that accounts for the spatial positions of all roots and their attributes, such as diameter, length, growth direction, age and position in the hierarchy of the root branching structure. Architectural models are spatial models that can produce potentially large quantities of information about how a root system may develop per a set of governing assumptions. In the literature, root architecture models are employed where there is a requirement to know or predict not only the location of roots but also to understand root system geometry, i.e. the size and shape of roots, and topology, i.e. the relationship of individual roots to one another and to the plant as a whole. Root architecture models in these cases may provide an armature upon which further plant functions can be simulated. For example, a root architecture model can simulate the branching network, and this network can then be used as input into a water- or nutrient- uptake model. Due to their capacity to generate hypothetical root systems, architectural models are well-suited to testing how prevailing understandings of root system morphology and function "play out" on hypothetical sites, and can therefore not only aid in onsite root detection efforts, but can also play an important role in site design for new tree installations. 
Plant root architecture models are typically implemented within a software framework, and there are a variety of model software that can be used to run a simulation. Some of these models are highly specialized and inflexible in their design, and often are used to solve highly specific research problems where plant root architecture plays a significant part in the analysis. They typically force the investigator to modify their study approach to conform to the model's rules of practice and implementation. Most research that uses plant root architecture modelling focuses on non-tree plant species, but plant root architecture models also have applicability to tree root systems. However, it can be difficult to model the architecture of tree root systems because these form over both multi-annual time periods and the underlying growth mechanisms operate at multiple spatial and temporal resolutions; they often exhibit highly unique spatial morphologies, especially in the highly heterogeneous environments encountered in urban contexts; and it is difficult to acquire complete tree root architecture data upon which the model's accuracy can be compared.

In this study, the MATLAB-based Rootbox model (Leitner et al., 2010a; University of Vienna, 2017) is used to simulate the morphology of tree roots in four hypothetical urban conditions. Rootbox is implemented in an "open" - i.e., modifiable - software framework that is not fully outfitted with advanced plant simulation functions, but it can implement several functions that are core to any root architecture model. The validity of a selection of model parameters is tested by evaluating how they reproduce commonly reported morphological characteristics of tree root systems, including the commonly observed zone of rapid taper (ZRT) and the intense branching and growth of woody roots near the tree's base. The effects of urban conditions are tested in four scenarios by simulating the root system's growth in four alternative simulated urban soil environments. The purpose of the testing is to provide a demonstration of the use of Rootbox in practice of simulating hypothetical tree root systems, to facilitate a discussion of how the tool potentially can have use in field investigations of urban tree root systems.

Because root architecture models are programmed to behave under certain assumptions (parameters) in hypothetical situations, they are not meant to predict or match empirical tree root architecture. Nonetheless, further research may investigate ways to optimize parameters and 
overall model design to better anticipate tree root architecture on real sites and to make such models indispensable prediction tools that aid in urban tree investigations and management.

\section{1 - Research Objectives}

The following research aims to answer three questions:

1) Can Rootbox, a MATLAB-based plant root simulator, be used to generate realistic plant root architectures that reproduce general morphological traits commonly observed in tree root systems?

2) Can the effects of urban site conditions on the development of a tree's root architecture be accounted for in the model?

3) Is Rootbox a predictive model that can use empirical data inputs to simulate the likely tree root architecture on real-world sites more accurately? If not, can it be extended to be a predictive model?

Rootbox will be used to model a hypothetical tree root system under four scenarios representative of commonly occurring sub-surface urban growing conditions. This demonstration will allow Rootbox's potential utility and challenges as a tree root architecture model to be assessed and discussed from the perspective of the needs of arborists and landscape architects as potential users.

\section{2 - Structure of Major Research Paper}

Chapter 2 (following), Research Background, provides a literature review that discusses the difficulty of both managing and investigating urban tree roots and the approach of root architecture modelling and its practical use, including the selection of parameters and implementation alongside soil models.

Chapter 3, Manuscript, is presented as a manuscript and follows the guidelines specified for consideration for publication in the journal, Landscape and Urban Planning. It provides a concise Introduction to the research background and study objectives; it details the 
methodological approach in the Methods section; a description of the study's results in Results; a discussion of the results and their broader implications, including the study's limitations, for landscape and urban planning in Discussion, and a concluding summary in Conclusions.

Chapter 4, Limitations, and Opportunities for Further Research, details limitations of the research as well as recommended future research that may overcome these limitations.

Detailed parameter value selections are in Appendix A. An example of soil grid values is included in Appendix B. Reference MATLAB code for implementing a soil grid in MATLAB is included in Appendix C. 


\section{Chapter 2 - Research Background}

\section{1 - Tree Root System Development in Urban Conditions}

Urban soils impact the development and spatial arrangement of tree root systems in ways that are not well understood (Day et al., 2010; Day \& Wiseman, 2010). The primary functions of roots are to provide structural anchorage for the tree (Coutts, 1987; Danjon et al., 2008; Danjon et al., 2005; Gregory, 2006; McIvor et al., 2008) and to forage for nutrient and water resources in the soil (Gregory, 2006; Murray, 2011). While the phases of root development during the life history of the plant are under genetic control, the soil environment, which is typically found to have heterogeneously distributed resources, will moderate the spatial arrangement of roots and, moreover, individual tree root systems are impacted by the unique conditions of the site (Gregory, 2006; Forde \& Lorenzo, 2001). Conversely, the spatial arrangement and functioning of roots alters the soil environment (Gregory, 2006). The physical site conditions, and the highly heterogeneous nature of urban soils, often constrain the development of roots in various ways (Giuliani, et al., 2015; Nicoll \& Armstrong, 1998). Urban soil conditions are frequently hostile environments for root system health and, correspondingly, overall tree condition (Hawver \& Bassuk, 2006). Harsh growing conditions are a result of: compacted soils, which mechanically impede root growth (Alameda \& Villar, 2009; Alberty et al., 1984, Ampoorter et al., 2011; Page et al., 2015); minimal soil volume and associated alterations to soil composition and texture, which limit plate access to water, oxygen and nutrients necessary for survival, as well as constraining the size of the overall root system (Brunner et al., 2015; Blume, 1989; Buhler et al., 2017; Urban, 2008).

Coarse, woody roots are the result of fine, non-woody roots that have undergone secondary thickening (Fahey et al., 1994; Nygren et al., 2009). Fine, non-woody roots, are the roots that acquire nutrient and water resources, and their distribution over time impacts the eventual spatial arrangement of the system of coarse roots. Most fine roots have short life spans (Brunner et al., 2015; Day et al., 2010; Coutts, 1987), and tend to grow and die rapidly in response to changing soil conditions. Some fine roots persist and eventually become perennial and, as a result, their spatial arrangement becomes less reactionary to site conditions (Coutts, 1987; Nygren et al., 2009). The perennial arrangements of coarse roots are highly dependent on the small-temporal resolution of the life history of fine roots, often driven by changing soil conditions (Clausnitzer 
et al., 1994). By implication, persistent soil conditions, such as mechanical impediments like compaction, will have a definitive and profound effect on the spatial arrangements of the root system over longer periods of time (Coutts, 1987). For a full understanding of how roots systems develop and disperse in specific urban sites, and predicting how they will do this in the future, understanding the history of the site over both short and longer time periods (Mulia et al., 2010) is critical. However, in practice this is very difficult to achieve (Day et al., 2010; Dunbabin, et al., 2013; Pagès et al., 2004).

Anticipating the likely spatial distribution and arrangement of a root system on a specific urban site is important for effective urban forest management and tree protection (Day \& Wiseman, 2010). Estimations of root spread are used to determine the extent of the surface around the tree that should be protected on sites where soil disturbance is planned. In standard landscape architecture and civil engineering manuals, alteration of the soil grade within the drip-line - i.e. the growing medium below the tree and bounded by the extent of the tree canopy - is not recommended because it is here where the most important tree roots are considered to exist (Strom, 2009). Tree protection zones (TPZ) are typically erected to protect the root system from disturbance by designating an area up to a given distance from the tree as an area where soil disturbance is not permitted. The TPZ extents are established per the size of the tree, measured by tree trunk diameter at breast height (DBH) (City of Toronto, 2016). Day et al., (2010) argue that there is a strong relationship between DBH and root system spread within literature that accounts for both measures, and that site assessments that estimate the spread of a root system by referring to the $\mathrm{DBH}$ are reasonably reliable. Estimations of root system depth are more difficult in practice because it is a highly variable tree attribute among sites (Jackson et al., 1999; Stone \& Kalisz, 1991), and the depth habits of urban trees remains poorly understood (Day et al., 2010).

Generalized relationships between root spread and the size of the tree are both useful to practitioners estimating the location of roots underground and disadvantageous because they typically do not account for site specific influences on the spatial arrangement of the root system. Root systems often spread asymmetrically around a tree (Day et al., 2010) and this pattern is even more likely in urban sites as the root system must contend with anthropogenic obstacles and impediments. 


\section{2 - Tree Root Detection in Field Investigations}

Root systems are complex components of a plant organism that, in most plants, are located below ground and are, therefore, difficult to study (Amato et al., 2008; Lontoc-Roy et al., 2006). Most traditional methods of field investigation of tree roots are destructive and involve manually digging up portions of the root system, which is resource intensive and can sometimes kill the tree (Amato et al., 2008; Danjon et al., 2008). Additionally, such methods rarely allow investigators to observe the entirety of the root system and its structure. Much care must be taken to ensure that the mechanical exhumation does not alter the root system, or uncertainties will arise from attempts to generalize the analysis of tree roots in situ (Gregory, 2006). From the perspective of management and protection of existing trees, it does not makes sense to daylight tree roots and, in the process, kill a tree when the goal of excavation is to better understand how to protect the tree.

Non-destructive methods exist to detect the location (and other attributes) of tree roots, such as Ground Penetrating Radar (GPR). Stokes et al. (2002) suggest that GPR has been successful in the detection of the location of large (coarse) tree roots in non-urban environments, but in urban environments, GPR has had difficulty in differentiating between roots and some forms of urban infrastructure, such as pipes and cable (Stokes et al., 2002). GPR approaches also have difficulty accurately locating roots that are imaged at angles that approach parallel to the scan line, as well as detection of large roots in the vertical plane (Stokes et al., 2002). While GPR can be used to detect the location of course roots, its ability to generate detailed and accurate models of root architecture is an active area of research (Wu et al., 2014).

Root detection results from non-destructive methods like GPR and others (see Amato et al., 2008) are promising for management and design professionals; methods for improving the accuracy of detection are contining to be developed (Guo et al., 2013). Moreover, GPR root detection data can provide a base set of data for input into a root growth model (Wu et al., 2014). GPR detection is rapid (Stokes et al., 2002), and can be completed on a single root system in timeframes that could complement development of engineering or landscape architectural site tree protection plans (City of Toronto, 2016). However, it is potentially still expensive and labour intensive to implement, especially on many sites. In addition, because GPR is used to 
locate existing roots, it is by implication unable to anticipate future or hypothetical growth patterns.

\section{3 - Tree Root System Models}

A potential alternative to non-destructive detection techniques is the use of plant growth models to simulate root system growth. Plant growth models can be used to simulate various structural and functional characteristics of a root system (Fourcaud et al., 2008). These characteristics can then be used as a complement to site detection methods, depending on the specific focus of investigation. Models are often used heuristically - i.e. to test different hypothetical growth scenarios, rather than to predict the growth of a specific root system (Dunbabin et al. 2013; Lopez et al., 2011). However, they may be used to predict the behaviour of individual plant root growth under specific conditions (Leitner et al., 2010c; Walk et al., 2006). In this way, the use of plant root growth models may facilitate tree management planning and design by enabling the creation of scenarios that allow researchers to test assumptions of how tree roots spread and arrange themselves spatially and function underground (Pagès et al., 2004; Tobin et al., 2007), and also, depending on the design of the model, may be used to predict the locations and growth of the actual root systems.

Plant root simulations are of two generalized types: density-based models and architecture models (Gregory, 2006). Density-based models model the spatial arrangement of root density, irrespective of architectural or morphological characteristics like root branch order, size, orientation and direction, and spatial arrangement (Kaligiros et al., 2016). Density-based methods can take a variety of approaches. For instance, Heinen et al. (2003) model the growth of a root system as a process of diffusion of root length density. Reddy et al. (2001) model root spread as a convective-dispersive process. Dupuy et al., (2005) model the growth of a root system as a branching density function.

Architecture models simulate the explicit morphological characteristics of a root system in threedimensions (Dunbabin et al., 2013; Fourcaud et al., 2008; Leitner et al., 2010a; Pagès et al., 2014). The primary output of a root architecture model is an explicit three-dimensional digital representation of the spatial arrangement and location of roots and, depending on the model's 
complexity, their components (e.g. it stores where all the root tips are), their individual geometries (e.g. diameter and length), and their (topological) relationships to other parts of the root system (e.g. whether a root is a terminal member or whether it is an initial basal root). Architecture models are often computationally expensive because of the spatial complexity of the root systems they are modelling. However, these models have been used successfully to simulate root system architecture at different resolutions, from root hairs (Lenk et al., 2014) to coarser roots (Mulia et al., 2010). Modelling may be conducted at differing resolutions to reduce computational requirements (Leitner et al., 2010c). Architectural models have also been used alongside density-based models to reduce computational requirements while also preserving the capacity to model complex root-environment relationships (Dunbabin et al., 2013; Dupuy et al., 2005; Leitner et al., 2010c; Mulia et al., 2010).

Root architecture models can vary in their focus. Some architecture models are structural, and focus on the creation of the three-dimensional geometries only (Pagès et al., 2014). Other models explicitly include algorithms for modelling root functions, such as water and nutrient uptake; these models require the spatial location of root geometry to be considered explicitly in order to account for processes such as, for example, water movement through a root network (Couvreur et al., 2012; Clausnitzer et al., 1994). Root architecture models that include, or provide a basis for, plant function modelling are often referred to in the literature as functional-structural models, because they simulate the interaction of geometric as well as biological attributes of the plant with the environment (Godinet al., 2005; Tobin et al., 2007). Every root architecture model will at its most basic include routines for simulating a base three-dimensional geometry (Fourcard et al., 2008; Tobin et al., 2007) but can usually be upgraded to a functional-structural model by the addition of new software algorithms or, by using the three-dimensional output as an input to an environmental interaction model (Leitner et al., 2010b).

\section{4 - Setting the Parameters of Root Architecture Models}

As a methodological approach, root architectural models are useful primarily for the creation of three-dimensional "mock ups" or virtual experiments, which aid in the testing of hypotheses about how root systems develop under specific and varying circumstances (Fourcaud et al., 2008). In all cases, root architecture models must be provided with input parameter values from 
which the modelling framework (i.e. software) will procedurally generate an architecturally "realistic" morphology (Leitner et al., 2010a; Pagès et al., 2014). Parameter values moderate procedural development rules - which typically are computer-graphics script - and these generate the three-dimensional form (Leitner et al., 2010a; Nygren et al., 2009; Runions et al., 2007; Stava et al., 2014; Xu et al., 2012). For example, procedural rules will instruct a virtual root to extend by a distance increment defined by an input parameter, and terminate extension after a length determined by a length parameter. Different parameter values are often set for different root "types" (Zobel \& Waisel, 2010), allowing for a hierarchy of roots to be developed (e.g. by size and order of branching).

Crucially, parameter values are determined by a process of fitting to architectural data from realworld root systems observed in the field, or from published research. Data for fitting is acquired from field studies that explicitly measure architectural attributes (Garré et al., 2012), such as rate and angle of branching; or from a process of inverse modelling, which automatically generates parameter values from input three-dimensional data (Stava et al., 2014). This process can involve performing a sensitivity analysis to determine which architectural parameters have importance to making realistic (or "optimized") architecture models (Dunbabin, 2007; Garré et al., 2012; Ma et al., 2008; Pianosi et al., 2016). Data-fitting also requires a large sample from a variety of individual plant root systems to establish dominant or common architectural characteristics, which can prove practically difficult in the case of tree root systems, and especially those in urban conditions (Stokes et al., 2002; Tobin et al., 2007). If there is a lack of data, results from published research of tree root morphology can be used as the basis for estimating parameter values (Dunbabin et al., 2013). Development of a root architecture model that can accurately simulate root architecture on a real-world site will depend on coupling to empirical field measurements (Garré et al., 2012)

\section{5 - Relevant Morphological Characteristics of Tree Root Systems}

For this project, input parameters to the Rootbox model are based on reference to literature sources that describe the root architecture characteristics of a mature tree. Danjon et al. (2008) Stokes et al. (1996) and Coutts (1987) provide the primary informative sources for final parameter selection. Parameter selection is based on how well the final values produce tree root 
systems that conform to generalized patterns commonly observed in field studies of excavated tree root systems. Parameter selection is not meant to reproduce a tree root system observed on a real-world site.

Coutts (1987) and Stokes et al. (1996) suggest that overall root system morphology can be divided into three general categories: heart, lateral, and tap-root systems. This taxonomy was originally developed by Busgen et al. (1929; in Stokes et al., (1996)). In heart systems, coarse roots tend to occur and branch heavily under the base of the tree, but also spread horizontally in the top $50 \mathrm{~cm}$ of soil. Thus, heart root systems combine roots that grow vertically or semivertically, and horizontal roots. Both lateral and tap-root systems take either, but not both, of these approaches, but will not be discussed here.

In this research, parameters are selected that best reproduce the qualitative description of a heartsystem. In Rootbox (and in other plant root architecture models) a heart-system can be reproduced by carefully setting branching angle-, branching rate-, and branching distancecontrolling (i.e. length at which branching may occur and length between branches) parameters. Branching (or root initiation) angles tend to be oriented both vertically or horizontally at an area just below the stump, and the number of branches in this area is higher than in other, more distant parts of the root system (Coutts, 1987). The number of large woody roots that extend at horizontal angles from the base of the tree ranges between 1-10, depending on the species (Coutts, 1987); in Rootbox, the number of large, first-order roots is set to six. Additionally, root branching tends to occur on evenly-spaced points along the parent root (Coutts, 1987; Henderson et al., 1983); in Rootbox, successor branching is automatically evenly distributed along the length of the branching zone.

Initial branching angle has a profound effect on the ultimate vertical/horizontal orientation of roots (Coutts, 1987), but both coarse and fine roots of some tree species will tend to grow closer to the surface than they will vertically (Coutts et al., 1991; Coutts, 1989). The tendency for tree roots to grow closer to the surface (or on a horizontal angle from the base of the tree) is implemented in Rootbox by setting branch angle parameter values that favour horizontal angles, and by the addition of a directional bias towards the top $10 \mathrm{~cm}(0.1 \mathrm{~m})$ of the soil surface.

Rootbox parameters are selected to reproduce a realistic zone of rapid taper (ZRT). Trees exhibit a ZRT, which describes the reduction in root diameter over a short distance (usually 1-2m) from 
the base of the tree (Day et al., 2010). The ZRT has varying definitions, however, and this study uses the definition proposed by Danjon et al. (2005) to set root diameter parameters for several root types.

The collected literature above paints a picture of a root system architecture that informs the selection of Rootbox parameters. The architectural characteristics are general enough to apply to several tree species that could be found growing in urban environments. In practice, the selection of parameters using literature is more an art than it is rigorously objective, but nevertheless may enable investigators to make a quick appraisal of whether the parameters are "realistic" in comparison to field experience.

\section{6 - Combining Root Architecture Models and Soil Models}

The development of a tree root system architecture on real sites is highly influenced by the local soil environment. This is even more the case in urban growing sites, where more extreme soil heterogeneity is likely. Three-dimensional soil models can be used in conjunction with an architectural model to simulate soil effects on root system development by modifying the input architectural parameters of the architectural model at specific spatial locations. Soil models typically use gridded cells (i.e. voxels) (Jourdanet al., 1997) or a three-dimensional grid of points, that encode values that are accessed by the modification functions. For instance, an area of compaction can be simulated by modifying (reducing) the parameter value that controls the extension rate for the root tip, or by constraining the direction of the extending root tip, when the root tip reaches that grid point. The soil model in this case is not expressing real-world units of compaction, like bulk density or mechanical resistance, but is providing a spatially-explicit set of values that work with model software functions to express how root extension should be reduced as the roots move and grow into areas of high (or low) compaction. The values are a translation of measurements of real-world phenomena into effective values that have meaning within the framework of root system architecture. Density-based models, for instance, may translate such values differently. The translation methodology is potentially problematic, because the model framework may not capture real biological processes or environmental interactions but may only induce the architecture to mimic real world root architecture (Fourcaud et al., 2008; Marks et al., 2006). An example of a point grid approach is detailed in Clausnitzer et al. (1994), where bulk 
density and water uptake functions are encoded into a finite element grid of point locations that primarily control root elongation. Where soil compaction levels exceeding a specific tolerance are encountered by roots, their growth rate slows in a way that is commensurate with real-world measurements of how extension rates reduce root growth. In the Rootbox model (Leitner et al., 2010a; Leitner et al., 2010b), the soil medium can moderate root architecture by biasing the direction of root tip growth through a tropism function, as well as modifying the ability of the root tip to extend at its pre-defined distance through a root-elongation modifier. In this study, both functions are the primary means by which the root system's response to urban site impediments and growth attractions are simulated. 


\section{Chapter 3 - Manuscript}

\section{1 - Introduction}

Understanding the growth and architecture of urban tree roots is important for the protection and management of the urban forest (Day et al., 2010). Tree root systems acquire water, nutrient and oxygen resources from the soil, and provide structural support for the tree (Gregory, 2006; Murray, 2011). In urban settings, root growth is often constrained by, and must contend with, a variety of soil conditions, ranging from saturation to drought (Brunner et al., 2015; Day et al., 2010), localized pockets of nutrients and variable access to oxygen (Randrup et al., 2001), compaction (Alberty et al., 1984; Day et al., 2010; Page et al., 2015), and spatial barriers to growth, such as soil volume, debris, and urban infrastructure such as pipes, walls, and pavement (Page et al., 2015; Randrup, et al., 2001). Tree root growth responds to these site heterogeneities and constraints in diverse ways (Brunner et al., 2015), which makes anticipating root system architecture and spatial distribution of roots difficult (Day et al., 2010).

The study of living tree root systems is difficult because most roots are not easily observed without excavation (Day \& Wiseman, 2010; Day et al., 2010). Excavation is often resource intensive, expensive, invasive and destructive (Amato et al., 2008; Lontoc-Roy, et al., 2006). Non-invasive root detection methods exist which can avoid the need for excavation, such as the use of Ground Penetrating Radar (GPR), but detection of roots in urban conditions using this method has proven difficult (Danjon et al., 2008; Stokes et al., 2002) and has been especially challenged by the lack of accurate, objective and automated methods of detection (Guo et al., 2013).

Plant growth modelling approaches offer a potential complement and aid to non-invasive site detection approaches like GPR. Plant growth models can be used to simulate various structural and functional characteristics of a root system (Fourcaud, et al., 2008), and can therefore offer a way to investigate existing or potential tree growing locations within a virtual environment. The use of plant root growth models may also facilitate landscape planning and design by enabling researchers and planners to create scenarios that reflect different assumptions about how root systems may be structurally arranged and function underground (Pagès et al., 2004; Tobin et al., 2007). Additionally, if designed to do so, these models may enable the prediction of the location 
of roots and may anticipate their geometric and topological attributes, which can provide a valuable tool to complement field-observation (Garré et al., 2012).

Root system models / simulations are typically of two kinds: density-based models and architecture models (Gregory, 2006). Density-based models simulate the spatial arrangement of root density, irrespective of morphological characteristics like root branch order, orientation and direction, and spatial arrangement (Kaligiros et al., 2016). These density-based methods can take a variety of approaches. For instance, Heinen et al. (2003) model the growth of a root system as a process of diffusion of root length density. Reddy et al. (2001) simulate root spread as a convective-dispersive process. Dupuy et al., (2005) model the growth of a root system as spatial arrangement of root branching density.

On the other hand, root system architecture models explicitly simulate the morphological characteristics of a root system in three-dimensions (Dunbabin et al., 2013; Fourcaud, et al., 2008; Leitner et al., 2010a; Pagès et al., 2014). The basic output of a root architecture model is an explicit three-dimensional digital representation of the spatial arrangement and location of roots, their individual geometries (e.g. diameter and length), and their topology (relationship to other parts of the root system) (e.g. whether a root is a terminal member, or whether it is an initial basal root). Root architecture models are often computationally expensive because of the spatial complexity of the systems they are modelling. Moreover, these models have been used to simulate root system architecture at different scales, from root hairs (Lenk et al., 2014) to coarser level roots (Mulia et al., 2010; Wagner et al., 2011). More recently, root architecture models have been used in combination with density-based models to reduce computational requirements while also preserving the capacity to model complex root-environment relationships (Dunbabin et al., 2013; Leitner et al., 2010c; Mulia et al., 2010; Pagès et al., 2014).

Root architecture models vary in their focus. Some architecture models are structural, and focus on the creation of three-dimensional geometries (Pagès et al., 2014). Other models explicitly include algorithms for modelling root functions, such as water and nutrient uptake, that require the spatial location of root geometry to be considered. These models are often referred to in the literature as functional-structural models, because they simulate the interaction of geometric as well as specific biological attributes of the plant (Godin et al., 2005). Every root architecture model will at its most basic include routines for simulating a base three-dimensional geometry, 
and in some cases the implementing software will be equipped with the ability to extend the model with customized modules that, for example, will describe root-soil interactions (Leitner et al., 2010a).

While structural and functional-structural architectural models have been applied to both nonwoody and tree species, they have been mainly deployed in contexts that are not site specific (e.g. in hypothetical pots or containers), and which enable the study or testing of plant response to specific growth conditions, such as response to changes in the water content of surrounding soil (Dunbabin, et al., 2013; Leitner et al., 2010b; Mulia et al., 2010). Additionally, most examples of plant root architecture modelling focus on non-woody species; however, more recent research has included tree root systems (Garré et al., 2012). Finally, while several software implementations of these models have been developed, very few have documented best practices, and they are consequently not generally open to wider use or adaptation by practitioners in the field.

This research demonstrates the root architecture model Rootbox, which is an open-source model implemented in MATLAB and authored by David Leitner and Andrea Scnepf, formerly of the University of Vienna's Computational Science Center (Leitner et al., 2010a; University of Vienna, 2017). Rootbox is a general-purpose structural architecture model that can integrate with a three-dimensional soil model. In this study, a set of base growth parameters are selected which reproduce a plausible root system architecture that can adequately reproduce general morphological root characteristics that are common to several tree species. The model is implemented in the context of four hypothetical urban site conditions (scenarios) that are likely to persist through long periods in the tree root system's growth. These conditions are represented by four alterative soil models. The soil models simulate barriers to growth that could be caused by compacted soil or impermeable objects in the soil, and growth biases that could be caused by localized nutrient sources or water infiltration near the soil surface.

The purpose of this research is to assess the capabilities of Rootbox to (1) simulate generalized formal attributes of tree root architecture, and (2) simulate the interaction of tree root architecture with several hypothetical urban growing conditions. While tree root systems have been simulated in other research, and while plant root architecture modelling can have diverse research goals, this research aims to assess the practical nature of Rootbox as a tool that could potentially be 
useful to aid field-investigations conducted by urban forestry pracititioners. If Rootbox can simulate the generalized characteristics of tree root architecture, and hypothetical responses to simulated soil environments, this can then be used as a foundation upon which the design of a more sophisticated, predictive root growth model could be pursued in future research. 


\section{2 - Methods}

The methodological approach taken in this research involves three parts: (1) design of a root architecture simulation in Rootbox by selecting growth parameters that are generative of a plausible root architecture; (2) creation of a set of four soil models that represent hypothetical site conditions that could be found in urban settings; and (3) assessment of the plausibility of the model output to simulate reality as per root architecture descriptions in the literature.

Rootbox's main functions are described first. This is followed by a description of the parameters that are used to create the root system architecture. The method of parameter selection is then described, and followed by an explanation of the design of each of the four soil model scenarios. Finally, the performance of Rootbox output to represent the four scenarios is discussed.

\subsection{1 - Description of Rootbox}

Amongst various models that are available to simulate root architecture, Rootbox (version 6b) was chosen for the current study because it is freely-available, contemporary software that works with a reliable MATLAB code-base. Rootbox is implemented in MATLAB, and uses MATLABbased functions, including functions to create three-dimensional geometries which represent the root system architecture. The MATLAB code-base is easy to understand, is well-documented, and there is a large community of advanced MATLAB users and programmers who can provide technical support. The application authors provide ample in-line script comments (Boswell et al., 2011). The most recent version of the application (University of Vienna, 2017) includes a graphical user interface (GUI) to help users create basic root models that can then be customized as needed through more in-depth programming. The open-source nature of Rootbox makes it potentially accessible to a wide range of researchers and practitioners. Rootbox is provided by its creators as a basic root system architecture model which can be modified or augmented with new modules that describe root-soil interactions - for instance, customized functions can be programmed to describe how the root system should respond to compaction in the soil. Such functions are not explicitly included within Rootbox as originally programmed (Leitner, et al., 2010a). 
Rootbox simulates the growth of a root system over a pre-specified unit of time (usually, in days). This is not the same as the amount of time it takes to run a simulation, which depending on the complexity of the model can be on the order of minutes to a half-hour. Each unit of simulated time (e.g. each day) may be assigned its own rules and parameters, and this can change the rules by which the root system grows through time. In many root systems, the environmental interactions between plant and soil change over time, and operate at different resolutions. To simplify the interpretation of model results and to facilitate comparison between scenarios, parameter settings were kept constant for the entire duration of the simulation.

Model input parameters control the number and growth of individual roots. There are 16 core growth parameters that are set for every root type. These are listed and described in Table 3.1. 
Table 3.1 - The parameters governing root growth in Rootbox, control different aspects of the production rules that create the output architecture. These are listed and described in this table. A '*' on the Parameter Name indicates that this parameter can vary stochastically within a predefined range. A ' $* *$ ' indicates that this parameter (or function) was not used for this study (for reasons that are various but that can include technical difficulties of implementing the function).

\begin{tabular}{|c|c|c|c|}
\hline \# & $\begin{array}{l}\text { Parameter } \\
\text { Name }\end{array}$ & Unit & Description \\
\hline 1 & Elongation rate & $\mathrm{cm}$ & $\begin{array}{l}\text { Extension rate per unit of simulated time (i.e. cm per day). Used to } \\
\text { calculate the overall speed at which an individual root grows } \\
\text { during a simulation. }\end{array}$ \\
\hline 2 & Root radius* & $\mathrm{cm}$ & Radius of the root (does not include its children segments) \\
\hline 3 & Insertion angle* & degrees & $\begin{array}{l}\text { The angle (horizontal axis) at which a successor root will start } \\
\text { growing from its parent. }\end{array}$ \\
\hline 4 & Basal zone * & $\mathrm{cm}$ & $\begin{array}{l}\text { The length of root closest to the branching point where successors } \\
\text { are not permitted to grow. }\end{array}$ \\
\hline 5 & Apical zone $*$ & $\mathrm{~cm}$ & $\begin{array}{l}\text { The length of the root after the basal zone, where successors are } \\
\text { permitted to grow. }\end{array}$ \\
\hline 6 & $\begin{array}{l}\text { Distance } \\
\text { between each } \\
\text { successor* }\end{array}$ & $\mathrm{cm}$ & $\begin{array}{l}\text { The minimum distance the root must grow between successors. } \\
\text { This partially determines the number of successors that will } \\
\text { branch. }\end{array}$ \\
\hline 7 & $\begin{array}{l}\text { Root type of } \\
\text { successor }\end{array}$ & $\mathrm{cm}$ & $\begin{array}{l}\text { This is the root type of the successor that branches from the apical } \\
\text { zone. Successors have their own parameter settings, and this allows } \\
\text { the root system to exhibit different characteristics as it grows. Each } \\
\text { successor type has a probability }\end{array}$ \\
\hline 8 & $\begin{array}{l}\text { Maximum } \\
\text { number of } \\
\text { successors* }\end{array}$ & $\mathrm{cm}$ & The maximum number of successors the root may have. \\
\hline 9 & $\begin{array}{l}\text { Spatial } \\
\text { resolution along } \\
\text { the axis of the } \\
\text { root }\end{array}$ & $\mathrm{cm}$ & The minimum length of each root axis segment. \\
\hline 10 & $\begin{array}{l}\text { Segment } \\
\text { flexibility }\end{array}$ & Unitless & $\begin{array}{l}\text { The ability of each root segment angle to change from the angle of } \\
\text { the previous segment. This allows a root to exhibit random turning } \\
\text { as it grows, which is a desirable quality (Dunbabin et al., 2013) }\end{array}$ \\
\hline 11 & $\begin{array}{l}\text { Tropism } \\
\text { strength }\end{array}$ & Unitless & $\begin{array}{l}\text { The strength of the tropism reaction, which controls the directional } \\
\text { bias of each axis segment when it extends during growth. }\end{array}$ \\
\hline 12 & Tropism type & Unitless & $\begin{array}{l}\text { Tropisms can be either plagio-, gravi-, exo-, or soil grid tropisms. } \\
\text { Tropisms are set per root type. which controls the directional bias } \\
\text { of each axis segment when it extends during growth }\end{array}$ \\
\hline 13 & $\begin{array}{l}\text { Maximum root } \\
\text { lifespan* }\end{array}$ & $\begin{array}{l}\text { Time } \\
\text { unit }\end{array}$ & $\begin{array}{l}\text { The length of time that the root will grow during the simulation. If } \\
\text { the simulation time extends past this point in time, the root and its } \\
\text { children will be deleted from the final output. }\end{array}$ \\
\hline 14 & $\begin{array}{l}\text { Scale } \\
\text { elongation }\end{array}$ & Unitless & $\begin{array}{l}\text { Soil values where a value of }<1 \text { will multiply the elongation rate } \\
\text { of the root (parameter (1)) (e.g. } 0.1 \text { will reduce a } 10 \mathrm{~cm} \text { per unit } \\
\text { time rate by } 90 \% \text { per time unit). }\end{array}$ \\
\hline 15 & $\begin{array}{l}\text { Scale successor } \\
\text { probability }\end{array}$ & Unitless & $\begin{array}{l}\text { Soil values where a value of }<1 \text { will multiply the probability of a } \\
\text { successor (e.g. a } 0.5 \text { will reduce } 10 \text { maximum successors to } 5 \text { ).** }\end{array}$ \\
\hline 16 & $\begin{array}{l}\text { Scale insertion } \\
\text { angle }\end{array}$ & Unitless & $\begin{array}{l}\text { Soil values where a value of }<1 \text { will increase or lower the angle at } \\
\text { which successors are placed on their preceding root } * *\end{array}$ \\
\hline
\end{tabular}


Each set of growth parameters are tailored for each root type. In the present study, 17 root types are specified. The number of types was chosen because this number is the maximum number of types that could be exported for analysis without software errors, which is a project limitation that would likely be solved with some extra programming work. Root types are based on a standard root architecture nomenclature developed by Zobel et al. (2010). There are 4 basic types: Tap, Basal, Shoot-borne, and Laterals up to the $n$th order (or number of successors) (Figure 3.1). In this study, none of the shoot-borne type are used. Small variations in parameter values are used to create a full set of 17 types (Figure 3.2).

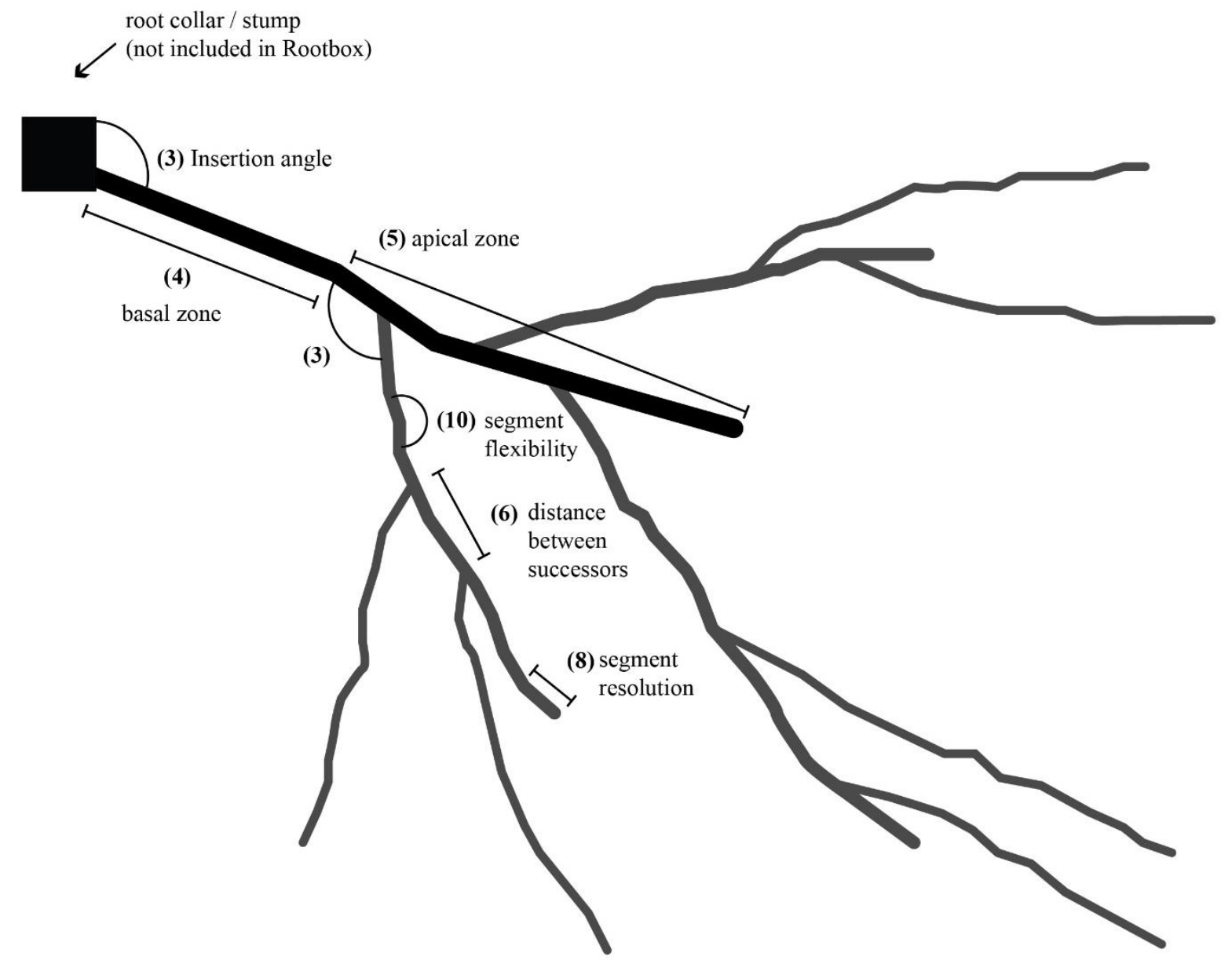

Figure 3.1- A simplified schematic of the various roles that Rootbox's growth parameters play in the production of a root architecture. Each growth parameter is numbered and named (to be read in conjuction with Table 3.1). The initial root, located at the root collar, branches successively into a $2^{\text {nd }}$, and then up to an arbitrary number of orders, which can decrease in diameter / thickness after each branching. In this research, up to 9 orders are used. 


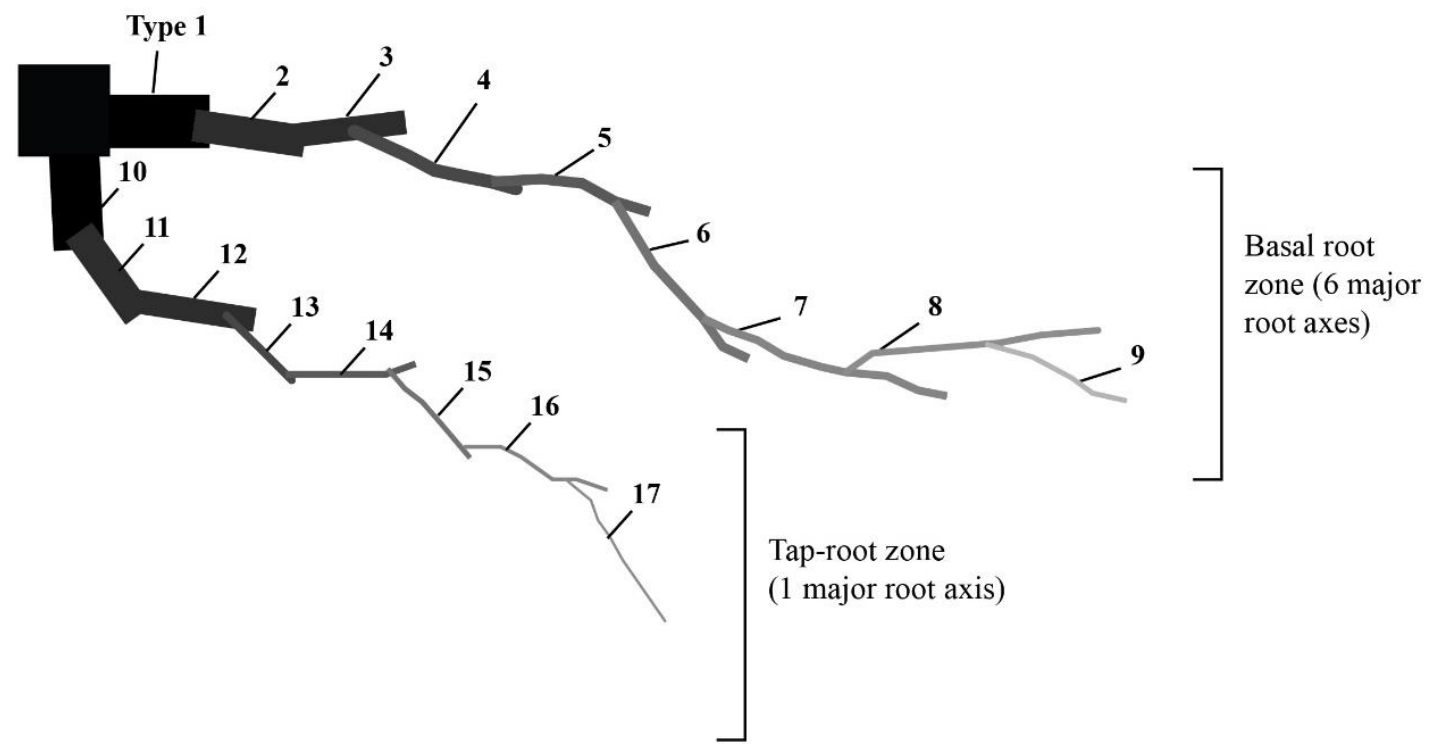

Figure 3.2 - Output root architecture in Rootbox is determined by growth parameters which are set per root type, and root types are topologically organized as a series (or chain) of root successors. This diagram is a description of the number of successor-orders that comprise the final architecture simulations. Up to 9 successors are chosen for the basal root zone, which is the branching structure that grows laterally from the tree base and roughly parallel to the soil surface. Six origin basal roots (type 1) are inserted at 0 degrees from the tree collar. Two successor generations grow out at short distance and reduce rapidly in diameter. A further six generations of successors branch out from each basal root to establish the rest of the root system. A single tap root (type 11) establishes a smaller and more vertically-oriented system to create the "heart" shape as described in Stokes et al. (1996). Roots attached to the tap-root decrease in diameter far more quickly than those attached to basals. 


\subsection{2 - Growth Parameter Selection}

Root growth parameters are selected based on a two-step process: (1) through exploratory runs of the model to determine what parameters create realistic (as per a review of the literature) root system architectures and to determine what is computationally feasible; and (2) refinement of the parameter values based on their ability to create generalized morphological characteristics described in the literature. Danjon et al. (2005) provide several graphical illustrations of a mature pine tree which is used as a guide to creating a "heart" shaped root system, although the precise reproduction of these illustrations is not assessed as a part of determining the realism of the architecture produced by Rootbox. Additionally, illustrations provided by Stokes et al. (2002) and Coutts (1987), photographs of excavated tree systems in Phillips et al. (2015), and digital reconstructions of the root architecture of excavated trees in Nicoll et al. (2006), are used in additional refinement.

Exploratory runs of the model involve setting initial parameter values and then visually assessing whether the resulting architecture is realistic. It is often difficult to gauge how changes in parameter value will influence the model output (Dunbadin, 2007; Lopez et al., 2010; Stava et al., 2011). Through this process, recognizably unrealistic parameter values are ruled out of the final simulations. For example, when parameter 10 (the ability of the root to flex) is set too low, this created unrealistically rigid roots. The meandering morphology of the root, which is a typical morphological trait of most roots found underground (Danjon et al., 2005) is not realistically expressed, given this parameter setting. This "first pass" method is useful in that it narrows the range of model outputs to only plausible outcomes, and is a part of the methodological approach taken by Dunbabin (2007) to assess the importance and sensitivity of parameters of the ROOTMAP architecture model, which is an alternative model to Rootbox, to simulate crop-weed competition.

Parameter refinement involves setting parameter values that reproduce generalized root system characteristics commonly reported in the literature. Refinement of parameters based on how well they conform to similar values in the literature, or how well they produce architectural attributes reported in root system field studies, is a common practice when calibrating architectural models (Collet et al., 2006; Pagès et al., 2004). These generalized characteristics include: 
Zone of rapid taper (ZRT) - Most tree root systems exhibit a rapid change in the average diameter or thickness of roots in the first 1-2 m radius from the base of the tree (Day et al., 2010). Rootbox cannot create a tapering diameter along one root segment during the simulation unless a modification of the model source code is made (Leitner, et al, 2010a). Because a detailed analysis of the source code was not possible for this project, an alternative to root tapering is taken to create a ZRT. Root branching is increased in the first 1-2 m radius by using three generations of short roots that reduce in diameter for each branching. In this way, a chain of three roots are made to "taper" from the $1^{\text {st }}$ to $3^{\text {rd }}$ order root. Each generation is given at most one or two branches, so that over the short length of the roots, at most four different branches for every initial root is created. The rate of taper is established from Danjon et al. (2005), who define a ZRT as the length of the root where the root diameter reduces by $1.25 \%<$ per cm length. To achieve a realistic ZRT, the root radius (parameter 2) of $1^{\text {st }}$ order roots is set to $3 \mathrm{~cm}$ and reduced to $0.3 \mathrm{~cm}$ through to the $7^{\text {th }}$ order roots, and the progression from $1^{\text {st }}$ order to $7^{\text {th }}$ order roots is made to occur over an average of $1.1 \mathrm{~m}$ distance from the base of the tree. The rapid progression through root orders over $1.1 \mathrm{~m}$ is induced by values set for length-controlling parameters. In addition to settings to establish diameter change over distance, diameter values are allowed to fluctuate from root to root, which introduces another varying output of the simulation. The reason this is done is to test the stability of the output ZRT between scenarios; but also to give the outputs more qualitative "realism".

Root system maximal radius based on tree trunk diameter values - The maximal radius distance is a commonly estimated measure in practice and is strongly related to the size of a tree's diameter at breast height (DBH) (Day et al., 2010). There is no consensus within the literature of what the maximum radius of a tree root system is given a value of tree trunk diameter; moreover, this radius will vary dependent on soil volume, soil quality and sub-surface obstacles. Day et al. (2010) indicate that there is a strong relationship between DBH and tree root system radius, and that the relationship can be quantified at a ratio of 1:38 for trees up to $20 \mathrm{~cm}$ $\mathrm{DBH}$. For a tree of $20 \mathrm{~cm} \mathrm{DBH}$, root system radius would be up to approximately $7.5 \mathrm{~m}$. For all hypothetical scenarios, it is assumed that the maximum root system radius is approximately $8 \mathrm{~m}$ (and that the hypothetical tree has a DBH of between 20 and $25 \mathrm{~cm}$ ). This value is not itself an input parameter to the model. Values for root length-controlling parameters are set to generate an overall horizontal length of through the progression of highest to lowest-order roots. 
Root system shape - Root insertion angles (parameter 3) and segment flexibility (parameter 10), described in Table 3.1, are set per how they form growth patterns in profile. Parameters are set by referencing generalized vertical growth patterns described in Stokes et al. (1996). These authors describe three types of root system shapes: (1) plate systems, which are characterized by predominantly horizontal lateral growth with minimal taper and occasional vertical "sinker" roots; (2) tap root systems, where one dominant vertically oriented root occurs under the base of the tree and is supported by a set of smaller horizontally-oriented roots (roughly parallel to soil surface); and (3) heart systems, where both horizontal and vertically oriented roots develop around, and where a high degree of branching occurs in close proximity to, the base of the tree. Plate systems tend to occur in gymnosperms (i.e. non-fruiting trees like pine and spruce) and heart systems tend to occur in angiosperms (i.e. fruiting trees, like oak and maple). These three types of root shape are highly generalized characteristics derived from field observations and are associated with the ability of the root system to provide structural support to the tree (Coutts, 1987). Stokes et al. (1996) do not propose a threshold for when a root system is considered as any of the three types, and some root forms reported in Danjon et. al. (2005) seem to be combinations of each type. While establishing a general shape of the root system is subjective and challenging, for the present study the branching angles of first order roots (i.e. root types 1 , 2, and 3) are set to ensure that they initiate at angles that bias their direction of growth laterally, emanating from the tree base. The insertion angle of a single tap-root (i.e. root type 10) is set at 90 degrees to allow it and its successors to "fill" out the area underneath the base of the tree. A technical limitation of Rootbox is that it does not allow for the the initial horizontal direction of root initiation to be specified in advance, and therefore the angles of high-order root initiation from the tree base are determined at random and render root growth direction hard to control in the horizontal plane.

ZRT, total radial extent, and root system shape are highly generalized characteristics, but are also simple enough to use as guides for setting realistic parameters. The generalization is an advantage because it avoids the methodological problem of calibrating the model to match sitespecific measurements, which may not be representative of common architectural characteristics. The generality of the characteristics is advantageous also because it enables species-agnostic 
characteristics to be built into the simulation, which may allow results to be relevant to a variety of site conditions. Estimation of formal parameters from the literature is not meant to reproduce a site-specific root system (Dunbabin, et al., 2013) and, therefore, the model instead aims to implement realistic production rules that include variability. As discussed in the Discussion section, a potential avenue of future research could focus on customizing Rootbox to conform its output to match site-specific measurements.

\subsection{3 - Hypothetical Scenarios}

Four hypothetical scenarios are developed to capture different and plausible urban site soil conditions. For the first scenario, a "base" soil environment is developed that promotes growth in the upper $0.5 \mathrm{~m}$ of soil and laterally outward, quasi-parallel to the soil surface, from the tree base toward the periphery of the site, and discourages root growth below this plane. For each scenario, this basic soil environment is altered to introduce a growth bias to direct or restrict root growth in specific locations. Additionally, each scenario is iterated five times to account for stochastic variation in the results. Growth parameters are kept constant throughout each scenario, excepting root diameter (as noted above).

One unique soil environment grid is created to describe each of the four hypothetical scenarios. The soil environment grids are created using a four-dimensional matrix grid $(\mathrm{x}, \mathrm{y}, \mathrm{z}, \mathrm{C})$, where $\mathrm{x}$, $\mathrm{y}$, and $\mathrm{z}$ are the three-dimensional spatial coordinates and $\mathrm{C}$ is a floating value at the spatial coordinate of the grid that encodes a soil value. The grid itself is created using the MATLAB meshgrid function that can be modified to load values from a comma delimited value (.csv) file that is created separately in Microsoft Office Excel or from internal grid-creation functions within MATLAB. The values encoded in each soil grid do not directly represent real-world measurements (such as, for example, soil bulk-density, soil compaction, soil oxygen availability).

There are two types of functions that may be triggered by a soil grid: (1) a tropism response, which modulates the growth direction of the root; and (2) an elongation response, which slows or speeds the rate at which a root will extend. Tropism is commonly observed in many plants, but the causes of tropism are diverse (Firn et al.,1980; Leitner, et al., 2010a) and it is not necessarily 
clear how to account for it within a soil model (i.e. how to translate soil characteristics that influence plant growth into tropism modifying values). Soil grid values that are below 1 (i.e. between 0 and 0.99) can trigger an elongation response. The elongation response is goverened by a function that decreases the rate of elongation by the value encountered at that location in the soil grid. For instance, a value of 0.5 will slow root extension by $50 \%$ at that location. This can can be used, for example, to model the impact of root growth retarding influences such as high soil bulk density, compacted soil or low soil oxygen concentration. Creating a soil environment and setting the response functions offers a way to control the shape and spatial distribution of the resulting root system. However, the directional tropism function does not result in individual roots following a pre-defined directional pathway, nor do roots grow rigidly towards a directional influence. Rather, the tropism function increases the chances of any particular root tip to orient in the direction of influence. In this research, soil grid values vary between each scenario, but only enough to create localized idiosyncrasies, such as the setting of very low soil grid values for compacted areas under a sidewalk.

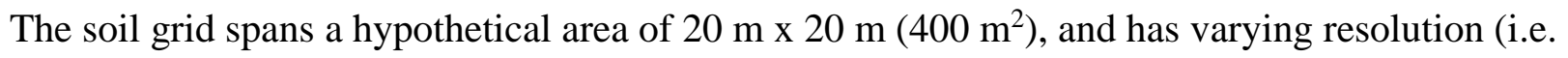
there are more than 400 grid points). In the first $4 \mathrm{~m}$ distance, grids occur at every $0.25 \mathrm{~m}$, and after $4 \mathrm{~m}$, every $1 \mathrm{~m}$. The higher resolution of the grid nearer the origin is due mainly to the need to keep computation resources light; while it is desirable to provide a consistent and small resolution, simulations run too slowly when the entire grid is at $0.25 \mathrm{~m}$ intervals.

\section{Scenario 1 - Base Grid}

Scenario 1 represents a soil condition without growth impedance or any varying directional bias. An open field condition, which may be encountered in larger urban parks, is an example of a site where these conditions may be found. Soil grid values are made to cause the roots to tend outwards and to explore the upper $0.5 \mathrm{~m}$ of the soil profile (see Figures 3.3 and 3.4).

This bias was created to ensure that the root system exploits soil layers closest to the surface and that they exploit distant soil in a radial pattern. The preference of root systems to exploit shallower soil layers and to avoid deeper layers is a common characteristic of most tree root systems (Coutts, 1987; Couttset al., 1991; Crow, 2005; Day et al., 2010; Day \& Harris, 2008), although the biological reasons for this growth predilection in shallower soils are diverse. 


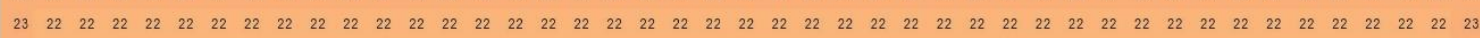
$\begin{array}{lllllllllllllllllllllllllllllllllllllllllllllllllll}8 & 23 & 22 & 21 & 21 & 21 & 21 & 21 & 21 & 21 & 21 & 21 & 21 & 21 & 21 & 21 & 21 & 21 & 21 & 21 & 21 & 21 & 21 & 21 & 21 & 21 & 21 & 21 & 21 & 21 & 21 & 21 & 21 & 21 & 21 & 21 & 21 & 21 & 21 & 21 & 21 & 21 & 21 & 21 & 22 & 23\end{array}$

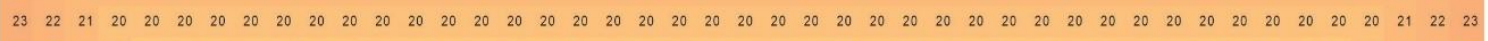

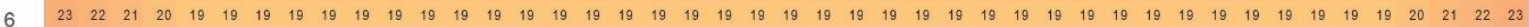
$\begin{array}{lllllllllllllllllllllllllllllllllllllllllllllllllllll}23 & 22 & 21 & 20 & 19 & 18 & 18 & 18 & 18 & 18 & 18 & 18 & 18 & 18 & 18 & 18 & 18 & 18 & 18 & 18 & 18 & 18 & 18 & 18 & 18 & 18 & 18 & 18 & 18 & 18 & 18 & 18 & 18 & 18 & 18 & 18 & 18 & 18 & 18 & 18 & 18 & 20 & 21 & 22 & 23\end{array}$

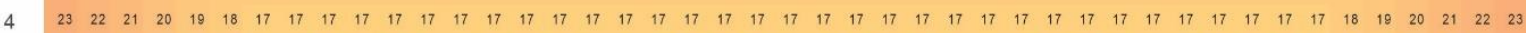

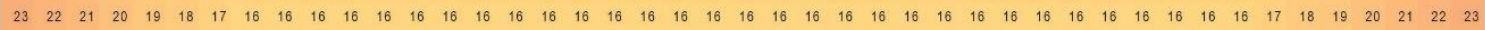
$\begin{array}{llllllllllllllllllllllllllllllllllllllllllllllllllllllllll}23 & 22 & 21 & 20 & 19 & 18 & 17 & 16 & 15 & 15 & 15 & 15 & 15 & 15 & 15 & 15 & 15 & 15 & 15 & 15 & 15 & 15 & 15 & 15 & 15 & 15 & 15 & 15 & 15 & 15 & 15 & 15 & 15 & 15 & 15 & 15 & 15 & 16 & 17 & 18 & 19 & 20 & 21 & 22 & 23\end{array}$

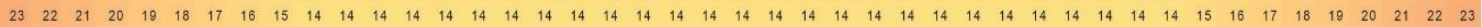
$\begin{array}{llllllllllllllllllllllllllllllllllllllllllllllllll}23 & 22 & 21 & 20 & 19 & 18 & 17 & 16 & 15 & 14 & 13 & 13 & 13 & 13 & 13 & 13 & 13 & 13 & 13 & 13 & 13 & 13 & 13 & 13 & 13 & 13 & 13 & 13 & 13 & 13 & 13 & 13 & 13 & 13 & 13 & 14 & 15 & 16 & 17 & 18 & 19 & 20 & 21 & 22 & 23\end{array}$

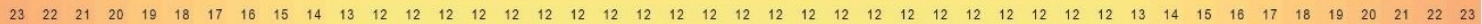
$\begin{array}{llllllllllllllllllllllllllllllllllllllllllllllllll}23 & 22 & 21 & 20 & 19 & 18 & 17 & 16 & 15 & 14 & 13 & 12 & 11 & 11 & 11 & 11 & 11 & 11 & 11 & 11 & 11 & 11 & 11 & 11 & 11 & 11 & 11 & 11 & 11 & 11 & 11 & 11 & 11 & 12 & 13 & 14 & 15 & 16 & 17 & 18 & 19 & 20 & 21 & 22 & 23\end{array}$

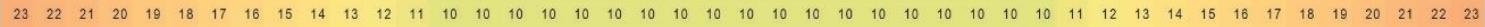

$\begin{array}{llllllllllllllllllllllllllllllllllllllllllllllllllllllllll}2 & 23 & 22 & 21 & 20 & 19 & 18 & 17 & 16 & 15 & 14 & 13 & 12 & 11 & 10 & 9 & 9 & 9 & 9 & 9 & 9 & 9 & 9 & 9 & 9 & 9 & 9 & 9 & 9 & 9 & 9 & 9 & 10 & 11 & 12 & 13 & 14 & 15 & 16 & 17 & 18 & 19 & 20 & 21 & 22 & 23\end{array}$ $\begin{array}{lllllllllllllllllllllllllllllllllllllllllllllllllllllllllll}23 & 22 & 21 & 20 & 19 & 18 & 17 & 16 & 15 & 14 & 13 & 12 & 11 & 10 & 9 & 8 & 8 & 8 & 8 & 8 & 8 & 8 & 8 & 8 & 8 & 8 & 8 & 8 & 8 & 8 & 9 & 10 & 11 & 12 & 13 & 14 & 15 & 16 & 17 & 18 & 19 & 20 & 21 & 22 & 23\end{array}$ $\begin{array}{lllllllllllllllllllllllllllllllllllllllllllllllllllllllllll}23 & 22 & 21 & 20 & 19 & 18 & 17 & 16 & 15 & 14 & 13 & 12 & 11 & 10 & 9 & 8 & 7 & 7 & 7 & 7 & 7 & 7 & 7 & 7 & 7 & 7 & 7 & 7 & 7 & 8 & 9 & 10 & 11 & 12 & 13 & 14 & 15 & 16 & 17 & 18 & 18 & 20 & 21 & 22 & 23\end{array}$ $\begin{array}{llllllllllllllllllllllllllllllllllllllllllllllllllllllllllll}23 & 22 & 21 & 20 & 19 & 18 & 17 & 16 & 15 & 14 & 13 & 12 & 11 & 10 & 9 & 8 & 7 & 6 & 6 & 6 & 6 & 6 & 6 & 6 & 6 & 6 & 6 & 6 & 7 & 8 & 9 & 10 & 11 & 12 & 13 & 14 & 15 & 16 & 17 & 18 & 19 & 20 & 21 & 22 & 23\end{array}$ $\begin{array}{lllllllllllllllllllllllllllllllllllllllllllllllllllllll}23 & 22 & 21 & 20 & 19 & 18 & 17 & 16 & 15 & 14 & 13 & 12 & 11 & 10 & 9 & 8 & 7 & 6 & 5 & 5 & 5 & 5 & 5 & 5 & 5 & 5 & 5 & 6 & 7 & 8 & 9 & 10 & 11 & 12 & 13 & 14 & 15 & 16 & 17 & 18 & 19 & 20 & 21 & 22 & 23\end{array}$ $\begin{array}{llllllllllllllllllllllllllllllllllllllllllllllllllllllll}23 & 22 & 21 & 20 & 19 & 18 & 17 & 16 & 15 & 14 & 13 & 12 & 11 & 10 & 9 & 8 & 7 & 6 & 5 & 4 & 4 & 4 & 4 & 4 & 4 & 4 & 5 & 6 & 7 & 8 & 9 & 10 & 11 & 12 & 13 & 14 & 15 & 16 & 17 & 18 & 19 & 20 & 21 & 22 & 23\end{array}$ $\begin{array}{lllllllllllllllllllllllllllllllllllllllllllllllllllllllllll}23 & 22 & 21 & 20 & 19 & 18 & 17 & 16 & 15 & 14 & 13 & 12 & 11 & 10 & 9 & 8 & 7 & 6 & 5 & 4 & 3 & 3 & 3 & 3 & 3 & 4 & 5 & 6 & 7 & 8 & 9 & 10 & 11 & 12 & 13 & 14 & 15 & 16 & 17 & 18 & 18 & 20 & 21 & 22 & 23\end{array}$ $\begin{array}{llllllllllllllllllllllllllllllllllllllllllllllllllll}23 & 22 & 21 & 20 & 19 & 18 & 17 & 16 & 15 & 14 & 13 & 12 & 11 & 10 & 9 & 8 & 7 & 6 & 5 & 4 & 3 & 2 & 2 & 2 & 3 & 4 & 5 & 6 & 7 & 8 & 9 & 10 & 11 & 12 & 13 & 14 & 15 & 16 & 17 & 18 & 19 & 20 & 21 & 22 & 23\end{array}$ \begin{tabular}{lllllllllllllllllllllll|lllllllllllllllllllllllllllllll}
23 & 22 & 21 & 20 & 19 & 18 & 17 & 16 & 15 & 14 & 13 & 12 & 11 & 10 & 9 & 8 & 7 & 6 & 5 & 4 & 3 & 2 & 1 & 2 & 3 & 4 & 5 & 6 & 7 & 8 & 9 & 10 & 11 & 12 & 13 & 14 & 15 & 16 & 17 & 18 & 19 & 20 & 21 & 22 & 23
\end{tabular} $\begin{array}{llllllllllllllllllllllllllllllllllllllllllllllllllllll}23 & 22 & 21 & 20 & 19 & 18 & 17 & 16 & 15 & 14 & 13 & 12 & 11 & 10 & 9 & 8 & 7 & 6 & 5 & 4 & 3 & 2 & 2 & 2 & 3 & 4 & 5 & 6 & 7 & 8 & 9 & 10 & 11 & 12 & 13 & 14 & 15 & 16 & 17 & 18 & 18 & 20 & 21 & 22 & 23\end{array}$ $\begin{array}{llllllllllllllllllllllllllllllllllllllllllllllllllllll}23 & 22 & 21 & 20 & 19 & 18 & 17 & 16 & 15 & 14 & 13 & 12 & 11 & 10 & 9 & 8 & 7 & 6 & 5 & 4 & 3 & 3 & 3 & 3 & 3 & 4 & 5 & 6 & 7 & 8 & 9 & 10 & 11 & 12 & 13 & 14 & 15 & 16 & 17 & 18 & 19 & 20 & 21 & 22 & 23\end{array}$ $\begin{array}{lllllllllllllllllllllllllllllllllllllllllllllllllllllllll}23 & 22 & 21 & 20 & 19 & 18 & 17 & 16 & 15 & 14 & 13 & 12 & 11 & 10 & 9 & 8 & 7 & 6 & 5 & 4 & 4 & 4 & 4 & 4 & 4 & 4 & 5 & 6 & 7 & 8 & 9 & 10 & 11 & 12 & 13 & 14 & 15 & 16 & 17 & 18 & 19 & 20 & 21 & 22 & 23\end{array}$ $\begin{array}{llllllllllllllllllllllllllllllllllllllllllllllllllllllllll}23 & 22 & 21 & 20 & 19 & 18 & 17 & 16 & 15 & 14 & 13 & 12 & 11 & 10 & 9 & 8 & 7 & 6 & 5 & 5 & 5 & 5 & 5 & 5 & 5 & 5 & 5 & 6 & 7 & 8 & 9 & 10 & 11 & 12 & 13 & 14 & 15 & 16 & 17 & 18 & 18 & 20 & 21 & 22 & 23\end{array}$ $\begin{array}{llllllllllllllllllllllllllllllllllllllllllllllllllllllllll}23 & 22 & 21 & 20 & 19 & 18 & 17 & 16 & 15 & 14 & 13 & 12 & 11 & 10 & 9 & 8 & 7 & 6 & 6 & 6 & 6 & 6 & 6 & 6 & 6 & 6 & 6 & 6 & 7 & 8 & 9 & 10 & 11 & 12 & 13 & 14 & 15 & 16 & 17 & 18 & 18 & 20 & 21 & 22 & 23\end{array}$ $\begin{array}{lllllllllllllllllllllllllllllllllllllllllllllllllllllllllll}23 & 22 & 21 & 20 & 19 & 18 & 17 & 16 & 15 & 14 & 13 & 12 & 11 & 10 & 9 & 8 & 7 & 7 & 7 & 7 & 7 & 7 & 7 & 7 & 7 & 7 & 7 & 7 & 7 & 8 & 9 & 10 & 11 & 12 & 13 & 14 & 15 & 16 & 17 & 18 & 19 & 20 & 21 & 22 & 23\end{array}$ $\begin{array}{lllllllllllllllllllllllllllllllllllllllllllllllllllllllllll}23 & 22 & 21 & 20 & 19 & 18 & 17 & 16 & 15 & 14 & 13 & 12 & 11 & 10 & 9 & 8 & 8 & 8 & 8 & 8 & 8 & 8 & 8 & 8 & 8 & 8 & 8 & 8 & 8 & 8 & 9 & 10 & 11 & 12 & 13 & 14 & 15 & 16 & 17 & 18 & 18 & 20 & 21 & 22 & 23\end{array}$ $\begin{array}{llllllllllllllllllllllllllllllllllllllllllllllllllllllllll}23 & 22 & 21 & 20 & 19 & 18 & 17 & 16 & 15 & 14 & 13 & 12 & 11 & 10 & 9 & 9 & 9 & 9 & 9 & 9 & 9 & 9 & 9 & 9 & 9 & 9 & 9 & 9 & 9 & 9 & 9 & 10 & 11 & 12 & 13 & 14 & 15 & 16 & 17 & 18 & 18 & 20 & 21 & 22 & 23\end{array}$

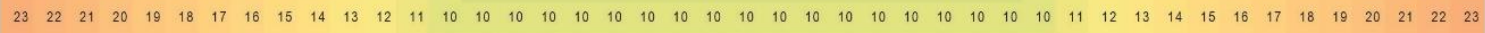
$\begin{array}{lllllllllllllllllllllllllllllllllllllllllllllllll}23 & 22 & 21 & 20 & 19 & 18 & 17 & 16 & 15 & 14 & 13 & 12 & 11 & 11 & 11 & 11 & 11 & 11 & 11 & 11 & 11 & 11 & 11 & 11 & 11 & 11 & 11 & 11 & 11 & 11 & 11 & 11 & 11 & 12 & 13 & 14 & 15 & 16 & 17 & 18 & 19 & 20 & 21 & 22 & 23\end{array}$

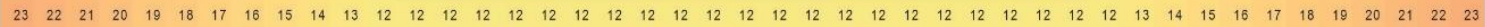
$\begin{array}{llllllllllllllllllllllllllllllllllllllllllllllllll}23 & 22 & 21 & 20 & 19 & 18 & 17 & 16 & 15 & 14 & 13 & 13 & 13 & 13 & 13 & 13 & 13 & 13 & 13 & 13 & 13 & 13 & 13 & 13 & 13 & 13 & 13 & 13 & 13 & 13 & 13 & 13 & 13 & 13 & 13 & 14 & 15 & 16 & 17 & 18 & 19 & 20 & 21 & 22 & 23\end{array}$

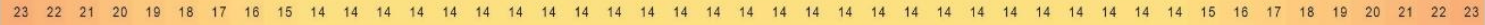
$\begin{array}{lllllllllllllllllllllllllllllllllllllllllllllllllllllll}23 & 22 & 21 & 20 & 19 & 18 & 17 & 16 & 15 & 15 & 15 & 15 & 15 & 15 & 15 & 15 & 15 & 15 & 15 & 15 & 15 & 15 & 15 & 15 & 15 & 15 & 15 & 15 & 15 & 15 & 15 & 15 & 15 & 15 & 15 & 15 & 15 & 16 & 17 & 18 & 19 & 20 & 21 & 22 & 23\end{array}$

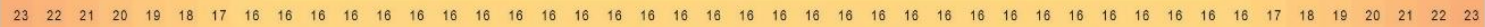

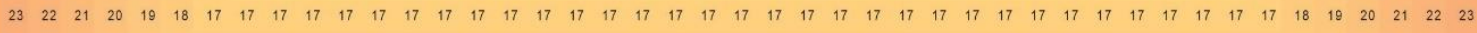
$\begin{array}{llllllllllllllllllllllllllllllllllllllllllllllllllllllllll}23 & 22 & 21 & 20 & 19 & 18 & 18 & 18 & 18 & 18 & 18 & 18 & 18 & 18 & 18 & 18 & 18 & 18 & 18 & 18 & 18 & 18 & 18 & 18 & 18 & 18 & 18 & 18 & 18 & 18 & 18 & 18 & 18 & 18 & 18 & 18 & 18 & 18 & 18 & 18 & 19 & 20 & 21 & 22 & 23\end{array}$ $\begin{array}{lllllllllllllllllllllllllllllllllllllllllllllllllllll}23 & 22 & 21 & 20 & 19 & 19 & 19 & 19 & 19 & 19 & 19 & 19 & 19 & 19 & 19 & 19 & 19 & 19 & 19 & 19 & 19 & 19 & 19 & 19 & 19 & 19 & 19 & 19 & 19 & 19 & 19 & 19 & 19 & 19 & 19 & 19 & 19 & 19 & 19 & 19 & 19 & 20 & 21 & 22 & 23\end{array}$

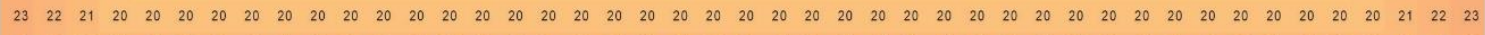

$\begin{array}{lllllcllll}-10 & -8 & -6 & -4 & -2 & 0 & 2 & 23 & 10\end{array}$

Figure 3.3 - Surface view (ortho-graphic top-down) of the base grid used with Scenario 1. The grid ranges in values from 1 (dark green) to 23 (orange) at the edges of the grid. Grid values are shown on top of the cell colours. The grid spans $20 \mathrm{~m}$ x $20 \mathrm{~m}$, but its resolution varies. Grid values increment from smaller values (at the origin point in the center, and indicated with a small black border) to larger values at the periphery because Rootbox's tropism function requires that the directional bias should be represented by increasing grid values in the direction of influence. 


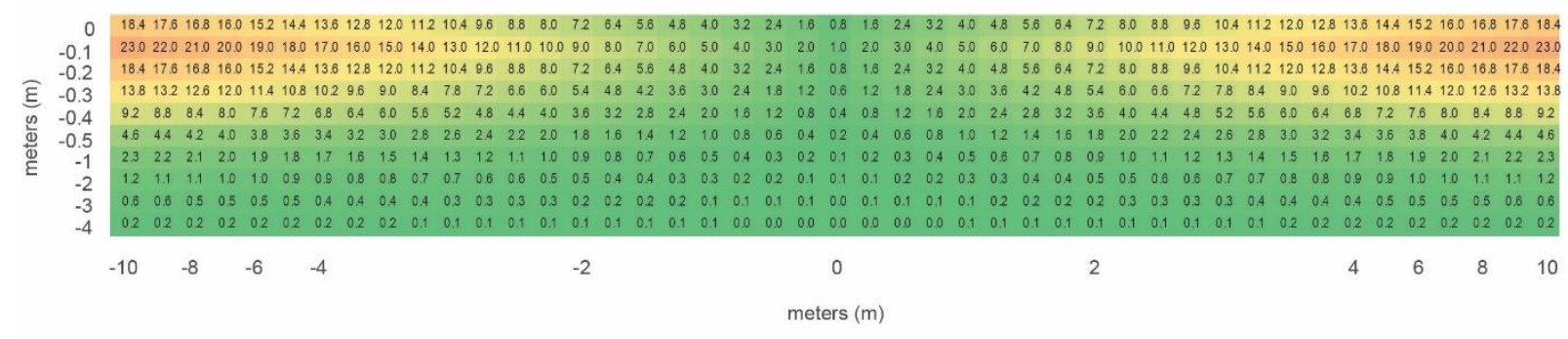

Figure 3.4 - Profile view (i.e. as a slice through the grid and seen from the side) of the base grid of Scenario 1. Dark green values are values that approach zero, and these values limit extension of root growth below $1 \mathrm{~m}$ in depth. The location at $(0,0)$ contains low values (i.e. one) that increases to 23 at the edges (i.e. at $10 \mathrm{~m}$ horizontal distance and down to to $0.5 \mathrm{~m}$ depth). Grid values are highest at $0.1 \mathrm{~m}$ below the surface, to make sure that roots do not collect just below the surface line. 


\section{Scenario 2 - Nutrient Source or Leaky Pipe}

For Scenario 2, a hypothetical nutrient or water source is included within the soil grid. This scenario may describe urban conditions where there is a sewage or water pipe leak under ground (Randrup et al., 2001), and through which a tropism influences the roots to direct themselves towards the nutrient or water source. The bias does not act upon the entire root system and cannot be detected by root tips at all parts of the soil environment. If it did, then the entire root system would be expected to respond to the tropism effect, and each root would gradually change its directional growth towards the point of attraction. However, in real site growth conditions, it is more likely that roots within a given proximity to the attraction point will begin moving towards it. Here, grid values remain the same as in Scenario 1, but grid values increase in a localized area at approximately $2.5 \mathrm{~m}$ from the base of the tree and $0.3 \mathrm{~m}$ below the surface (see Figures 3.5 and 3.6). Grid values for the directional bias are established by multiplying by three grid points within a buffer of $0.75 \mathrm{~m}$ on either side, $0.2 \mathrm{~m}$ above, and $1.2 \mathrm{~m}$ below, the "leak" point. The internal location (grid cells) describing the water or nutrient conduit is given a soil value of zero because this represents an obstacle to root growth. 


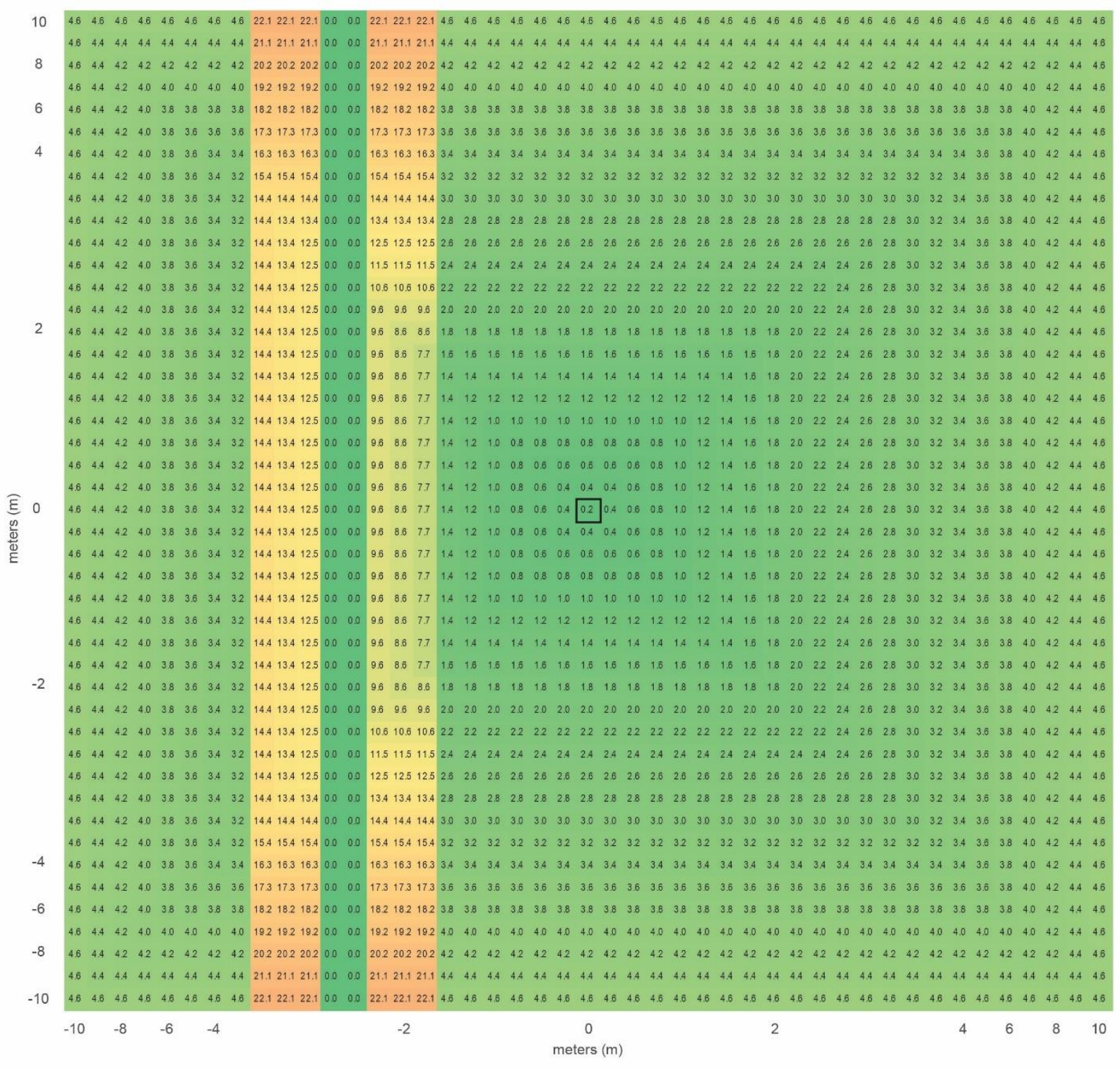

Figure 3.5 - Surface view of the soil grid of Scenario 2. The surface view shows values as they would appear at $0.5 \mathrm{~m}$ depth. The hypothetical nutrient or water source is represented by high grid values along a conduit that runs across the site. The conduit is located approximately $2.5 \mathrm{~m}$ away from the base of the tree, and between 0.3 and $0.4 \mathrm{~m}$ below the surface. However, the actual zone of influence, where roots start to direct themselves towards the conduit, is $0.75 \mathrm{~m}$ on either side, and $1.2 \mathrm{~m}$ below the conduit. 


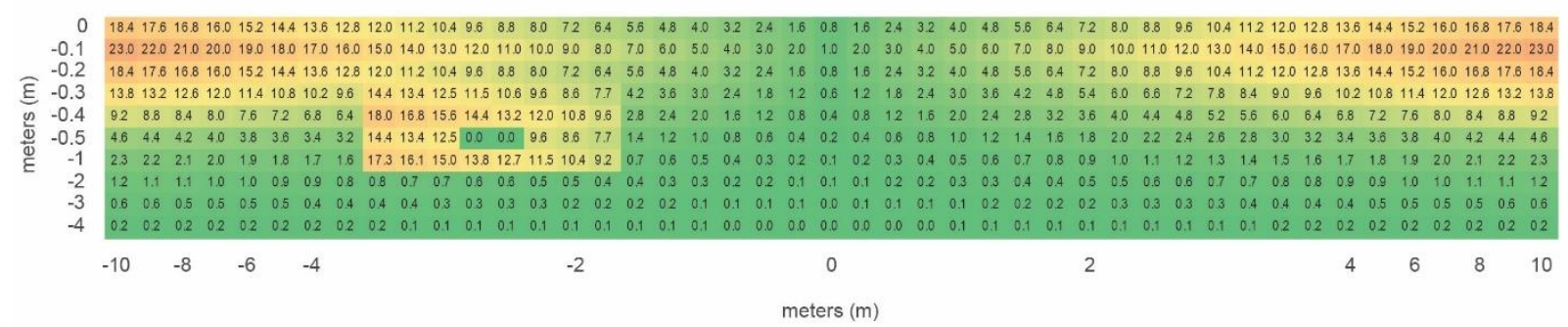

Figure 3.6 - Profile view of the soil grid used in Scenario 2. The hypothetical nutrient or water source is represented by high grid values along a conduit that runs across the site. The conduit is located approximately $2.5 \mathrm{~m}$ away from the base of the tree, and between 0.3 and $0.4 \mathrm{~m}$ below the surface. However, the actual zone of influence, where roots start to direct themselves towards the conduit, is $0.75 \mathrm{~m}$ on either side, and $1.2 \mathrm{~m}$ below the conduit. The grid is identical to the base grid, except the higher values that represent the nutrient or water resource is located at a 0.3$0.4 \mathrm{~m}$ depth. 


\section{Scenario 3 - Sidewalk (Physical Barrier) Underlaid with Compacted Soil}

In Scenario 3, a sidewalk is located at $1 \mathrm{~m}$ away from the base of the tree, and below it is compacted soil to a depth of $0.5 \mathrm{~m}$. Soil grid values reflecting sidewalk compaction are represented by low values, which trigger both the tropism and elongation functions, forcing the root to change direction or stop growing underneath the sidewalk (see Figures 3.7 and 3.8). The compaction in this case can either be modelled as a hard barrier, where root growth is forced to stop completely upon encountering low grid values (e.g. when a concrete sidewalk is encountered), or it can be modelled to make root growth more difficult by making the values under the sidewalk between zero and one (e.g. 0.5; compacted soil). In the former case, roots will fail to extend if they find themselves entering the volume under the sidewalk. At values of zero, extension of a root stops immediately. In the latter case, roots will tend to seek out unimpeded soil if they find themselves growing in the compacted area or, they will grow for a short distance before terminating. In this scenario, depths between down to $0.1 \mathrm{~m}$ beneath the surface represent the hard material, such as concrete, used to create a sidewalk, and so grid values at this depth are a hard barrier that will not permit extension. In this case, the barrier is a sidewalk, but could stand for any object that creates a barrier to growth, such as a wall, or a piece of underground infrastructure. 


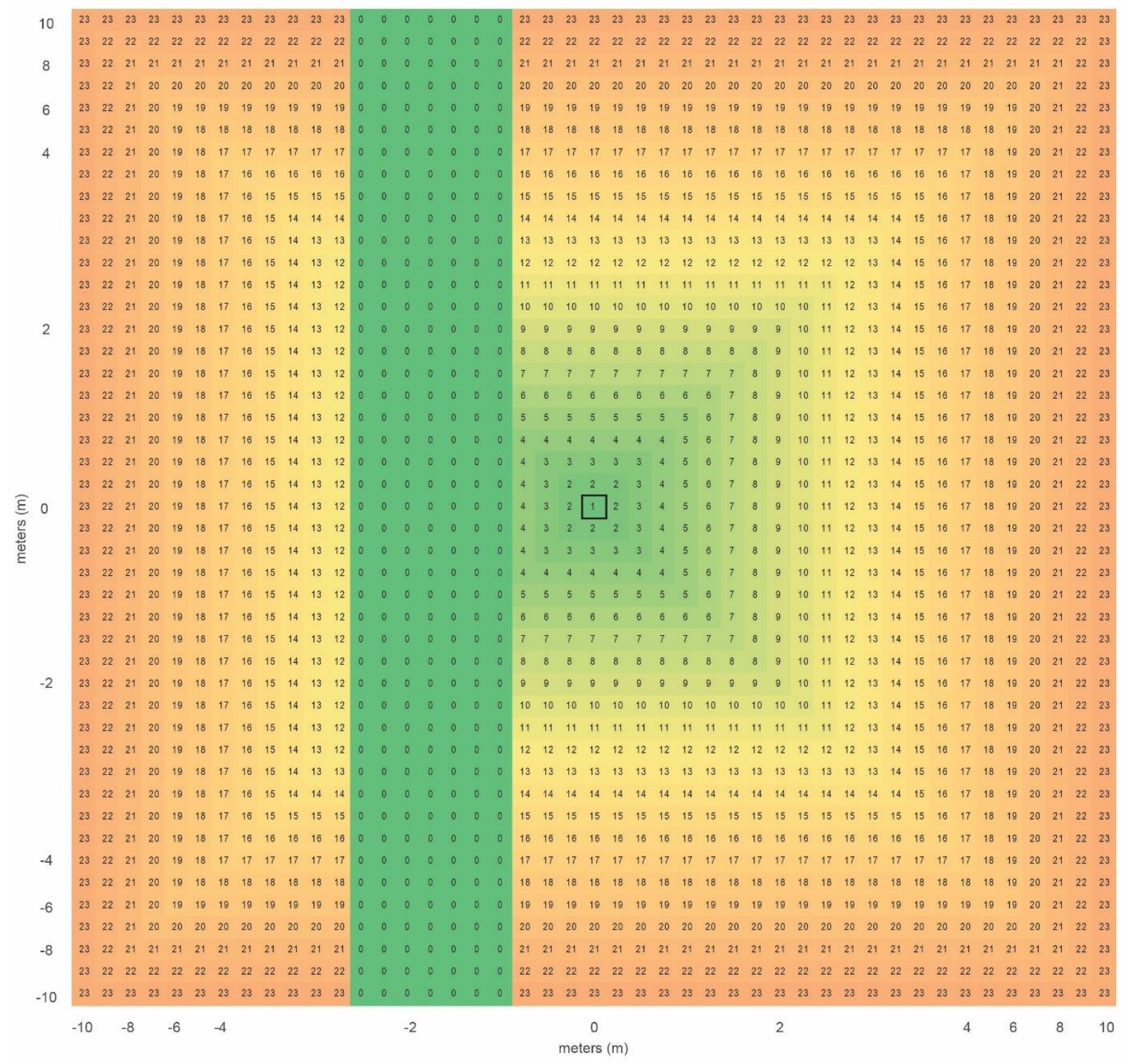

Figure 3.7 - Surface view of the soil grid of Scenario 3. Like Scenario 2, the grid is identical to the base grid of Scenario 1, except for the very low values representing the location of the sidewalk, represented here as dark green. The sidewalk is located approx. $1 \mathrm{~m}$ distance from the base of the tree (indicated by a black square), is $1.5 \mathrm{~m}$ wide along the entire site, and creates a volume of low grid values up to $0.5 \mathrm{~m}$ deep. 


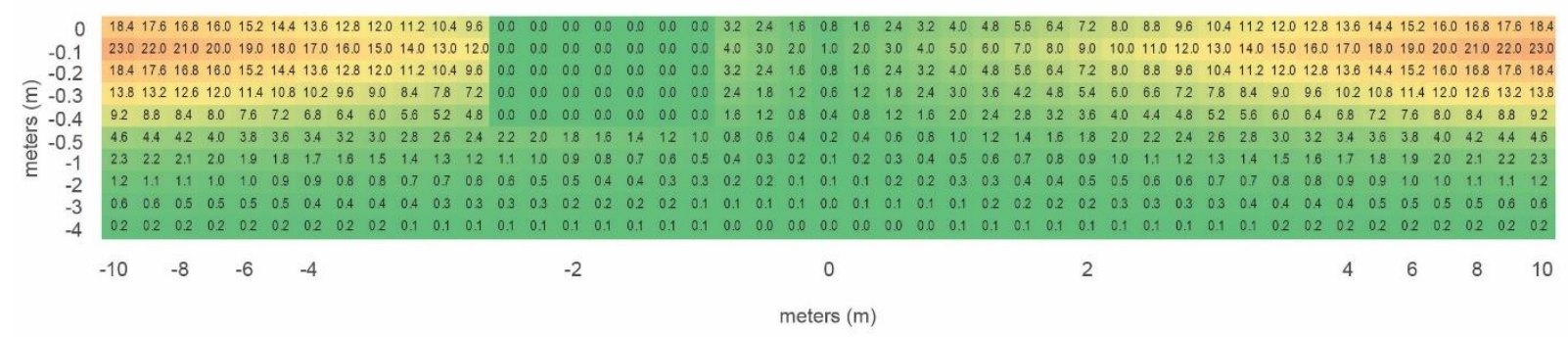

Figure 3.8 - Profile view of the soil grid of Scenario 3. Like Scenario 2, the grid is identical to the base grid of Scenario 1, except for the very low values at the location of the sidewalk, which is graphically represented as a dark green and grid values of 0 at the top of the profile. The sidewalk is located approx. $1 \mathrm{~m}$ distance from the base of the tree, is $1.5 \mathrm{~m}$ wide along the entire site, and creates a volume of low grid values up to $0.5 \mathrm{~m}$ deep. 


\section{Scenario 4 - Weighting the Basic Grid Based on In-Situ Knowledge of Root Location}

In Scenario 4, a customized grid is created to represent areas where a hypothetical on-site field investigation has revealed the presence of roots and/or a substantial amount of root biomass. Details of the root detection approach are not relevant to the scenario creation and are, therefore, not discussed here.

The base grid is weighted to favour these areas of known root presence. The weighting scheme is encoded into a weighting grid, which is multiplied against the base grid to create a tropism response that directs root growth to these locations. A multiplication of base grid values to create tropism bias is used in Scenario 2, but in that scenario the bias is localized within a limited zone of influence. Scenario 4 takes a different approach and weights the entirety of the base grid to ensure that the effect of the bias is present in all locations and signals all active root tips.

Two locations are chosen to represent areas of the highest directional bias (see Figures 3.9 and 3.10). These are located at the three-dimensional spatial co-ordinates (in cm): -500 (x1); -325 (y1); -50 (z1) and $300(\mathrm{x} 2) ; 375(\mathrm{y} 2) ;-40(\mathrm{z} 2)$. At both locations, grid values are weighted by 2 (i.e. plus $100 \%$ of the original value), and immediately adjacent values are weighted by 1.956 (i.e. plus $95.6 \%$ of its original value).

Weighting all values of the base grid has the effect of preserving the overall distribution of high/low values of the base grid (i.e. higher values near the surface and towards the periphery), while at the same time it amplifies these values. 


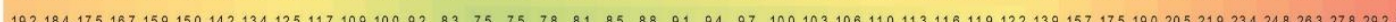

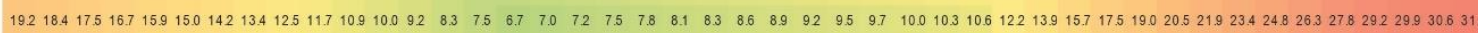

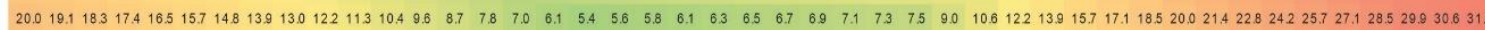

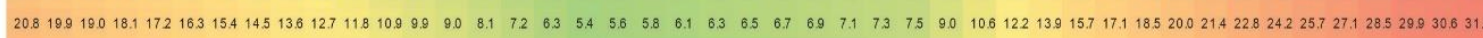

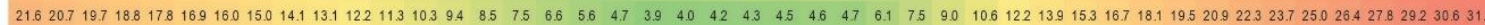

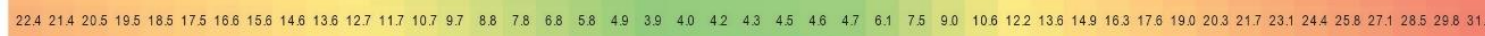

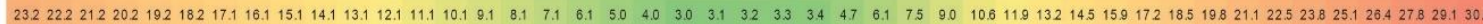

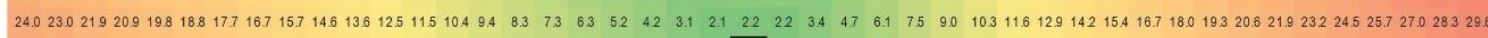

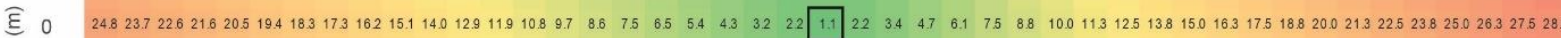

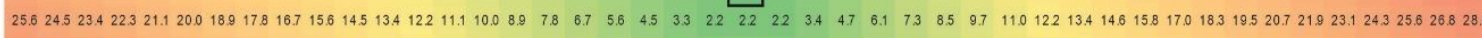

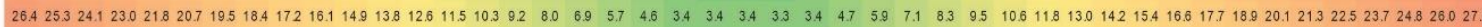

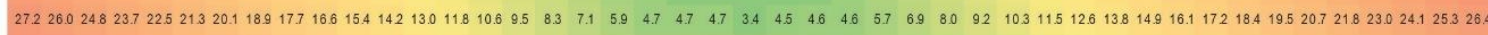

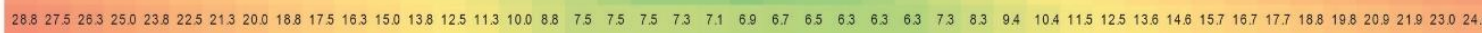

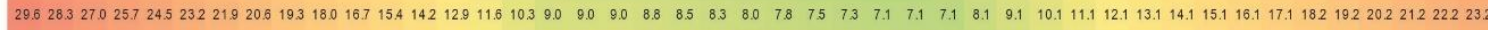

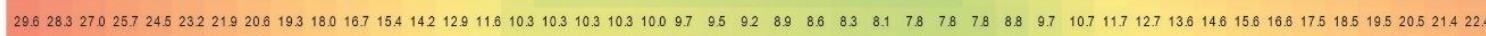

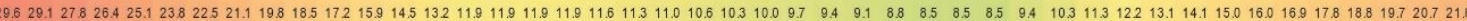

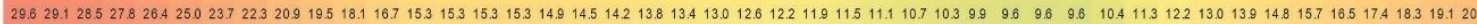

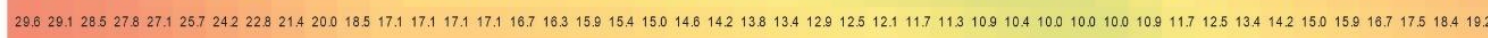
29.629 .128527 .827 .125 .724222 .821420 .018518 .518 .518 .518 .518 .117 .617216 .716 .315 .815414914 .514 .013 .613 .112 .712211811310 .910 .910910 .911712513 .414215 .015 .916 .717 .518419 .2

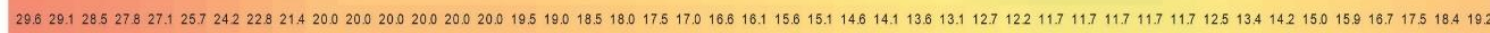

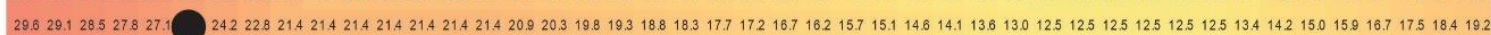

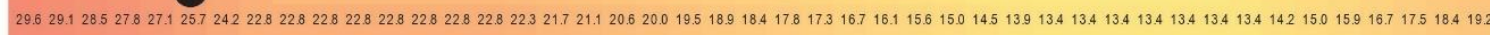
29.629 .128 .527 .827 .125724222 .822822822 .822 .822822 .822 .822 .321 .721 .120 .620 .019 .518918 .417 .817 .316 .716 .115 .615 .014 .513913 .413 .413 .413 .413 .413 .413 .414215 .015 .916 .717 .518419 .2 29.629 .128527 .827 .125724224224224224224224224224223 .723 .122 .521 .921320 .720 .119 .518 .918 .317717 .116 .616 .015 .414 .814214214214214214214214215 .0159196 .717 .518 .4192

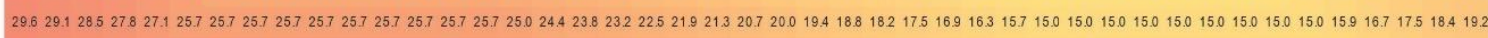

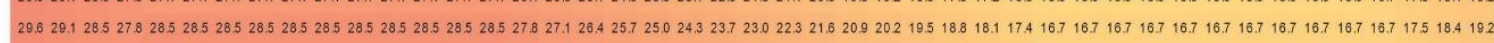

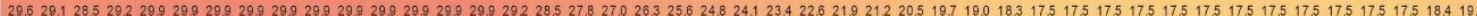

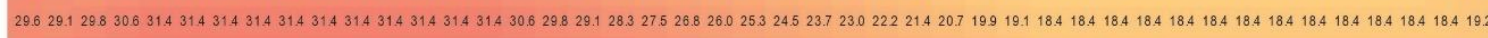

Figure 3.9 - Surface view of the soil grid of Scenario 4. The grid is identical to the base grid of Scenario 1, but is weighted by being multiplied by a separate grid of values (not shown). The two black dots represent positions of the highest weighting. The black dots are the spatial locations where the base grid is multiplied by two and these are intended to be the locations of the greatest directional bias (i.e. where the roots are), but since the original high values of the base grid are also weighted (though, by smaller numbers), they still maintain their capacity to modify growth direction. This can be seen in the fact that high values are still present at the periphery. 


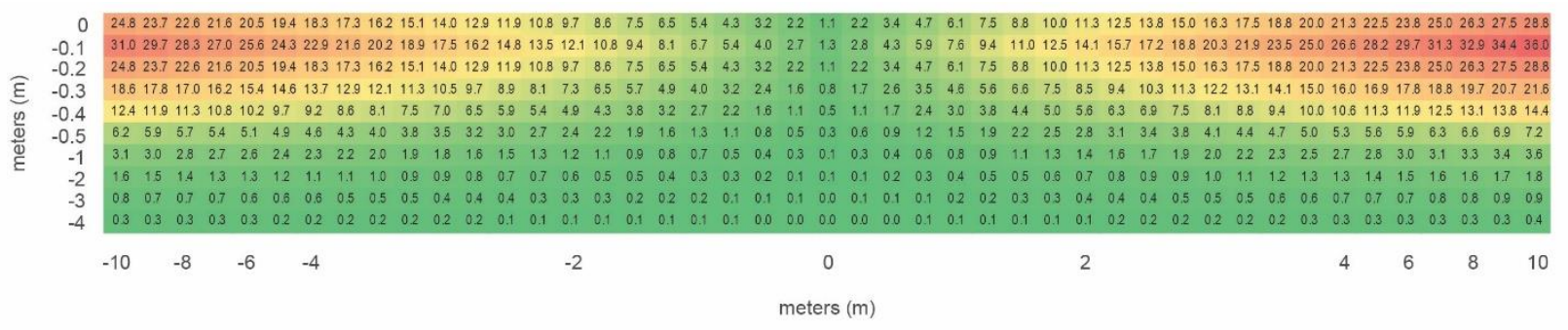

Figure 3.10 - Profile view of the soil grid of Scenario 4. The grid is identical to the base grid of Scenario 1, but is weighted by being multiplied by a separate grid of values. Since the original high values of the base grid are weighted (though, by smaller numbers), they still maintain their capacity to modify growth direction and the original overall distribution of high and low directional biases is maintained. Thus, this graphic is very similar to that of the profile view of Scenario 1. 


\section{$\underline{3.3 \text { - Results }}$}

Surface and profile visualizations of the output root system are used to demonstrate and discuss the architectural response to the soil grids created for each scenario. The root system is assessed visually, to qualitatively determine if roots react to tropisms in each scenario by avoiding or failing to grow in areas of impedance, or opportunistically growing towards directional biases. Profile views demonstrate examples of rooting depth and distribution. In addition, a $20 \mathrm{~m}$ x $20 \mathrm{~m}$ grid of cells is used to average root axis depth over the entirety of the root system. The average depth is an average of the $\mathrm{z}$ values (depth values) of root axis vertices that fall within the cell. Root axis vertices describe the spatial co-ordinate of every $1 \mathrm{~cm}$ length of the root axis. Depth results are used to display averaged depth response to obstacles or areas of attraction.

Averaged root diameter measurements at defined distance intervals are used to demonstrate the formation of a ZRT. The average root diameter is calculated at defined distance intervals of $0.25 \mathrm{~m} ; 0.5 \mathrm{~m} ; 0.75 \mathrm{~m} ; 1 \mathrm{~m} ; 1.5 \mathrm{~m} ; 2 \mathrm{~m}$, and at every subsequent $1 \mathrm{~m}$ increment until10m distance is reached. Distance intervals are expressed as concentric rings. Rates of decrease in root diameter, which underpin the ZRT measure, are a function of distance from the base of the tree.

For each scenario, Rootbox is run five times to account for random variations in how the root systems arrange themselves vertically and horizontally. Except for root diameter, no architectural parameters change between or during the simulations or scenarios. In all scenarios, there is a clear tendency of the root system to grow outwards and slightly downwards in the first 1 to $2 \mathrm{~m}$ horizontal distance from the tree base, and then rise again - often touching the surface - after this point. This indicates that the basic grid influences the shape of the root system in all cases. In Scenario 2, the root system stops growing once it reaches the low grid values under the sidewalk, while some roots dive under the area of compaction to reach the other side of the sidewalk. Despite growing down and under the sidewalk, the sidewalk cuts off most root growth and creates a noticeable imbalance of the number of roots between either side of the sidewalk. In Scenario 3, the nutrient or water conduit draws roots towards it and under it, though this would seem to apply only to roots that grow within $0.75 \mathrm{~m}$ distance from the nutrient source, which is the distance at which grid values were scaled higher. Scenario 4 does not result in any noticeable bias towards the input weights and produced both architectural and depth attributes that are almost identical to those produced in Scenario 1. 
In all scenarios, a ZRT occurs in the first $1 \mathrm{~m}$ of horizontal distance from the base of the tree, which is a small underestimation of values reported in the literature. In this case, the ZRT is calculated as the distance at which the rate of change in average root diameter exceeds $1.25 \%$ per centimeter distance. Generally, there are differences in the distribution of the rate of taper between each scenario and in the average root diameters measured at each distance interval. 


\subsubsection{Scenario 1 - Base Grid}

The base grid scenario introduces uniform soil tropism towards the upper $0.5 \mathrm{~m}$ of the soil and towards the outer edges of the grid. Depths below this point become progressively smaller and after $1 \mathrm{~m}$ depth begins to hinder growth by reducing root extension. In one of the model iterations, the horizontal distribution of the roots is highly skewed to one direction. This is due to the random horizontal angle of the starting point of the root. However, in four out of the five iterations, roots tend to evenly distribute around the tree base, and so tend to grow outwards in all directions. Figure 3.11 shows the distribution pattern of the $5^{\text {th }}$ iteration, as an example. The maximum horizontal distance for all iterations is approximately $9.5 \mathrm{~m}$. If the tree in this case is assumed to be $0.25 \mathrm{~m} \mathrm{DBH}$ then the ratio of DBH to maximum spread is $1: 38$, which is consistent with the literature (Day et al., 2010).

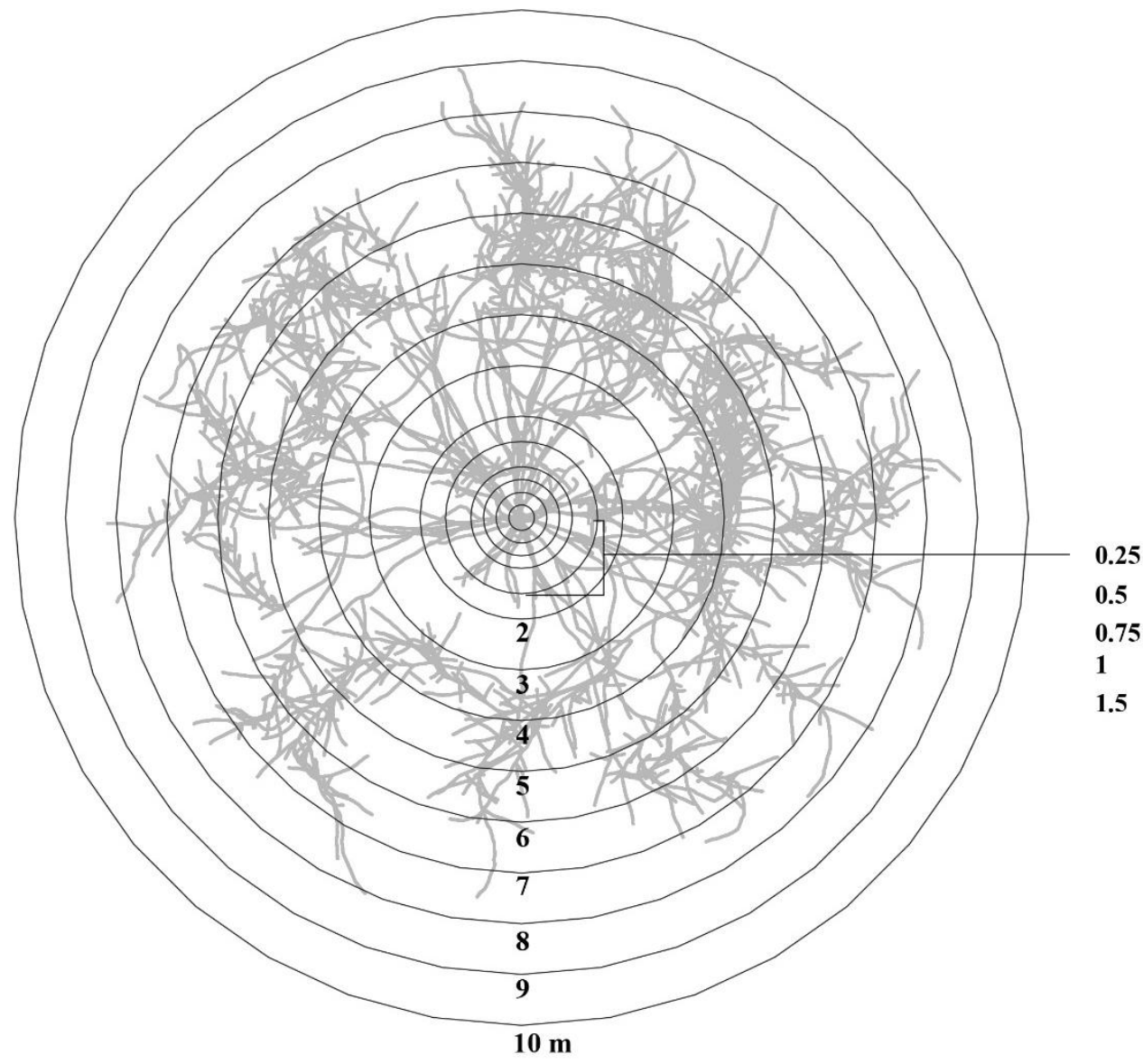

Figure 3.11 - Surface distribution of the $5^{\text {th }}$ simulation iteration (Scenario 1). Concentric rings are the distance intervals used to sample average diameters for ZRT identification. 
In Figure 3.12 and 3.13, most roots remain at or grow towards the top 0 to $0.1 \mathrm{~m}$ of soil. Roots which start growing vertically will dive deeper, and may reach beyond $1 \mathrm{~m}$ in depth, but then gradually move towards the surface. In any case, the number of roots that dive deeper than $0.5 \mathrm{~m}$ is relatively small, and most roots of smaller diameter tend to occur between 0 to $0.5 \mathrm{~m}$ in depth.

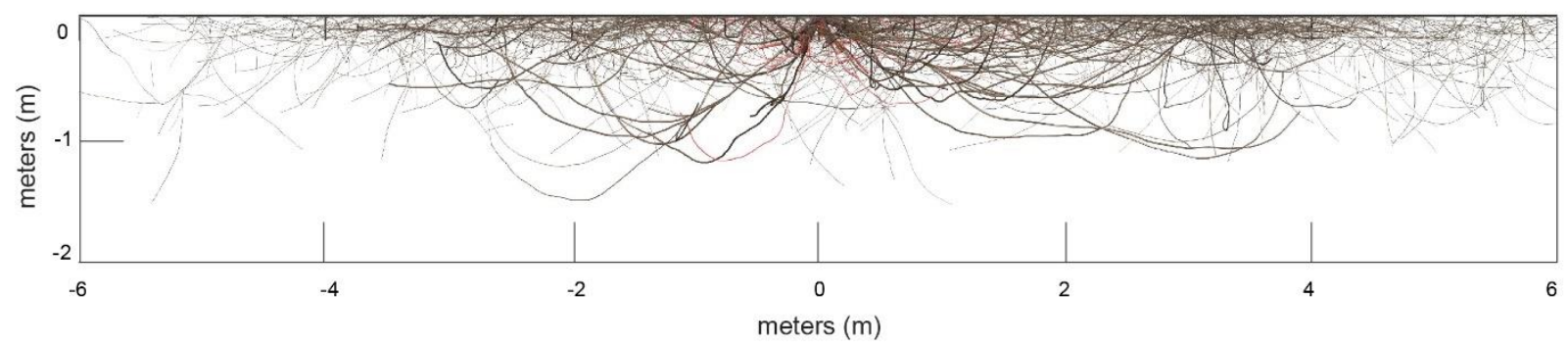

Figure 3.12 - Profile of the $5^{\text {th }}$ simulation iteration (Scenario 1). The graphic is produced in MATLAB and is an elevation profile of the root system, which means that all the roots of the system are being displayed from a side view. 


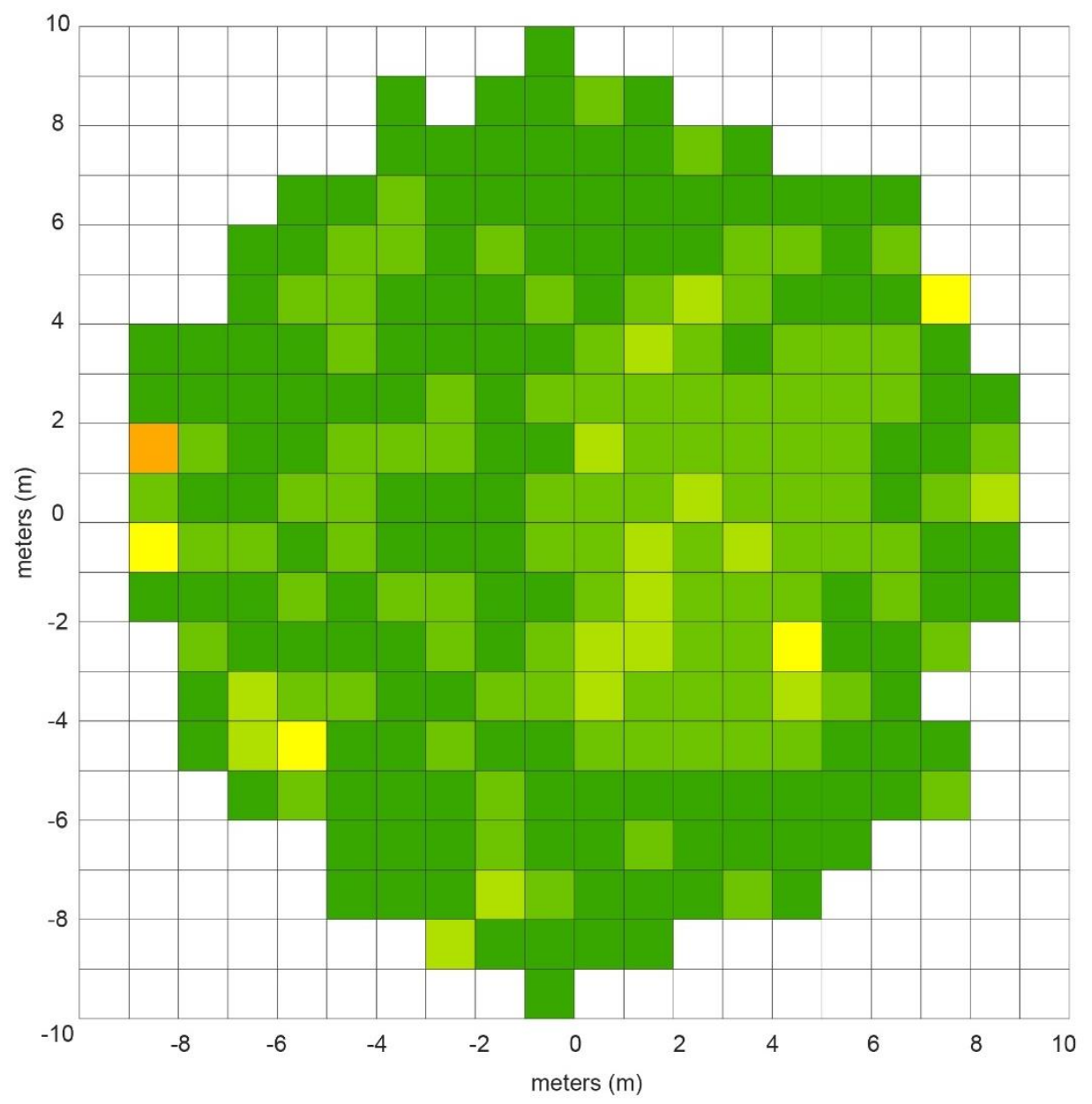

Average depth (m)

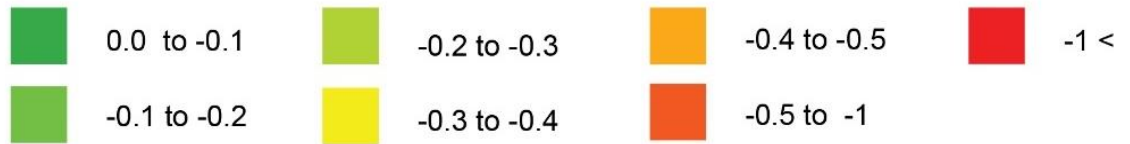

Figure 3.13 - Scenario 1 average depth of root vertices (i.e. the three-dimensional points along a root axis that describe its location and topological and geometric attributes). Depth values are averaged over a $1 \mathrm{~m}$ by $1 \mathrm{~m}$ grid cell and between all five simulation iterations, so maximum rooting depths, which can exceed $1.5 \mathrm{~m}$ depth in Scenario 1, are smoothed in this analysis.

The ZRT ends at the $0.75 \mathrm{~m}$ distance interval (see Table 3.2). At all distance intervals between 0 and $0.5 \mathrm{~m}$, the rate of change in average diameter per centimeter exceeds $1.25 \%$, but the most rapid taper is between 0 and $0.25 \mathrm{~m}$ distance, measured at approximately $2.84 \%$ change per centimeter increment. 
Table 3.2 - Scenario 1 root diameter taper statistics. In the first column, distance from the tree base is indicated. In the second column, average root diameter is shown, which indicates the averaged values of all roots that cross the concentric ring at that distance. The third column, indicates the rate of change in the average root diameter between the current and the previous distance interval. The fourth column indicates the percent change in average root diameter per centimeter distance, which allows for determination of where the ZRT ends, and this point is indicated with a '*' symbol.

\begin{tabular}{|l|l|l|l|}
\hline Distance & $\begin{array}{l}\text { Average } \\
\text { Diameter }(\mathbf{c m})\end{array}$ & $\begin{array}{l}\text { Percent }(\%) \text { change in } \\
\text { average diameter }\end{array}$ & $\begin{array}{l}\text { Percent }(\%) \text { change in average diameter, per } \\
\text { cm distance }\end{array}$ \\
\hline $0 \mathrm{~m}$ & 6 & & \\
\hline 0.25 & 1.73077 & 71.15 & 2.846 \\
\hline 0.5 & 1.02257 & 82.96 & 1.659 \\
\hline 0.75 & 0.783188 & 86.95 & $1.159 *$ \\
\hline 1 & 0.726654 & 87.89 & 0.879 \\
\hline 1.5 & 0.661635 & 88.97 & 0.593 \\
\hline 2 & 0.540008 & 91.00 & 0.455 \\
\hline 3 & 0.529242 & 91.18 & 0.304 \\
\hline 4 & 0.340233 & 94.33 & 0.236 \\
\hline 5 & 0.262992 & 95.62 & 0.191 \\
\hline 6 & 0.25776 & 95.70 & 0.160 \\
\hline 7 & 0.261356 & 95.64 & 0.137 \\
\hline 8 & 0.262424 & 95.63 & 0.120 \\
\hline 9 & 0.2794 & 95.34 & 0.106 \\
\hline
\end{tabular}

\subsubsection{Scenario 2 - Nutrient Source or Leaky Pipe}

The nutrient source or leaky pipe scenario uses the base grid of Scenario 1, but introduces an area of higher directional bias up to $0.75 \mathrm{~m}$ on either side, and down to a $1 \mathrm{~m}$ depth, of the location of the source. The values within this envelope are the basic (Scenario 1) grid values multiplied by three. Figure 3.14 shows the distribution pattern of the $5^{\text {th }}$ iteration, but there is no discernable horizontal growth bias towards the source in any of the iterations. Like Scenario 1, the maximum root spread is approx. $9.5 \mathrm{~m}$. 


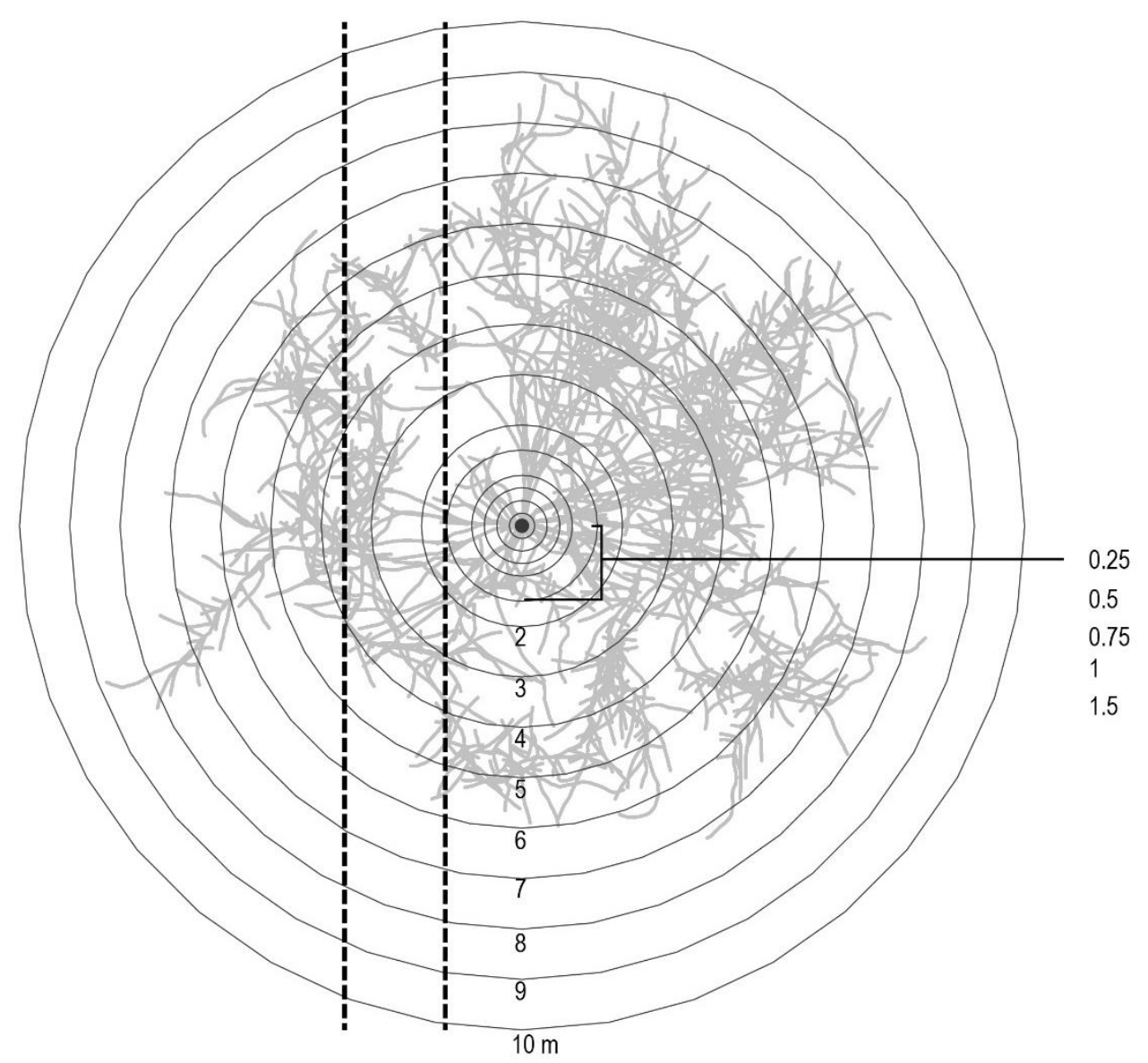

Figure 3.14 - Horizontal distribution of the $5^{\text {th }}$ simulation iteration (Scenario 2). Concentric rings are the distance intervals used to sample average diameters for ZRT identification. The two outer boundaries of the nutrient/water conduit are indicated with dashed lines.

Moreover, the vertical profile of the roots does show a clear directional bias downwards near the source. Roots dive deeper into the soil, to a maximum of $3 \mathrm{~m}$ depth. In all scenarios, root vertices below $0.5 \mathrm{~m}$ depth occur more frequently near the source than anywhere else (see Figures 3.15 and 3.16). 


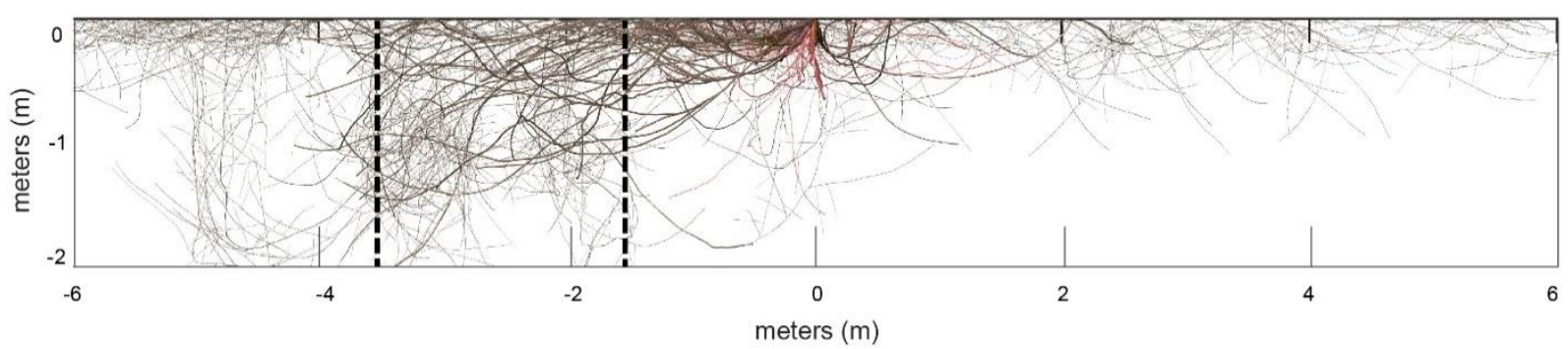

Figure 3.15 - Profile of root distribution of the $5^{\text {th }}$ simulation iteration (Scenario 2). The graphic is produced in MATLAB and is an elevation profile of the root system, which means that all the roots of the system are being displayed from a side view. The two outer boundaries of the nutrient/water conduit are indicated with dashed lines. 


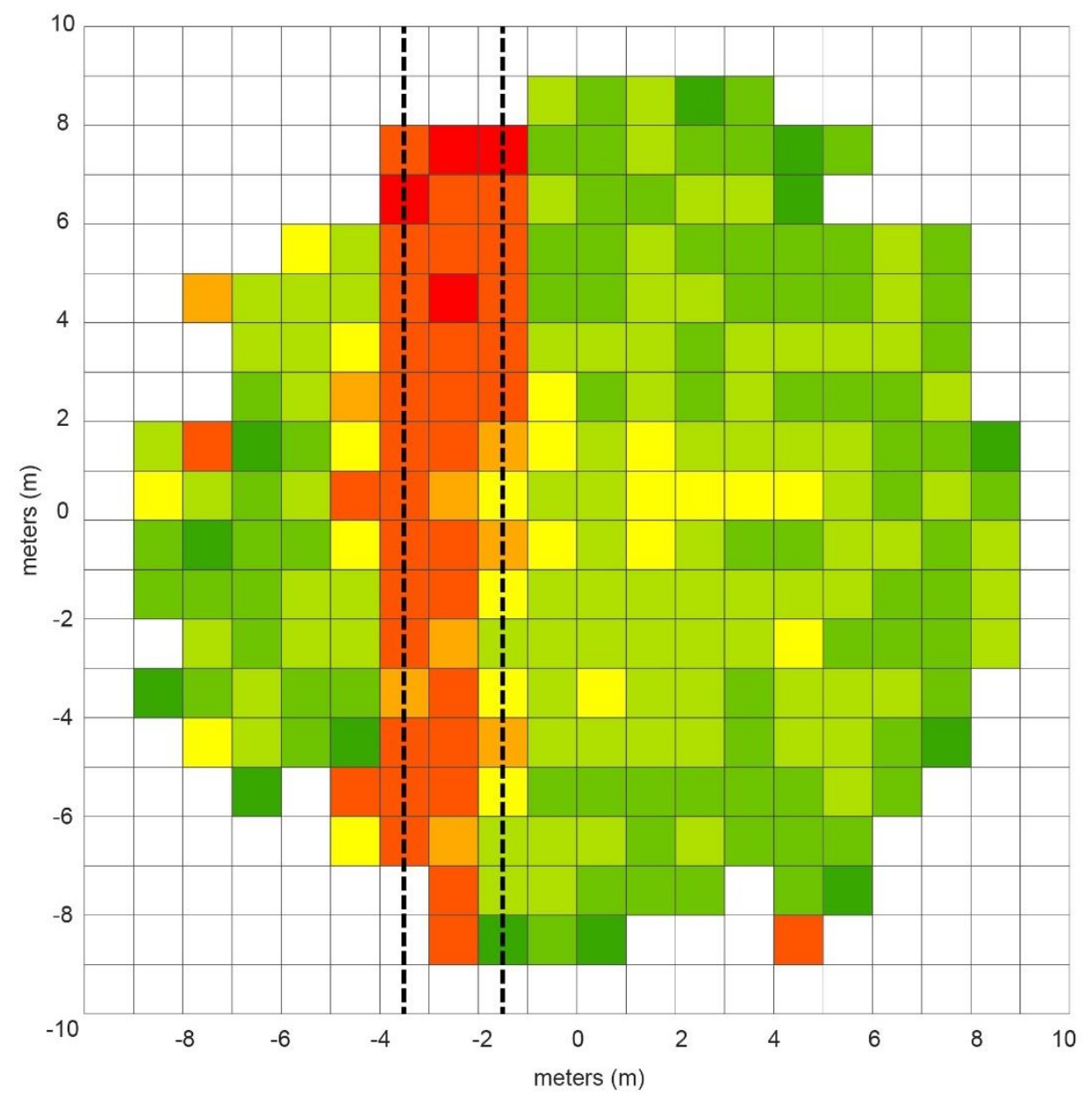

Average depth (m)

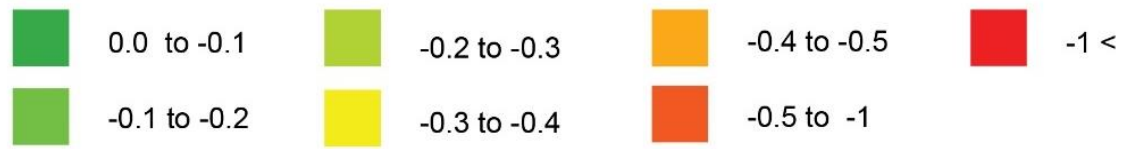

Figure 3.16 - Scenario 2 average depth of root vertices (i.e. the three-dimensional points along a root axis that describe its location and topological and geometric attributes). Depth values are averaged over a $1 \mathrm{~m}$ by $1 \mathrm{~m}$ grid cell and between all five simulation iterations, so maximum rooting depths are smoothed in this analysis. The two outer boundaries of the nutrient/water conduit are indicated with dashed lines.

Similar to Scenario 1, the ZRT ends at the $0.75 \mathrm{~m}$ distance interval (see Table 3.3). At all distance intervals between 0 and $0.75 \mathrm{~m}$, the rate of change in average diameter per centimeter exceeds $1.25 \%$, but the most rapid taper is between 0 and $0.25 \mathrm{~m}$ distance, measured at approx. $2.55 \%$ change per centimeter increment. 
Table 3.3 - Scenario 2 root diameter taper statistics. In the first column, distance from the tree base is indicated. In the second column, average root diameter is shown, which indicates the averaged values of all roots that cross the concentric ring at that distance. The third column, indicates the rate of change in the average root diameter between the current and the previous distance interval. The fourth column indicates the percent change in average root diameter per centimeter distance, which allows for determination of where the ZRT ends, and this point is indicated with a '*' sign.

\begin{tabular}{|l|l|l|l|}
\hline Distance & $\begin{array}{l}\text { Average } \\
\text { Diameter } \\
(\mathbf{c m})\end{array}$ & $\begin{array}{l}\text { Percent }(\%) \text { change in } \\
\text { average diameter }\end{array}$ & $\begin{array}{l}\text { Percent }(\%) \text { change in } \\
\text { average diameter, per cm } \\
\text { distance }\end{array}$ \\
\hline $0 \mathrm{~m}$ & 6 & & \\
\hline $0.25 \mathrm{~m}$ & 2.171 & 63.822 & 2.55 \\
\hline 0.5 & 1.234 & 79.438 & 1.59 \\
\hline 0.75 & 0.999 & 83.345 & $1.11^{*}$ \\
\hline 1 & 1.026 & 82.892 & 0.83 \\
\hline 1.5 & 1.080 & 82.004 & 0.55 \\
\hline 2 & 0.686 & 88.571 & 0.44 \\
\hline 3 & 0.546 & 90.904 & 0.30 \\
\hline 4 & 0.362 & 93.961 & 0.23 \\
\hline 5 & 0.270 & 95.504 & 0.19 \\
\hline 6 & 0.262 & 95.632 & 0.16 \\
\hline 7 & 0.268 & 95.537 & 0.14 \\
\hline 8 & 0.280 & 95.326 & 0.12 \\
\hline 9 & 0.320 & 94.663 & 0.11 \\
\hline
\end{tabular}

\subsection{3 - Scenario 3 - Sidewalk (Physical Barrier) Underlaid with Compacted Soil}

The sidewalk obstacle and compaction scenario also uses the base grid of Scenario 1, but introduces an area of very low directional bias approximately $1 \mathrm{~m}$ away from the tree base and down to $0.4 \mathrm{~m}$ depth. In all iterations, there is a discernable horizontal reduction in the number of roots on the far side of the obstacle when compared with the near side (where the base of the tree is located), to a depth of approximately $0.5 \mathrm{~m}$ (Figure 3.17 ). This is borne out by how roots are distributed vertically. In the profile view (Figure 3.18) many roots fail to grow past the barrier, while those that grow underneath grow upwards after emergence on the other side. Because the barrier is located near the soil surface, it impedes the large number of roots that tend to grow in the top layers of the soil. In addition, the average depth of root vertices in this location is much deeper, and roots in this area are much deeper than surrounding roots. 


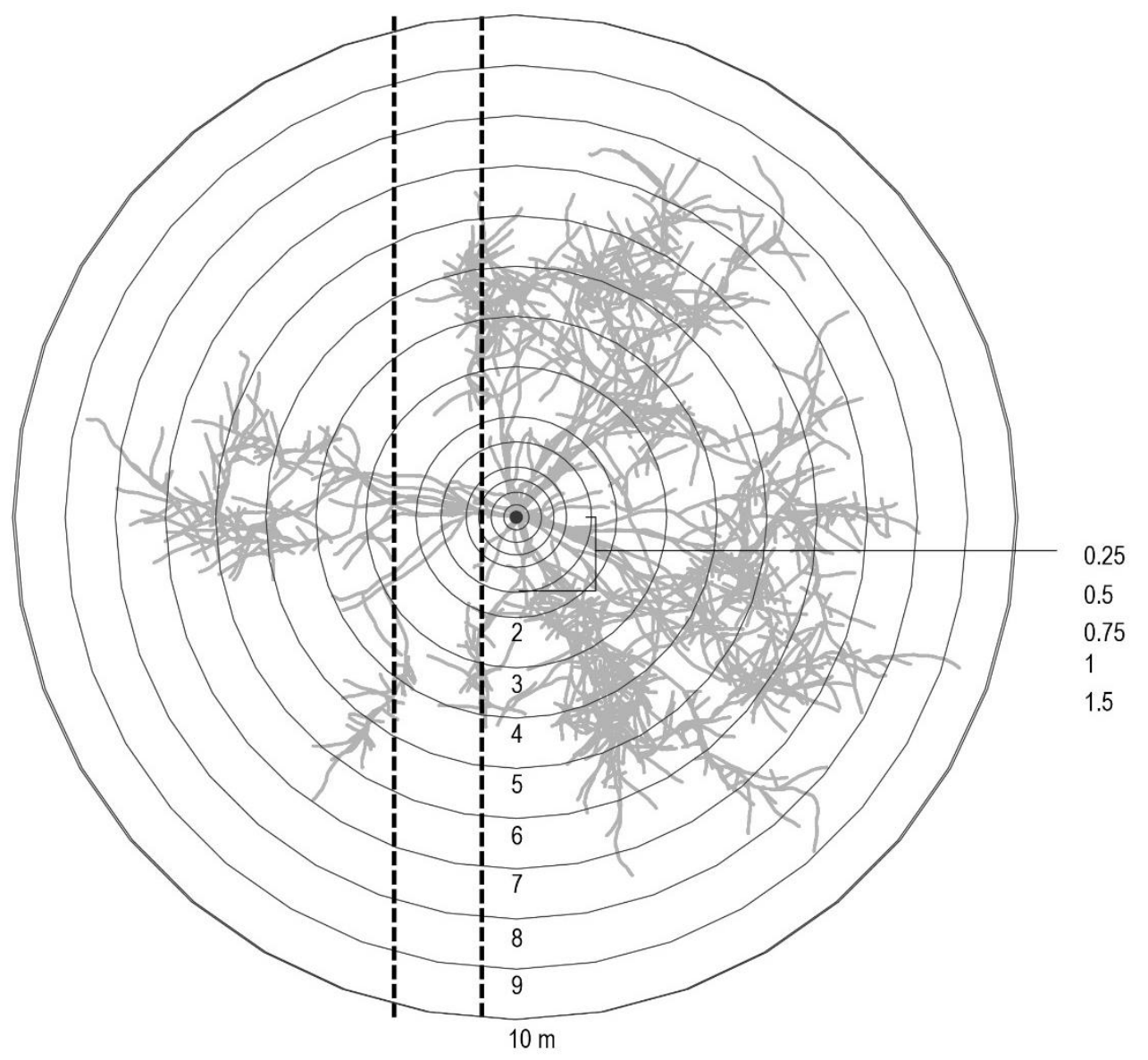

Figure 3.17 - Horizontal distribution of the $5^{\text {th }}$ simulation iteration (Scenario 3). Concentric rings are the distance intervals used to sample average diameters for ZRT identification. The two outer boundaries of the sidewalk are indicated with dashed lines.

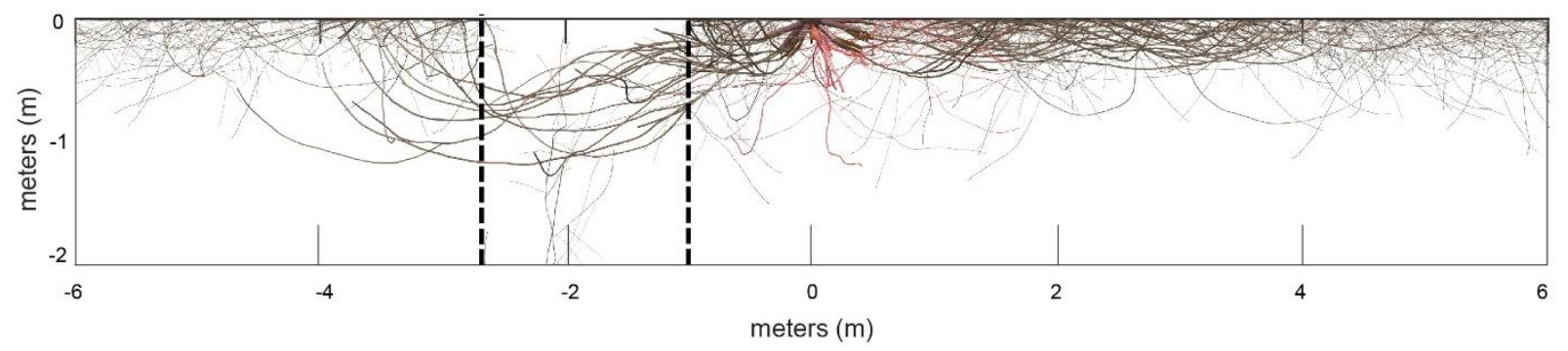

Figure 3.18 - Vertical distribution (below ground) of the $5^{\text {th }}$ simulation iteration (Scenario 3). The graphic is produced in MATLAB and is an elevation profile of the root system, which means that all the roots of the system are being displayed from a side view. The two outer boundaries of the sidewalk are indicated with dashed lines. 


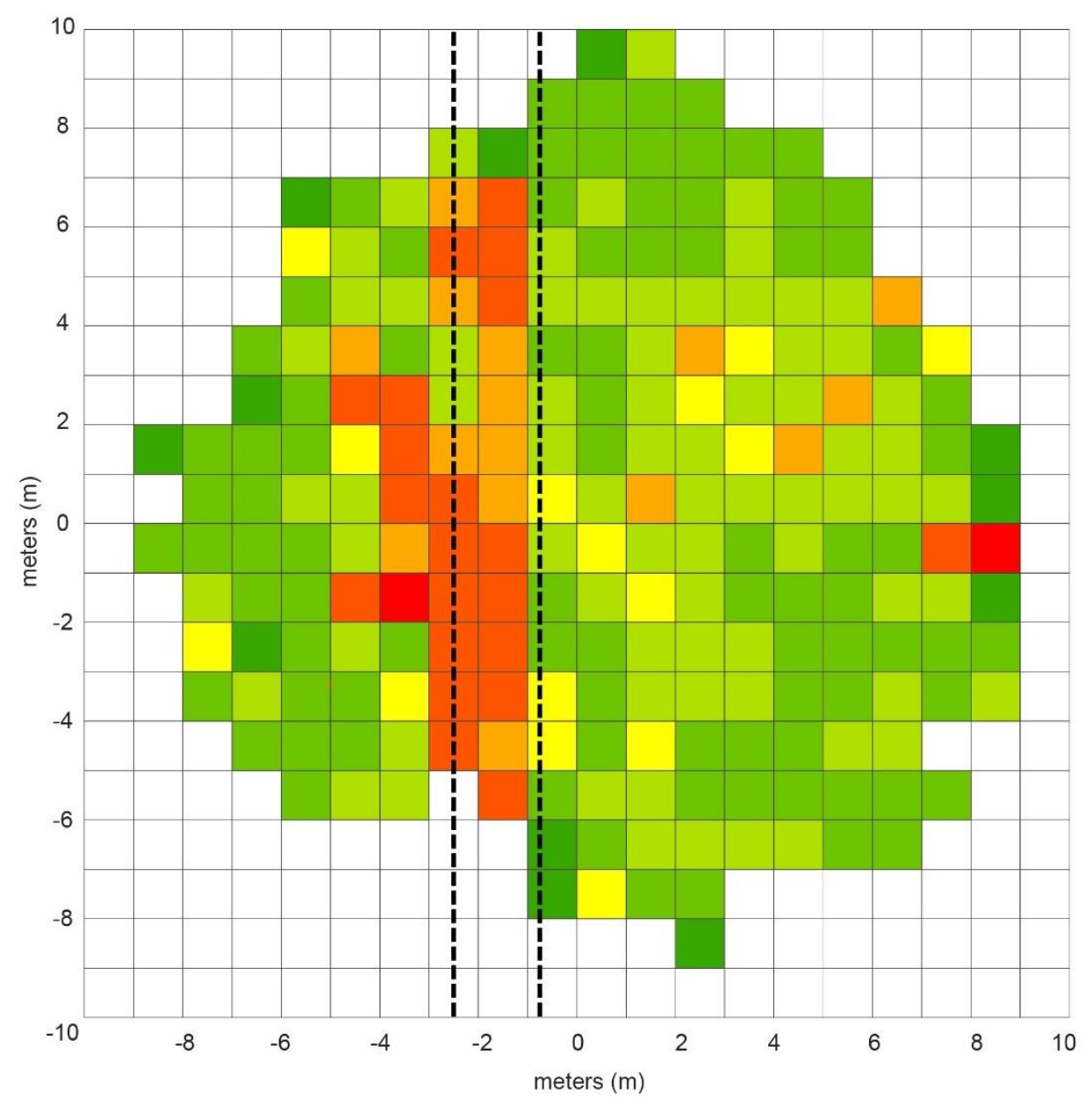

Average depth (m)

0.0 to -0.1

-0.2 to -0.3

-0.4 to -0.5

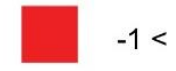

-0.1 to -0.2

-0.3 to -0.4

-0.5 to -1

Figure 3.19 - Scenario 3 average depth of root vertices (i.e. the three-dimensional points along a root axis that describe its location and topological and geometric attributes). Depth values are averaged over a $1 \mathrm{~m}$ by $1 \mathrm{~m}$ grid cell and between all five simulation iterations, so maximum rooting depths are smoothed in this analysis.

Like Scenarios 1 and 2, the ZRT ends at the $0.75 \mathrm{~m}$ distance interval (see Table 3.4). The most rapid taper is between 0 and $0.25 \mathrm{~m}$ distance, and is approximately $2.35 \%$ change per centimeter increment. 
Table 3.4 - Scenario 3 root diameter taper statistics. In the first column, distance from the tree base is indicated. In the second column, average root diameter is shown, which indicates the averaged values of all roots that cross the concentric ring at that distance. The third column, indicates the rate of change in the average root diameter between the current and the previous distance interval. The fourth column indicates the percent change in average root diameter per centimeter distance, which allows for determination of where the ZRT ends, and this point is indicated with a '*' sign.

\begin{tabular}{|c|c|l|l|}
\hline Distance & $\begin{array}{c}\text { Average } \\
\text { Diameter }\end{array}$ & $\begin{array}{c}\text { Percent (\%) change in average } \\
\text { diameter }\end{array}$ & $\begin{array}{c}\text { Percent (\%) change in average } \\
\text { diameter, per cm distance }\end{array}$ \\
\hline $0 \mathrm{~m}$ & 6 & & \\
\hline 0.25 & 2.477 & 58.719 & 2.349 \\
\hline 0.5 & 1.443 & 75.955 & 1.519 \\
\hline 0.75 & 1.305 & 78.255 & 1.043 \\
\hline 1 & 1.236 & 79.399 & 0.794 \\
\hline 1.5 & 1.201 & 79.988 & 0.533 \\
\hline 2 & 1.073 & 82.110 & 0.411 \\
\hline 3 & 0.772 & 87.131 & 0.290 \\
\hline 4 & 0.462 & 92.299 & 0.231 \\
\hline 5 & 0.271 & 95.475 & 0.191 \\
\hline 6 & 0.264 & 95.596 & 0.159 \\
\hline 7 & 0.269 & 95.519 & 0.136 \\
\hline 8 & 0.285 & 95.256 & 0.119 \\
\hline 9 & 0.332 & 94.463 & 0.105 \\
\hline
\end{tabular}

\subsection{4 - Scenario 4 - Weighting the Basic Grid Based on In-Situ Knowledge of Root Location}

Since Scenario 4 weights the base grid of Scenario 1, ZRT and maximum spread results are not discussed for Scenario 4. It is assumed that these values are similar to those observed in Scenario 1.

From a visual assessment of the output architecture, there is no discernable overall difference between the averaged spatial distribution when Scenario 4 and Scenario 1 are compared (Figure 3.20 and 3.21). 


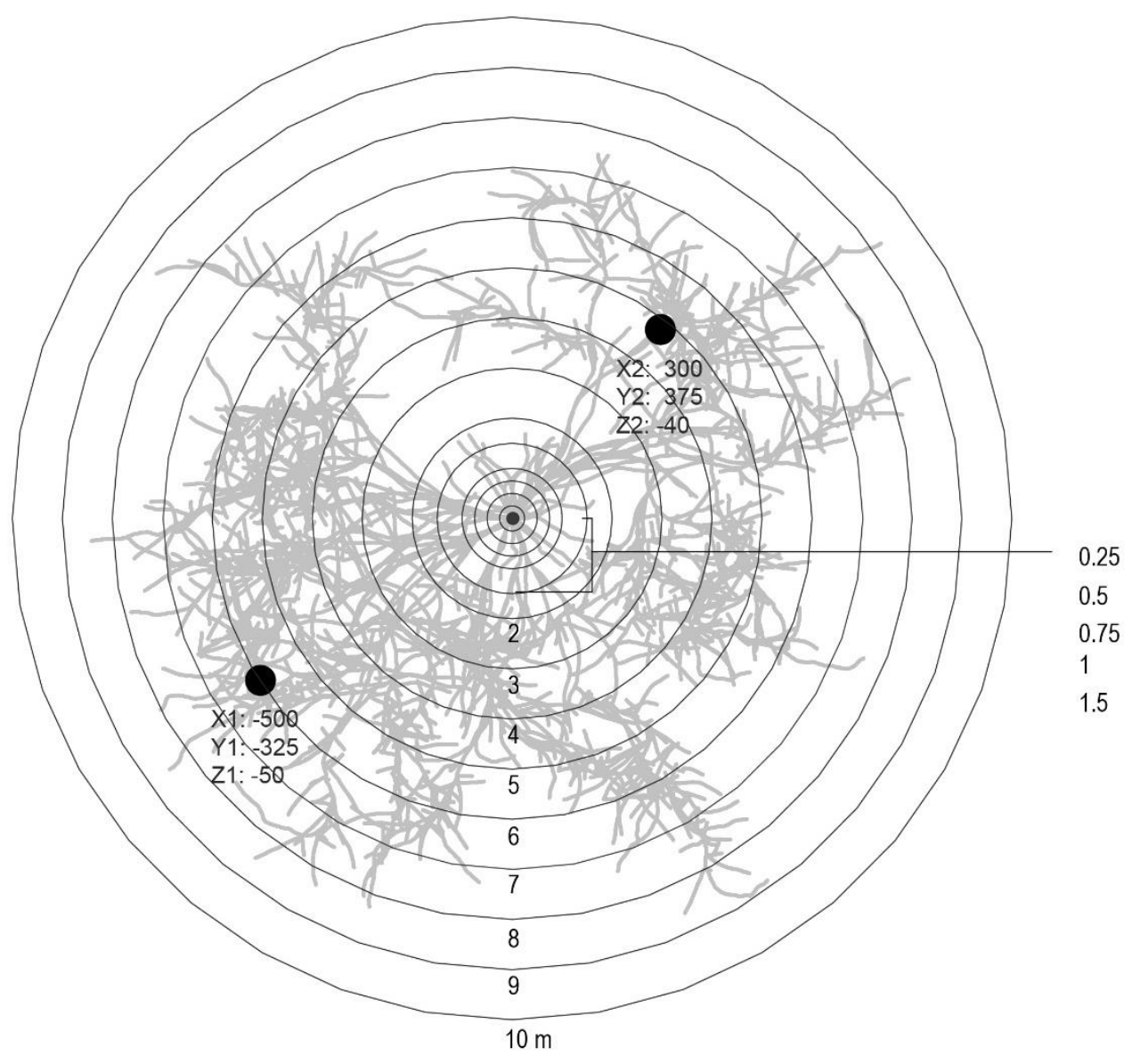

Figure 3.20 - Horizontal distribution of the $5^{\text {th }}$ simulation iteration (Scenario 4). Concentric rings are the distance intervals used to sample average diameters for ZRT identification, however in this scenario the ZRT nor root system spread is calculated. The two black dots indicate the (hypothetical) known locations of roots.

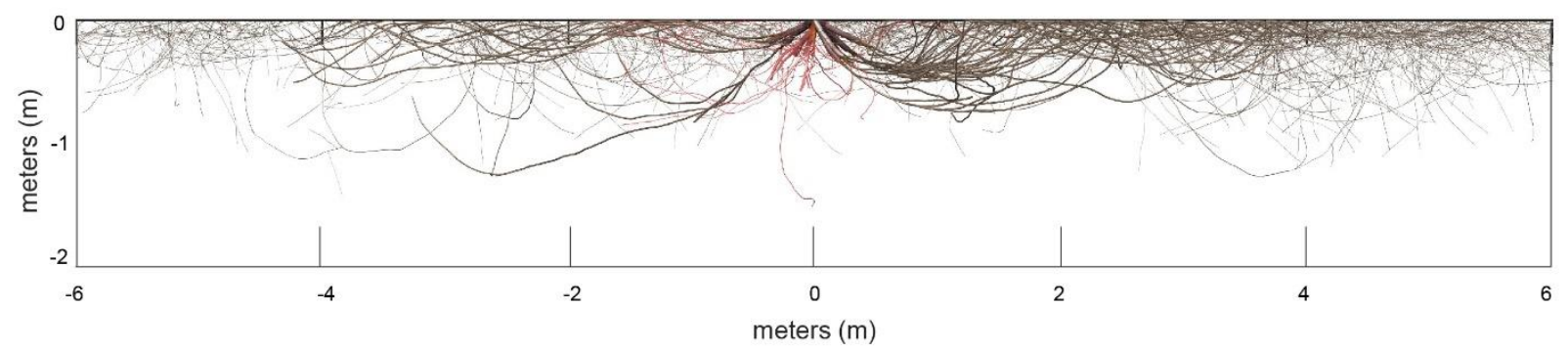

Figure 3.21 - Vertical distribution (below ground) of the $5^{\text {th }}$ simulation iteration (Scenario 4). The graphic is produced in MATLAB and is an elevation profile of the root system, which means that the all the roots of the system are being displayed from a side view.

However, Scenario 1, on average, tends to create slightly shallower roots than does Scenario 4. (Figure 3.22). In total, $54.7 \%$ (138 out of 252) of the gridded cells are above $0.2 \mathrm{~m}$ depth in 
Scenario 1, whereas in Scenario 4 it is 51.8\% (126 out of 243). About the same maximum depths are achieved in both scenarios: $0.39 \%$ ( 1 out of 252 ) cells and $0.82 \%$ ( 2 of 243 ) cells are below $0.5 \mathrm{~m}$ depth, respectively. There is no discernable movement towards the points of highest directional bias.

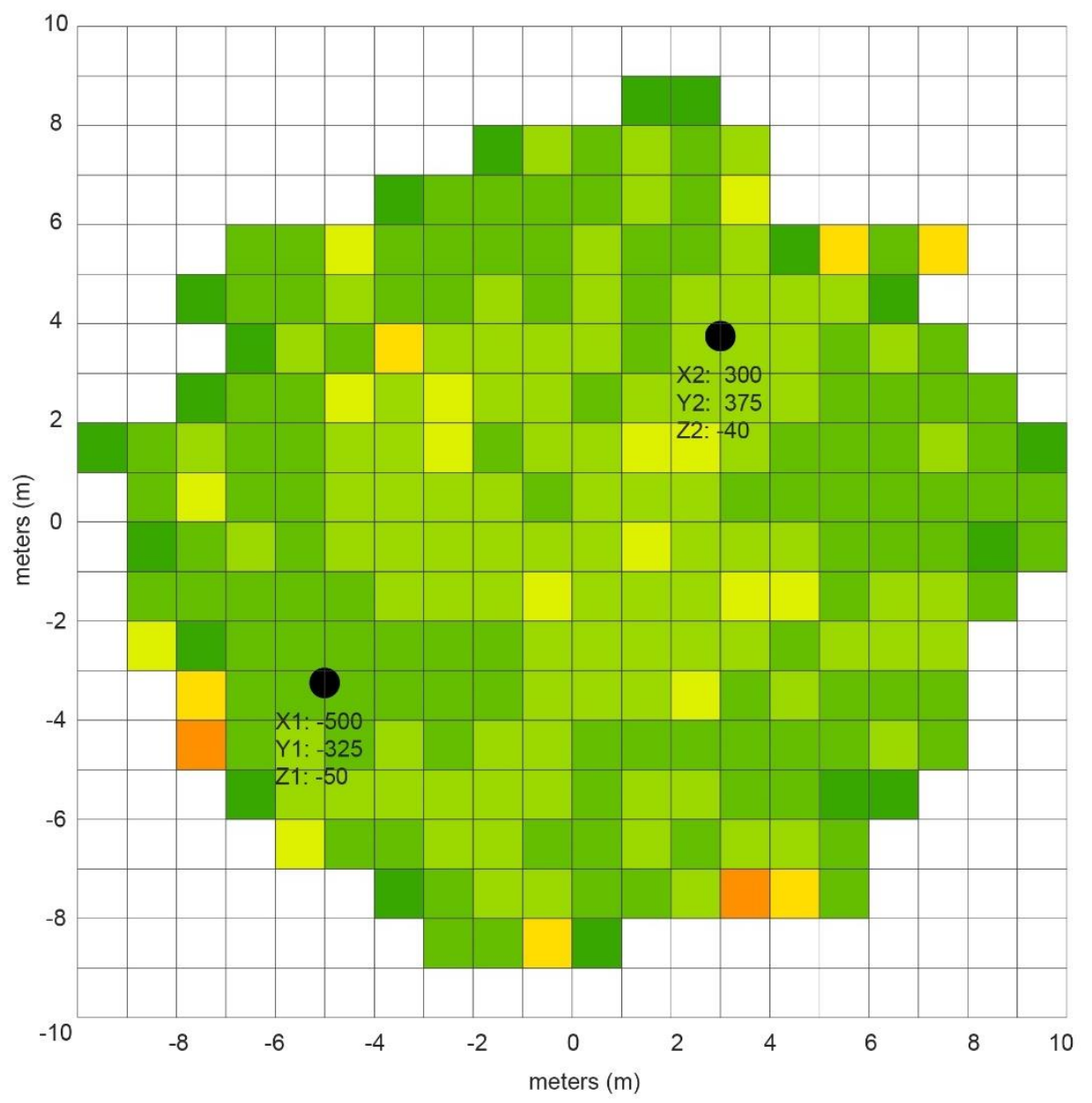

Average depth (m)
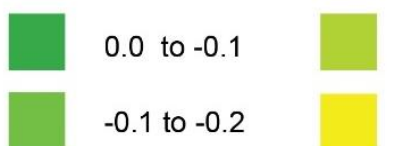

-0.2 to -0.3

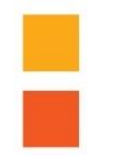

-0.4 to -0.5

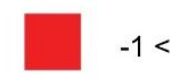

-0.3 to -0.4

-0.5 to -1

Figure 3.22 - Scenario 4 average depth of root vertices (i.e. the three-dimensional points along a root axis that describe its location and topological and geometric attributes). Depth values are averaged over a $1 \mathrm{~m}$ by $1 \mathrm{~m}$ grid cell and between all five simulation iterations, so maximum rooting depths are smoothed in this analysis. The black dots intended to be the locations (indicated in adjacent spatial co-ordinate notations) of known roots and therefore the greatest directional bias. Both $\mathrm{Z} 1$ and $\mathrm{Z} 2$ are located at $0.5 \mathrm{~m}$ depth and $0.4 \mathrm{~m}$ depth, respectively, but the average depths of root vertices in these locations do not exceed $0.3 \mathrm{~m}$. 


\section{4 - Discussion}

The present study demonstrates an approach to modelling the spatial interaction between a tree root system's architecture and several hypothetical urban soil environments. Moreover, output is compared with literature describing tree root architecture as a means of determining model performance to simulate realistic tree root architecture. To do this, the model should be able to: (1) simulate a realistic ZRT that is supported by observations in the literature; (2) mimic a vertical and horizontal spatial distribution of roots where most roots stay within $0.5 \mathrm{~m}$ of the surface; and (3) enable the creation of simplified soil environments that represent persistent site conditions that bias the three-dimensional configuration of root system growth and architecture.

The model output's ZRT is established by averaging diameter values over the five modelling iterations. Root spread is established by taking the maximum of any of the five modelling iterations. Root system shape is assessed both visually and

\subsection{1 - ZRT and Root Diameter}

For each of the first three scenarios, the average diameter of all roots within the first $0.75 \mathrm{~m}$ exhibited a high rate of taper. The rate conforms to the rate that has been proposed by Danjon, et al. (2005). Here, the ZRT is defined as that distance along the axis of the root that the rate of diameter change per centimeter is greater than $1.25 \%$. However, the authors had originally applied the definition to individual root axes, no matter the direction that the root is oriented, whereas the present study measures the change in the average diameter at defined intervals over a horizontal distance. Whichever way the ZRT is measured, the simulations somewhat underestimate the size reported in the literature values, however this is only due to parameter settings used in these simulations which could be further modified to increase the size of the ZRT.

While a distinct ZRT is successfully simulated, setting the parameters to produce one is done indirectly through the creation of a succession of several short roots near the base of the tree, spanning a maximum of 1 to $2 \mathrm{~m}$ in length, where each successor root has an incrementally smaller diameter than its predecessor. Rootbox does not directly allow for root thickening, though the program can be modified to include such a rule (Leitner, et al. 2010a). During a 
simulation, young roots begin growing with the same diameter they will have at the end of the simulation. This is a limitation of the model that could be improved in future work. Root thickening is a distinct characteristic found in, especially, older trees, (Coutts, 1987; Wagner et al., 2011), and occurs as the root ages over the course of several years or as a response to mechanical impediments. Parameters that allow thickening, and allow it to happen in response to changes in the soil environment over time, would yield a more realistic architecture and would be an important step in creating functional-structural model of tree root systems.

Rootbox does not allow for a root collar (i.e. the base of the tree) to be created. Root collar diameter is important because it influences the establishment of the final radius of the ZRT and total root spread. In Rootbox, basal / first-order roots grow from the same origin point $(0,0,0)$. The root collar diameter may be incorporated indirectly by making a short-length, verticallyoriented root with a large diameter, but this approach can be cumbersome because the root requires a sufficiently large apical zone from which successors could branch, and this is difficult to achieve on a short-length root.

\subsection{2 - Root System Shape}

To facilitate comparison between scenarios, root growth parameters were kept constant between the simulations. Besides stochastic variability in root diameter, length and successor number parameters are not allowed to vary, which narrows the range of variability of depth and horizontal spread of the root system between simulations. In each output the maximum rooting depth of individual roots varied as some roots, likely on trajectories determined by their preceding parent-roots, dive down to penetrate below $1 \mathrm{~m}$ in depth. This pattern is not the result of any "sinker"-type roots specified in advance (Armstrong et al., 1976; Coutts 1987; Danjon et al., 2008), but are rather a product of the model's inherent stochastic behaviour during simulation. In the real-world, urban settings, some roots are known to grow to lower depths to access the hydrological resources which are in short supply at the surface (Day and Wiseman, 2010). But, in the case of the model outputs, downwards growth patterns are determined mainly by the initial angles at which the roots begin to grow from their predecessors and their ability to stray from that angle during extension. The movement downwards is not an intentional output of the model, such as a gravitropism function (Ge et al., 2000; Rigas et al., 2013; Walk et al., 2006). 
Instead, the movement of some roots to lower depths is the result of random and inexplicable variations in root growth direction during simulation, and this kind of behaviour is inherent in the Rootbox model. While stochastic output is a potential weakness of the model, it could be argued that this kind of inexplicable variation is advantageous because the ontogeny of some root architecture patterns may only be due to random, uncontrolled variation (Forde, 2009) in growth patterns. Random variation in model output can also account for biological and environmental processes that are poorly understood (Dunbabin et al., 2013). However, this contributes to a lack of user control over the model's output and is a major limitation to efforts to couple field measurements (e.g. known root locations or the cardinal directions of root initiation at the tree base) to simulated growth.

The maximum horizontal distance of the spread of the root system is approximately $9 \mathrm{~m}$. This is a maximum that is achieved, for any one root, in any of the five model iterations that were conducted. Remarkably, for each of the scenarios, maximum horizontal root spread is consistently reached at $9 \mathrm{~m}$. The consistency is likely due to length controlling parameters that will terminate root growth at the same distance in every simulation. However, the maximum distance of any individual root potentially does not indicate the maximum root spread of the entire root system, which is an attribute that is probably of more significance to studies that wish to predict the maximum extent of the roots. This is a limitation of this research that could potentially be overcome in future work by averaging the maximum lengths of the root axis, measured as the distance traversed from the initial emergence of the root at the tree's base to the terminal point (i.e. the tip) (Danjon et al., 2008). This approach to root axis length was not pursued in this research because of difficulties in establishing unique identifiers for each root axis in the model's ouput.

Model runs of the same scenario produced noticeable variations in root spatial distribution in the horizontal plane. In any of five model runs for one scenario, root growth may evenly distribute around the tree base or could favour a dominant direction. This variation in growth direction is due to differences in the initial horizontal angle (measured from the parent's axis) of the firstorder roots and their branches. Horizontal branching angles (or cardinal directions) cannot be specified as a parameter (such as parameter 3 in Table 3.1), which is a technical limitation of Rootbox. Measurements of averaged root diameter per distance interval are not affected by major 
differences in root direction. However, the inability to specify the horizontal angle of insertion limits practical control of model results, especially if the horizontal branching angle is known by practitioners in advance of modelling.

The use of five iterations for each scenario is meant to overcome the inherent variability of each Rootbox output, and follows the number of model iterations used in Dunbabin (2007), which averages the output of five model iterations to account for root architecture variability as a prerequisite for a sensitivity analysis. In future research, the number of iterations could be increased, but as of this writing there is no clear recommendation in the literature for an appropriate number of iterations to account for variability in the output of root architecture models.

\subsection{3 - Simulating Urban Conditions with Soil Grids}

In all scenarios, a basic soil grid was used which uniformly increases in value along the horizontal distance, and which decreases in value with depth, especially after $1 \mathrm{~m}$ depth. Both the tropism and elongation functions built into Rootbox allow for significant control of the output shape and vertical distribution of the roots, which in turn contributes to the practical reproduction of realistic root system profiles. All root types were programmed to react to this grid, except for the first two orders of the tap-root (i.e. root type 11 and 12; see Appendix A). This kept most of the resulting roots in the top $0.5 \mathrm{~m}$ of soil. Additionally, the elongation function reacted with low soil grid values below $0.5 \mathrm{~m}$, which reduced extension of root tips that penetrated these depths, so that in any simulation, the clear majority of roots stop growing if they grow deeper than $1 \mathrm{~m}$. Low grid values at greater depths simulate both a hypothetical water table, where roots typically do not penetrate or where they expire (Armstrong et al., 1976; Ballantyne, 1916; Coutts, 1987) and higher soil penetration resistance, which impedes root elongation and which is typically found at greater soil depths (Hernanz et al., 2002).

Some roots in urban (or constrained) growing conditions will penetrate deeper soil layers to exploit resources they might have otherwise acquired nearer to the surface (Day et al., 2010). Reproducing this growth propensity requires that grid values in specific locations deep under the surface should be higher than the surrounding values, creating a pocket of high tropism and low growth impedance. Scenario 2, which includes a localized nutrient or water source located 
between $0.2 \mathrm{~m}$ and $1 \mathrm{~m}$ under the surface does entice roots to penetrate greater depths. Roots are permitted to grow "through" to these depths because soil grid values do not trigger restrictions on root extension.

In reality, the reasons why a root system will orient towards a nutrient or water source are complex, and the ultimate path that the root takes is the result of multiple competing factors that both encourage and discourage directional growth. The growth direction that results is a trade-off between these factors (Mulia et al., 2010; Walk et al., 2006). The soil grid in Scenario 2 does influence growth direction, but a more complex tropism or elongation algorithm would have to be designed to adequately model the various ecological controls on directional growth. Designing these algorithms is beyond the scope of the present study, but should be considered in future research.

In Scenario 3, very low soil grid values, which represent a hypothetical sidewalk (concrete barrier) and a highly-compacted underlying region of soil, act as effective barriers to root elongation. In this scenario, several large roots grow under the area of impedance and then emerge towards the surface on the far side. In reality, highly compacted soil tends to limit root extension, and so the model output in this case is realistic, although actual roots react to a compaction impedance in various ways, and some roots may even increase their growth rate through compacted soil (Alameda et al., 2009; Coutts, 1987).

The base soil grid used in Scenario 1 is weighted to create a global directional growth bias towards 2 locations in Scenario 4. Locations of growth bias are placed at soil depths of 0.4 and $0.5 \mathrm{~m}$. These locations are meant to represent site locations where the existence of roots has been confirmed. In this way, Scenario 4 tests the ability of Rootbox's tropism function to optimize the spatial arrangement of roots to match existing site measurements. The weighting alters the tropism function by directing roots more towards these locations, but preserves the bias towards the periphery and upper soil layers. From the results, there is no discernable difference in the direction towards or presence of roots near these two locations. The reason why the weighting fails is likely because it amplifies the original directional bias towards the upper soil surface introduced in Scenario 1, despite slightly higher values found at the 2 locations. For comparison, Scenario 2 successfully attracts roots into deeper areas around the nutrient/water source, but the 
grid in this case is weighted locally, so that the tropism signal is comparable to that of the upper soil layers.

The results of these scenarios indicates that Rootbox is a potentially useful tool for simulating urban tree root systems in several ways. First, Rootbox allows root system architecture to exhibit generalized spatial attributes common to many tree species, such as a ZRT and heart root system shapes. This enables testing on a variety of site types because there is potential to capture the root growth habits of a variety of tree species. Second, the specification of a soil grid allows for the approximation of an urban soil condition. Third, tropism and elongation functions, coupled with a soil grid, allow users to specify and simulate both growth impedance and growth directional biases to better simulate root system responses to urban soil conditions. The value of Rootbox is that it offers a framework from which urban tree root models can be further developed, refined and implemented.

A major limitation of this research is that, while simulated tree root architecture is assessed to discern that it reproduces generalized morphological characteristics that would be expected to be found in many tree root systems (e.g. ZRT), this does not alone establish that the model can accurately predict the root architecture of existing site-specific tree root systems. While this research attempts to develop an approach to incorporating site-specific knowledge of existing root locations and site soil characteristics, by creating virtualized soil environments or, as in the case of scenario 4, the location of (hypothetically) known roots, it would be useful in future research to develop a method of constraining the output root architecture to more directly align with field-derived data. Such data could include root location, the amount, and location of, root branching; and root size and root order. This would build on the model's ability to reproduce a qualititatively realistic and generalized tree root architecture or response to hypothetical site conditions. In future work, the underlying functions of the Rootbox model should be modified to use data to control the expression of the root architecture production rules to ensure that model output conforms with empirical observations.

Another major limitation of this research is that it does not include a sensitivity analysis to determine the relative effect of changes in each parameter on specific outcomes. Thus, it is still uncertain whether some parameters, such as the strength of the tropism response, result in highly unrealistic architectural morphologies or spatial distributions. Sensitivity analysis of this kind is 
recommended to quantify the contribution of parameters to model output (Dunbabin et al., 2013; Morris, 1991; Pianosi et al., 2016). Sensitivity analyses have been used to evaluate parameter importance in root architecture models (Dunbabin, 2007; Garré et al., 2012), and it has been used as the basis for optimizing a root architectural model to produce specific outcomes given a certain site condition (Ma et al., 2008; Stava et al., 2014). The scope of the current project does not involve a sensitivity analysis primarily because of time resources, and sensitivity analyses typically include hundreds to thousands of model iterations to produce effective insights (Pianosi et al., 2016; Saltelli, et al., 2010). On the other hand, an architectural model need not be optimized to produce specific outcomes to be useful to interested practitioners. Part of the value of architectural models is in their ability to heuristically create mock-up scenarios that can be tested against data from direct site investigations or expert opinion, rather than to be predictive models (Dunbabin et al., 2013). There is usually a lack of data about tree root architecture and soil ecological conditions in urban contexts, and so calibrating a model's parameters to a general understanding about how urban conditions impact root architecture may appear pre-mature. Nonetheless, a sensitivity analysis would allow the greater user control over output, which is potentially valuable when porting the model between use in relation to diverse planting sites.

In the case where a root architecture model is used to predict, for example, the locations of roots, care would need to be taken to determine at which threshold and on what measure the model is considered accurate. If the root architecture model is being used to simulate the location of significant root density, then model performance likely would be best assessed based on comparison with root density measures from site observations, and not based on other measures, such as the precise location of individual roots. It is unlikely that any simulation, but especially a root architecture simulation, will be able to perfectly match observations in any instance, but future research should begin to develop a methodology that would determine a suitable indicator of accuracy, and at what threshold model accuracy is achieved..

This research does not consider small spatial or temporal resolution in root system development. Here, Rootbox is used unconventionally to simplify the complex functional-structural histories that result in specific root architectures. Fine roots, through which most soil-root interaction takes place (Coutts, 1987; Gregory, 2006) have lifespans that are very short compared to the lifetime of the root system of a tree, although it is at finer resolution that the architecture of the 
coarser, woody root system is ultimately determined. Only coarser woody roots are simulated within the current study. Soil grids used in each scenario describe conditions that are likely to persist through time, and assumes that they exert a constant influence throughout the "life" of the tree and influence the long-term architectural patterns. In this research, architecture at the level of fine roots was omitted firstly to reduce the complexity of the model inputs and outputs, and secondly to simplify the analysis of the resulting architecture.

Additionally, this research does not consider the impact of root thickening, nor root age and turnover, on the development of the tree root system. Root turnover, especially at the resolution of finer roots, has a subtractive effect on the number and distribution of roots in the system. In the demonstrated approach here, root number is set to reflect the values reported in tree excavation studies, which presumably already account for root turnover. In the current design of Rootbox, root age and turnover are controllable parameters, but these are not modulated by interaction with a soil environment. Root thickening is not built into the current software version. Further development of the Rootbox software should explore methods of integrating new functions that enable a soil model to moderate root age and death, and root thickening over time, in much the same way as soil models can moderate root growth direction and extention.

For urban forestry practitioners and landscape designers, modelling tree roots at the resolution of fine, absorbing roots, and incorporating root turnover functions, is likely to require significant time and computational resources, but can provide insight, especially where a history of a planting site is well known or where fine root architecture field measurements have been made on reference species to enable sufficient estimation of the relevant growth parameters.

Rootbox is built to be a general-purpose root architecture model that can be adapted to different plant contexts (Dunbabin et al., 2013) and can be retrofitted with custom functions as needed. Naturally, this requires programming skill on the part of users who want to apply the model to simulate tree root architecture in urban sites. The software implementation of Rootbox is written in MATLAB script, which has a well-developed documentation and a mature user base. In this project, several code additions had to be made to the original Rootbox code-base to allow for the creation of custom soil grids, which required familiarity with several advanced MATLAB functions. It is expected that future studies that improve upon outputs reported herein will require continued development in the MATLAB framework. 


\section{5 - Conclusions}

This project provides a demonstration of the use of Rootbox, a plant root architecture simulation program, to model the root architecture of urban trees in the context of four scenarios that represent hypothetical urban conditions. The study's results suggest that, through setting model parameters guided by reference to prevailing literature, Rootbox can generate an architecturally realistic tree root system that exhibits formal characteristics common to tree root systems reported in the literature. Rootbox's directional and elongation functions are used in conjunction with a soil model to simulate responses to hypothetical growth constraints commonly found in urban soils (i.e. growth attractions to certain locations and soil impedance caused by compaction and debris). The study demonstrates that Rootbox can be successfully adapted to modelling urban tree root architecture in various hypothetical settings and provides a flexible modelling framework that can be adapted to future, more sophisticated studies. However, the use of a threedimensional grid of encoded values to model a soil environment, coupled with select growth parameters and functions, needs to be approached with care to produce realistic and predictable results. Additionally, uncertainties remain regarding how changes in model parameterization affects changes in the model's output architecture. A sensitivity analysis, which would provide insight into how specific parameters are related to certain model outcomes, is a recommended next step in research seeking to optimize parameter selection to produce realistic tree root architectures that have predictive power on real sites. Moreover, further improvements to the model software can be made to incorporate fine root architecture as well as root diameter thickening and age parameters that respond to, and are constrained by, an input soil grid.

Roots are important biological components of trees, and architectural models like Rootbox are a potentially valuable addition to the repertoire of tools and methodological approaches available to urban forestry practitioners - arborists, engineers, and landscape and urban planners - for better understanding of how tree root systems may be explicitly arranged under ground in existing and hypothetical settings. While they remain the only ways to empirically investigate real urban tree roots, on-site field investigations that excavate or remotely detect tree roots using methods like ground penetrating radar (GPR), can be destructive and/or can be costly in both time and resources. Tree root architecture modelling can provide a hypothetical "mock up" of a tree root system that is driven by current understanding of how such systems develop 
underground. Opportunities exist to extend the predictive capabilities of tree root architecture simulations. This can not only aid in effective field investigations of tree root systems on existing sites, but can also aid in both their successful protection and management, and to inform the site design of new tree installations. 


\section{Chapter 4 - Limitations, and Opportunities for Further Research}

\section{1 - Research Limitations}

This research has several methodological limitations that include: (1) a lack of a methodology or approach for making Rootbox more predictive to align with empirical data derived from field observations and knowledge; (2) a need for a sensitivity and optimization analysis to refine Rootbox parameter selection to achieve more predictable, controlled model outcomes; and (3) a need for implementation of the Rootbox model at a finer spatial and temporal resolution to support its development into a more sophisticated functional-structural model, which can explicitly and comprehensively integrate the biological and ecological contexts of tree roots. The limitations of this research are similarly rooted in Rootbox's limited adaptability where creation of user-developed extensions to achieve new research and practice outcomes are challenging. Limitations are opportunities for further research and development of tree root architecture models to aid the work of interested users. In the context of current plant-modelling research, the development of comprehensive and fully-fledged functional-structural models of plant root systems, is a significant goal and an active area of development. However, in the case of Rootbox, upgrades and extensions to its model software or a combination of Rootbox with another tool, would likely be needed to support implementation of an advanced functionalstructural model of, especially, tree roots. In the case of urban growing conditions, such upgrades would likely require a fuller understanding of how tree roots interact with their soil environments over time, and this understanding is generally limited in the context of urban growing environments.

Both the limitations and opportunities of the current research may have different significance for either plant root researchers or practitioners, like arborists and landscape architects. For plantroot researchers, the current research is relevant because it documents a preliminary methodological approach to adapt Rootbox to the modelling of tree root architecture, which is not strongly considered in current plant-root architecture research (for a strong counter-example, see Mulia et al. (2010), discussed below). For practitioners, this research demonstrates the use of Rootbox to model tree root architecture on hypothetical urban sites. In turn, using Rootbox may provide input to site design and selection for new tree installations, and to inform planned site 
works to protect tree roots from damage. Additionally, Rootbox can aid on-site detection of existing tree roots by providing at least a preliminary way to integrate site-measurements such as the diameter of the tree, tree root system shape and likely maximal horizontal root system spread and individual root length, into the parameterization of the model. While Rootbox (and tree root modelling in general) is unlikely to be a complete substitute for site detection, especially on urban sites, its ability to generate virtual and hypothetical tree root architecture, based on measurements gained in the field or in relevant literature, make it a potential complement to site detection.

The following section describes three main limitations of the current research and its implementation of Rootbox. The limitations are followed with a discussion of the possible avenues for further research that arise from these limitations.

\subsection{1 - Rootbox as a Predictive Model of Actual Tree Root Systems}

While Rootbox can simulate generalized morphological characteristics of tree root architecture

(e.g. ZRT), and each output qualititatively appears to be a plausible rendition of what root system looks like, Rootbox does not currently have the ability to predict the precise locations or other attributes of any actual individual site-specific tree root system. While this research attempts to develop an approach to incorporating site-specific knowledge of existing root locations and site soil characteristics, by creating virtualized soil environments or, as in the case of scenario 4, the location of (hypothetically) known roots, it would be useful in future research to develop a method of constraining the output root architecture to more directly align with field-derived data. Rootbox could be further developed into a predictive model, and could potentially overcome the difficulties encountered in the attempt, as detailed in Scenario 4, to constrain the occurrence of roots so that they are in agreement with empirically-determined locations. There is as yet no clear methodological approach to incorporating site data to work with Rootbox growth functions. However, the relationship between tropism values in the soil grid and root occurrence at specific locations could be determined, for example, to optimize the selection of soil grid values. In Scenario 4, there is substantial uncertainty about how the soil grid values should be weighted to achieve a desired output. 
Alternatively, a more fruitful approach to making Rootbox more predictive of empiricallydetermined root locations might include the redesign of underlying growth functions to better "lead" roots through pre-defined spatial areas. It is unclear whether a tropism function, which influences the direction of root growth, is sufficiently effective to guarantee the root system occurrence at pre-defined locations. On the other hand, it might also be helpful to take the density-based approach as described in Mulia et al. (2010), which avoids the need to constrain growth functions and instead models the location(s) of roots (and the plant root architecture) more directly as its own three-dimensional grid.

Assessing the accuracy (or success) of Rootbox's output must clarify which aspect of the output is relevant. Rootbox may, for example, always be able to reproduce more generalized characteristics, such as the ZRT or the maximum horizontal root spread, quite accurately in comparison to empirically-derived values. However, Rootbox may not be able to accurately reproduce more detailed characteristics, such as a precise match between simulated and observed root location. It is unlikely that any simulation, but especially a root architecture simulation, will be able to perfectly match observations in any instance, but future research should begin to develop a methodology that would determine a suitable indicator of accuracy, and at what threshold model accuracy is achieved.

\subsection{2 - Sensitivity Analysis and Model Parameter Optimization}

In this research, Rootbox was parameterized manually using references to literature that describe the architecture of tree root systems in several field conditions. Model parameterization based on literature describing plant root architecture is a valid approach when there are no other options available, as demonstrated in Leitner et al. (2010a; 2010b) and in Dunbabin (2007). In this research, there was no field-derived tree root architectural data that could be used for parameterization. Crucially, even if such field-derived data is available, it is typically difficult to predict how specific parameter values (or combinations of such values) will work to produce a specific output root architecture. In this research, model parameterization was conducted by trial and error to eliminate obviously unrealistic outputs (see the Methods section in Chapter 3). Setting length-, thickness-, and direction-controlling parameters was established iteratively by testing different configuration settings and assessing the output visually. While the iterative 
approach is to some extent advantageous because it allows for heuristic experimentation (Dunbabin, et al., 2013; Fourcaud et al., 2008), this approach takes time and makes the model highly dependent on assumptions about how alternative parameterizations will alter model outcomes. In practice, it is useful for the model to be applicable to a variety of different growth contexts (urban or otherwise) but at the same time it is useful to understand what parametrizations schemes have the most utility for model implementation and are the most likely to result in realistic, desired outcomes.

A sensitivity analysis could be used to make parameterization of Rootbox more efficient and to reduce uncertainties involved in the iterative approach. Similarly, such an analysis allows parameters to be ranked in importance and to quantify the effect of changes in their values on the model's output. Dunbabin (2007) used a modified Elementary Effects method (detailed in Morris, 1991) to quantify the effects of making small changes in several input parameters of the ROOTMAP plant root architecture model on defined model outcomes, such as the effects of weed competition. Use of this method allows for statistical relationships to be established between individual parameters and changes in their values. When the relationship is established, parameter values can be linked to their respective outcomes, but the inverse is possible as well: outcomes, or even particular field measurements, can be linked back to specific parameter settings, which may allow practitioners to establish a stronger link between their field measurements and the tree root architecture models. Linking in this way provides a method of optimizing parameter values, as is demonstrated in Ma et al. (2008), who use a generalized least squares approach to linking parameters and outcomes of a maize plant architecture model (above-ground components only). These authors present their approach as a calibration method with wide applicability to different growth contexts. A similar sensitivity analysis of Rootbox parameters, irrespective of the detailed methodology (i.e. whether the study uses elementary effects or a regression) could yield practice "guidelines" for which parameter configurations are ideal given a set of field conditions measured in advance.

A sensitivity analysis was not conducted as a part of this research due mainly to the time and computation limits involved. To conduct a meaningful analysis, a model needs to be run many times (Pianosi et al. 2016). In Dunbabin (2007), the ROOTMAP model was run over 2400 times; for regression-based methods like the kind detailed in Ma et al. (2008), the number of model 
iterations is potentially much higher (Pianosi et al. 2016). However, this limitation may not be encountered in future research efforts that focus on performing a sensitivity analysis for parameterization of Rootbox in urban settings, especially if computational resource limits can be overcome.

Greater user control over model outputs would allow practitioners to incorporate Rootbox into their field-work by enabling them to use - at the very least - empirical root location measurements as an input to the model. Whether this control is achieved either through a sensitivity analysis or new dynamic growth functions that use input data to affect the spatial arrangements of the output root architecture, is a user interface design question that should be undertaken in future research. Any software design should take into consideration the expertise of the user; Rootbox as currently designed includes only some design features that make it userfriendly, and most advanced functions, including modelling a large amount of root types, requires some programming skills in MATLAB.

\subsection{3 - Incorporation of fine roots, root thickening and root turnover}

In this research, Rootbox was used to simulate a system of coarse tree roots without consideration of fine roots, even though the contribution of fine roots to the development of tree root system architecture is ecologically significant. Fine roots are responsible for all the plant's resource acquisition from soil, and they play a large part in how the tree root system tends to spatially distribute over time. Thus, models that consider root-soil interactions on the spatial scale of fine roots are potentially more robust in their ability to simulate the actual biological controls at play in a growth site that impact long term tree root architecture.

In this demonstration of Rootbox, only coarse roots were included because: (1) it is computationally onerous to model the architecture of fine roots explicitly; and (2) since Rootbox does not model root thickening over time, it is difficult to code production rules that simulate "secondary thickening" (i.e. the process of conversion of fine roots to perennial woody roots).

One way to solve the problem of computational complexity is to incorporate fine roots as a "scaled up", or aggregated component within the model, as described in Mulia et al. (2010). Interestingly, these authors develop their model to aggregate the architecture of the entire root 
system of two trees into a three-dimensional grid of voxels (i.e. three-dimensional volume units that store attributes). While they develop a functional-structural model which takes account of both fine and coarse roots on a multi-year scale, the model does not consider the trees' root architecture explicitly and the architecture must be extrapolated by additional functions within their software framework. However, an opportunity for further research could use Mulia et al.'s (2010) approach as a precedent for integrating novel root growth functions within the MATLAB framework, especially when implementing custom soil grids alongside core Rootbox functions.

Rootbox includes options for varying growth parameters through time, including root turnover and death, but in the current demonstration growth parameters are kept constant, roots elongate until they reach their parameterized elongation limits and root death is not considered. Instead, Rootbox, in this case, is used to create an "idealized" root architecture (such as that considered in Nygren et al. (2009)) that responds to parameters and soil growth biases that do not change through time. This approach was taken to simplify model implementation, and to avoid complexities like those involved with including fine roots. Rootbox includes only one root-age controlling parameter, which is a value that is not modulated by a soil model. While this parameter could have been used to simulate the "thinning" of the root system over time, the implementation in this research sought only to estimate root number and spread using values from the literature that describes one-time tree excavations. It is assumed that Rootbox users would implement it in the same way, unless there was a need to consider the rate of coarse root turnover. Future research could improve Rootbox by designing and writing a new function (much like the tropism or elongation functions) that make root death dependent on interactions with a soil grid. For instance, values for concentrations of water or nutrient could be encoded in a custom soil grid, and could trigger root stress that, after a certain amount of simulation time, would cause root death.

In addition to root death over time, root thickening as a function of age and growth impedance (Coutts, 1987) could be incorporated into the model through re-design of the software components that govern root diameter over the course of the simulation. Root thickening, like root age, cannot be modified by a soil grid in the current iteration of Rootbox, yet characteristics of the soil environment that tend to impede root growth, such as soil compaction or soil debris, are likely to be the variables that modify local changes in root diameter over time. 
The inclusion of both fine roots and changes in root growth, age and diameter over time, would not alone create a sophisticated functional-structural model of tree root systems, but would be early steps toward allowing Rootbox to consider more sophisticated biological and ecological processes that may be of use to practitioners who wish to incorporate these factors in their work. Potentially, this consideration may necessitate a substantial re-design of the Rootbox framework, especially if "scale up" approaches, such as those in Mulia et al.(2010) are pursued. Additionally, if any future studies modify the Rootbox software to perform different functions, programming expertise in MATLAB would be required to achieve most upgrades and extensions. An advantage to using Rootbox is that it is open-source and the MATLAB framework is welldocumented. While this enables Rootbox to be applied in a variety of research and practice settings, it also makes upgrade or re-design by programmers easier than it may be for inflexible and highly-specialized software designs. 
Appendix A - selected Rootbox parameters

\begin{tabular}{|c|c|c|c|c|c|c|c|c|c|c|c|c|c|c|c|c|c|c|c|}
\hline \# & $\begin{array}{l}\text { Parameter } \\
\text { Name }\end{array}$ & Unit & 1 & 2 & 3 & 4 & 5 & 6 & 7 & 8 & 9 & 10 & 11 & 12 & 13 & 14 & 15 & 16 & 17 \\
\hline 1 & $\begin{array}{l}\text { Elongation } \\
\text { rate* }^{*}\end{array}$ & $\mathrm{~cm}$ & $\begin{array}{l}10 \\
(0)\end{array}$ & $\begin{array}{l}10 \\
(0)\end{array}$ & $\begin{array}{l}10 \\
(0)\end{array}$ & $10(0)$ & $\begin{array}{l}10 \\
(0)\end{array}$ & $\begin{array}{l}10 \\
(0)\end{array}$ & $10(0)$ & $10(0)$ & $\begin{array}{l}10 \\
(0)\end{array}$ & $\begin{array}{l}10 \\
(0)\end{array}$ & $\begin{array}{l}10 \\
(0)\end{array}$ & $\begin{array}{l}10 \\
(0)\end{array}$ & $\begin{array}{l}10 \\
(0)\end{array}$ & $10(0)$ & $10(0)$ & $10(0)$ & $10(0)$ \\
\hline 2 & Root radius* & $\mathrm{cm}$ & $3(0)$ & $\begin{array}{l}2.5 \\
(0)\end{array}$ & $\begin{array}{c}1 \\
(0.2)\end{array}$ & $\begin{array}{c}0.8 \\
(0.04)\end{array}$ & $\begin{array}{c}0.7 \\
(0.0 \\
4)\end{array}$ & $\begin{array}{l}0.6 \\
(0.0 \\
4)\end{array}$ & $\begin{array}{c}0.3 \\
(0.08)\end{array}$ & $\begin{array}{c}0.15 \\
(0.08)\end{array}$ & $\begin{array}{l}0.15 \\
(0)\end{array}$ & $\begin{array}{l}0.10 \\
(0)\end{array}$ & $3(0)$ & $\begin{array}{l}2.5 \\
(0)\end{array}$ & $\begin{array}{c}1 \\
(0.2)\end{array}$ & $\begin{array}{c}0.3 \\
(0.08)\end{array}$ & $\begin{array}{c}0.7 \\
(0.04)\end{array}$ & $\begin{array}{c}0.6 \\
(0.04)\end{array}$ & $\begin{array}{c}0.3 \\
(0.08)\end{array}$ \\
\hline 3 & $\begin{array}{l}\text { Insertion } \\
\text { angle* }^{*}\end{array}$ & degrees & $\begin{array}{l}90 \\
(0)\end{array}$ & $0(0)$ & $5(0)$ & $5(0)$ & $\begin{array}{l}15 \\
(0)\end{array}$ & $\begin{array}{l}15 \\
(0)\end{array}$ & $85(0)$ & $5(0)$ & $\begin{array}{l}45 \\
(0)\end{array}$ & $\begin{array}{l}90 \\
(0)\end{array}$ & $0(0)$ & $\begin{array}{l}20 \\
(0)\end{array}$ & $5(0)$ & $45(0)$ & $45(0)$ & $45(0)$ & $85(0)$ \\
\hline 4 & Basal zone * & $\mathrm{cm}$ & $5(0)$ & $\begin{array}{l}10 \\
(0)\end{array}$ & $\begin{array}{l}30 \\
(0)\end{array}$ & $50(0)$ & $5(0)$ & $\begin{array}{l}10 \\
(0)\end{array}$ & $\begin{array}{c}200 \\
(0)\end{array}$ & $10(0)$ & $5(0)$ & $0(0)$ & $1(0)$ & $5(0)$ & $5(0)$ & $5(0)$ & $5(0)$ & $5(0)$ & $10(0)$ \\
\hline 5 & $\underset{*}{\text { Apical zone }}$ & $\mathrm{cm}$ & $5(0)$ & $\begin{array}{l}24 \\
(0)\end{array}$ & $6(0)$ & $50(0)$ & $\begin{array}{c}250 \\
(0)\end{array}$ & $\begin{array}{l}100 \\
(0)\end{array}$ & $5(0)$ & $\begin{array}{l}200 \\
(0)\end{array}$ & $\begin{array}{l}100 \\
(0)\end{array}$ & $\begin{array}{l}40 \\
(0)\end{array}$ & $\begin{array}{l}10 \\
(0)\end{array}$ & $\begin{array}{l}20 \\
(0)\end{array}$ & $\begin{array}{l}40 \\
(0)\end{array}$ & $\begin{array}{c}200 \\
(0)\end{array}$ & $50(0)$ & $\begin{array}{c}200 \\
(0)\end{array}$ & $200(0)$ \\
\hline 6 & $\begin{array}{l}\text { Distance } \\
\text { between } \\
\text { each } \\
\text { successor* }\end{array}$ & $\mathrm{cm}$ & $2(0)$ & $3(0)$ & $2(0)$ & $14(0)$ & $\begin{array}{l}50 \\
(0)\end{array}$ & $\begin{array}{c}110 \\
(0)\end{array}$ & $50(0)$ & $35(0)$ & $\begin{array}{l}10 \\
(0)\end{array}$ & $N / A$ & $5(0)$ & $4(0)$ & $2(0)$ & $N / A$ & $7(0)$ & $39(0)$ & $N / A$ \\
\hline 7 & $\begin{array}{l}\text { Root type of } \\
\text { successor }\end{array}$ & $\mathrm{cm}$ & 2 & 3 & 4 & 5 & 6 & 7 & 8 & 9 & 10 & $N / A$ & 12 & 13 & 14 & $N / A$ & 16 & 17 & $N / A$ \\
\hline 8 & $\begin{array}{l}\text { Maximum } \\
\text { number of } \\
\text { successors }\end{array}$ & $\mathrm{cm}$ & $1(0)$ & $2(0)$ & $1(0)$ & $3(0)$ & $1(0)$ & $1(0)$ & $1(0)$ & $10(0)$ & $2(0)$ & $N / A$ & $2(0)$ & $2(0)$ & $5(0)$ & $N / A$ & $5(0)$ & $5(0)$ & $N / A$ \\
\hline 9 & $\begin{array}{l}\text { Spatial } \\
\text { resolution } \\
\text { along the } \\
\text { axis of the } \\
\text { root }\end{array}$ & $\mathrm{cm}$ & 1 & 1 & 1 & 1 & 1 & 1 & 1 & 1 & 1 & 1 & 1 & 1 & 1 & 1 & 1 & 1 & 1 \\
\hline 10 & $\begin{array}{l}\text { Segment } \\
\text { flexibility }\end{array}$ & None & 0.05 & 0.05 & 0.05 & 0.05 & 0.05 & 0.05 & 0.05 & 0.05 & 0.05 & 0.05 & 0.05 & 0.1 & 0.1 & 0.1 & 0.05 & 0.05 & 0.05 \\
\hline 11 & $\begin{array}{l}\text { Tropism } \\
\text { strength }\end{array}$ & None & 0.1 & 0.1 & 0.5 & 0.3 & 0.25 & 0.5 & 0.5 & 0.5 & 0.25 & 0.25 & 0.1 & 0.1 & 0.1 & 0.25 & 0.25 & 0.5 & 0.5 \\
\hline 12 & $\begin{array}{l}\text { Tropism } \\
\text { type }\end{array}$ & None & Soil & Soil & Soil & Soil & Soil & Soil & Soil & Soil & Soil & Grav & Grav & Grav & Grav & Soil & Soil & Soil & Plagio \\
\hline 13 & $\begin{array}{l}\text { Maximum } \\
\text { root } \\
\text { lifespan* }\end{array}$ & $\begin{array}{l}\text { Time } \\
\text { unit }\end{array}$ & $N / A$ & $N / A$ & $N / A$ & $N / A$ & $N / A$ & $N / A$ & $N / A$ & $N / A$ & $N / A$ & $N / A$ & $N / A$ & $N / A$ & $N / A$ & $N / A$ & $N / A$ & $N / A$ & $N / A$ \\
\hline 14 & $\begin{array}{l}\text { Scale } \\
\text { elongation }\end{array}$ & None & No & No & Yes & Yes & Yes & Yes & Yes & Yes & Yes & Yes & Yes & Yes & Yes & Yes & Yes & Yes & Yes \\
\hline 15 & $\begin{array}{l}\text { Scale } \\
\text { successor } \\
\text { probability }\end{array}$ & None & No & No & No & No & No & No & No & No & No & No & No & No & No & No & No & No & No \\
\hline 16 & $\begin{array}{l}\text { Scale } \\
\text { insertion } \\
\text { angle }\end{array}$ & None & No & No & No & No & No & No & No & No & No & No & No & No & No & No & No & No & No \\
\hline
\end{tabular}


Appendix A provides a list of the Rootbox growth parameter values used in each simulation for each root type (top header). This list corresponds with the parameter description given in Table 3.1 in Chapter 3.2, Methods. All parameter names marked with an asterisk (*) may vary stochastically within a standard deviation, which are values indicated in brackets after the parameter value. If there is a (0) standard deviation, then this value does not vary during the simulation. If there is a standard deviation, then the parameter value is the mean around which the values vary.

Tropism type (parameter 12) values are given as shorthands:

Soil $=$ growth directional bias controlled by the custom soil grid.

Grav = gravitropism, where directional bias is determined by favouring absolute growth angles that tend downwards (i.e. vertical growth).

Plagio $=$ plagiotropism, where directional bias is determined by favouring absolute growth angles of 0 degrees (i.e. horizontal growth). 

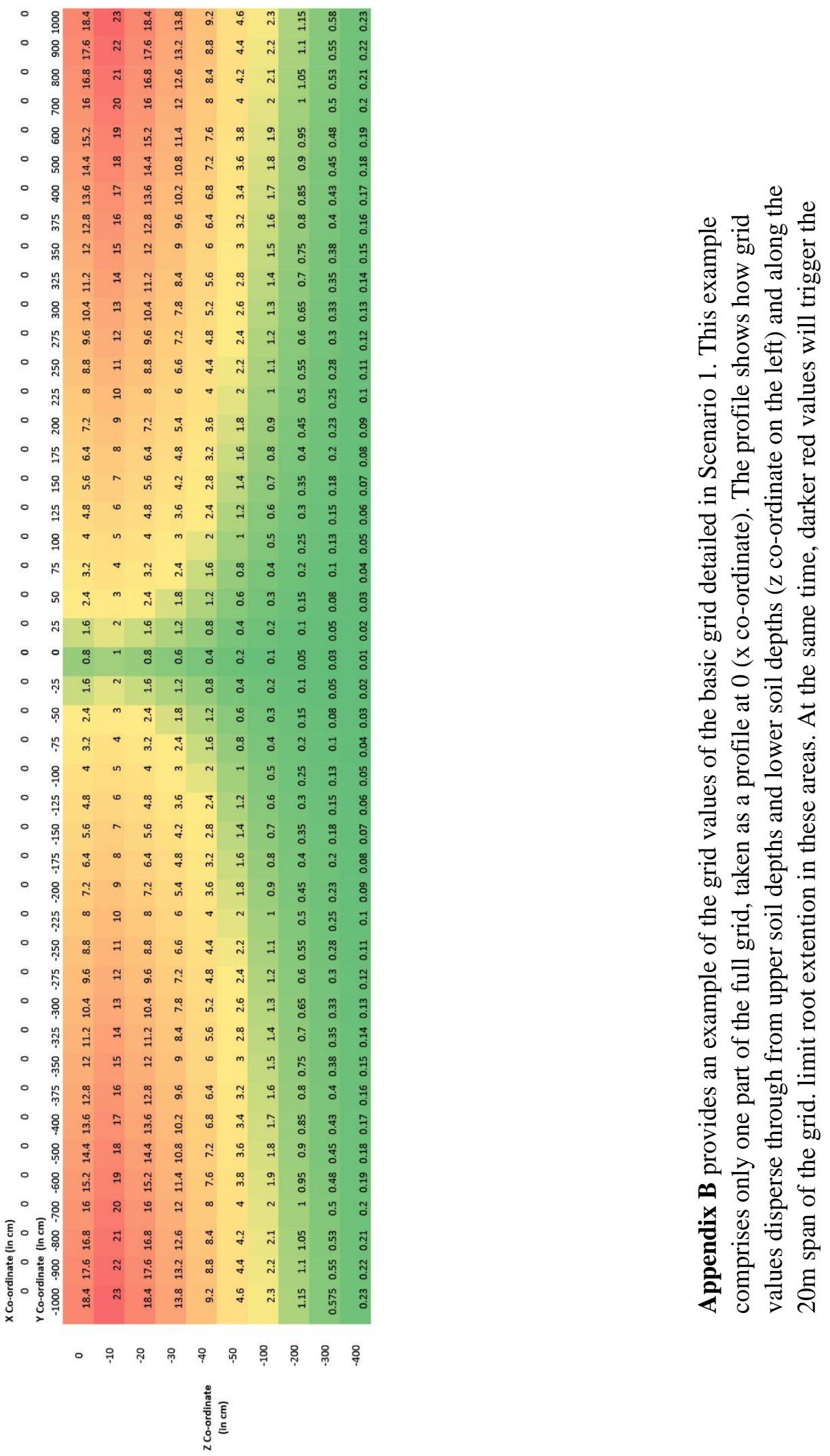


\section{Appendix C}

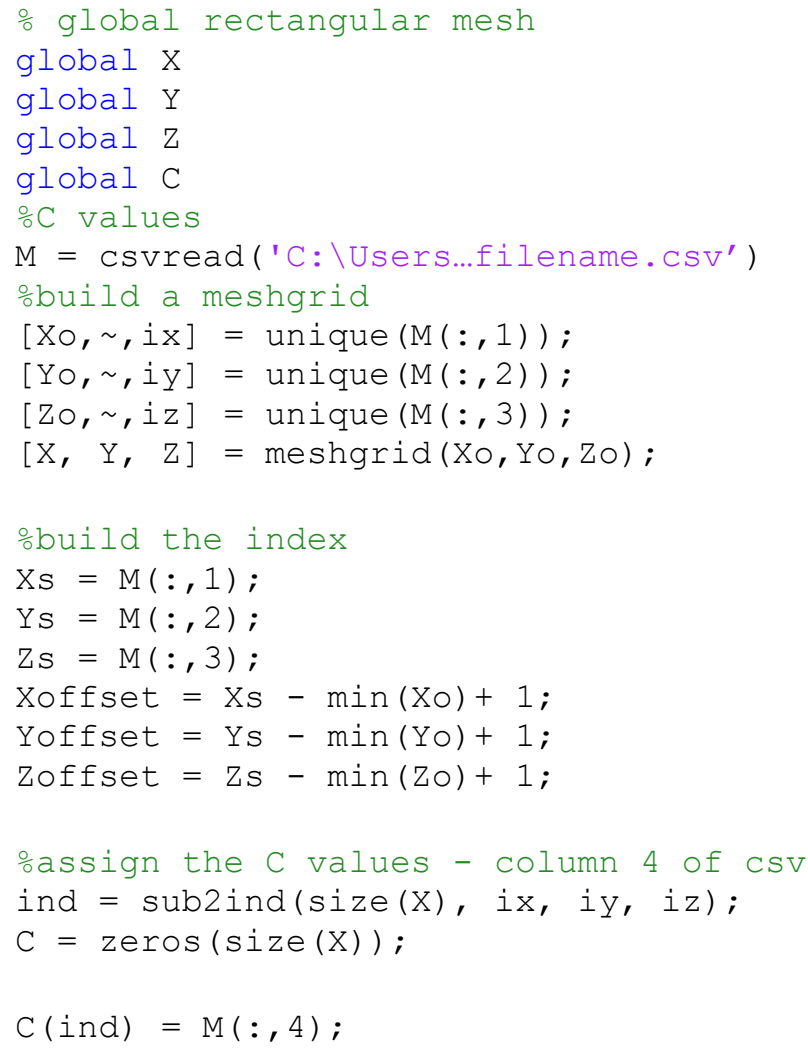

Appendix C provides an example of a MATLAB script that reads an input csv that specifies a soil environment and turns it into a MATLAB-based meshgrid. The csv file must contain no more than 4 columns. The first three columns specify the $\mathrm{x}, \mathrm{y}$, and $\mathrm{z}$ spatial co-ordinates of the values and are used to assign values to locations in the grid. The fourth column specifies the attribute value (e.g. lower numbers indicating a growth impediment) that is then assigned these locations. 


\section{$\underline{\text { References }}$}

Alameda, D., \& Villar R. (2009). Moderate soil compaction: Implications on growth and architecture in seedlings of 17 woody plant species. Soil and Tillage Research, 103, 325331. doi - 10.1016/j.still.2008.10.029

Alberty, C., Pellett, H., \& Taylor, D. (1984). Characterization of Soil Compaction at Construction Sites and Woody Plant Response. J. Environ. Hort., 2(2), 48-53.

Amato, R., Basso, B., Bitella, G., Celano, G., \& Gianfranco, R. (2008). In situ detection of tree root distribution and biomass by multielectroderesistivity imaging. Tree Physiology, 28, 1441-1448. doi - 10.1093/treephys/28.10.1441

Ampoortera, E., De Frennea, P., Hermyb, M., \& Verheyena, K. (2011). Effects of soil compaction on growth and survival of tree saplings: A meta-analysis. Basic and Applied Ecology, 12, 394-402. doi - 10.1016/j.baae.2011.06.003

Ballantyne, A. (1916). "Bulletin No. 143 - Fruit Tree Root Systems: Spread and Depth". UAES Bulletins. Paper 109.

Blume, H.P. (1989) Classification of Soils in Urban Agglomerations. Catena, 16, 269-275. doi 10.1016/0341-8162(89)90013-1

Boswell, D., \& Foucher, T. (2011). The art of readable code. Sebastopol, California: O'Reilly.

Brunner, I., Arend, M., Dawes, M., Herzog, C., \& Sperisen, C. (2015). How Tree Roots Respond to Drought. Frontiers in Plant Science, 6 (547), 1-16. doi $10.3389 /$ fpls.2015.00547

Bühler, O., Ingerslev, M., Kristoffersen, P., Nielsen, C., Schou, E., Skov, S., \& Thomsen, I. (2017). Tree development in structural soil - an empirical below-ground in-situ study of urban trees in Copenhagen, Denmark. Plant Soil, 413, 29-44. doi 10.1007/s 11104-016-2814-4

Clausnitzer, V., \& Hopmans, J. (1994). Simultaneous modeling of transient three-dimensional root growth and soil water flow. Plant and Soil, 164, 299-314. doi 10.1007/BF00010082

Collet, C., Lof, M., Pagès, L. (2006). Root system development of oak seedlings analysed using an architectural model. Effects of competition with grass. Plant and Soil, 279, 367-383. doi - 10.1007/s 11104-005-2419-9

Coutts, M. P. (1989). Factors affecting the direction of growth of tree roots. Ann. Sci. For., 46, 277-287. doi 10.1051/forest:19890565

Coutts, M. P. (1987). Developmental processes in tree root systems. Canadian Journal of Forest Research ,17, 761-767. 
Coutts, M., P., \& Nicoll, B. (1991). Orientation of the Lateral Roots of Trees. I. Upward Growth of Surface Roots and Deflection Near the Soil Surface. New Phytologist 119, 227-234. doi - 10.1111/j.1469-8137.1991.tb01025.x

Couvreur, V., Javaux, M., \& Vanderborght, J. (2012). A simple three-dimensional macroscopic root water uptake model based on the hydraulic architecture approach. Hydrol. Earth Syst. Sci. 16, 2957-2971. doi - 10.5194/hess-16-2957-2012

Crow, P. (2005). The influence of soils and species on tree root depth. Information Note FCINO78 Forestry Commission Edinburgh. https://www.forestry.gov.uk/pdf/fcin078.pdf/\$file/fcin078.pdf

Danjon, F., Fourcaud, T., \& Reubens, B. (2005). Root architecture and wind-firmness of mature Pinus pinaster. New Phytologist, 168, 387-400. doi - 10.1111/j.1469-8137.2005.01497.x

Danjon, F., \& Reubens, B. (2008). Assessing and analyzing 3D architecture of woody root systems, a review of methods and applications in tree and soil stability, resource acquisition and allocation. Plant Soil, 303, 1-34. doi - 10.1007/s 11104-007-9470-7

Day, S., \& Wiseman, E. (2010). At the root of it. Arborist News, 20-22. url http://urbanforestry.frec.vt.edu/documents/articles/Roots1_Arb_News.pdf

Day, S., Dickinson S., Harris, J. \& Wiseman, E. (2010). Contemporary Concepts of Root System Architecture of Urban Trees. Arboriculture \& Urban Forestry, 36(4), 149-159.

Dunbabin, V., Chen, Y., Diggle, A, Javaux, M., Leitner, D., Pagès, L., Postma, J., Schnepf, A., Rengel, Z., \& Wu, L. (2013) Modelling root-soil interactions using three-dimensional models of root growth, architecture and function. Plant Soil, 372, 93-124. doi 10.1007/s11104-013-1769-y

Dunbabin, V. (2007). Simulating the role of rooting traits in crop-weed competition. Field Crops Research, 104, 44-51. doi - 10.1016/j.fcr.2007.03.014

Dupuy, L., Danjon. F., Fourcaud, T., \& Stokes, A. (2005). A density-based approach for the modelling of root architecture: application to Maritime pine (Pinus pinaster Ait.) root systems. Journal of Theoretical Biology 236, 323-334. doi- 10.1016/j.jtbi.2005.03.013

Fahey, T., \& Hughes, J. (1994). Fine Root Dynamics in a Northern Hardwood Forest Ecosystem, Hubbard Brook Experimental Forest, NH. Journal of Ecology, 82 (3), 533-548. doi $10.2307 / 2261262$

Firn, R., \& Digby, J. (1981). The establishment of tropic curvatures in plants. Annual Review of Plant Physiology, 31,131-148. doi - 10.1146/annure v.pp.31.060180.001023

Forde, B. (2009). Is it good noise? The role of developmental instability in the shaping of a root system. Journal of Experimental Botany, 60 (14), 3989-4002. doi - 10.1093/jxb/erp265

Forde, B., \& Lorenzo, H. (2001). The nutritional control of root development. Plant and Soil, 232, 51-68. doi - 10.1023/A:1010329902165 
Fourcaud, T., Korner, C., Lambers, H., Stokes, A., \& Zhang, X. (2008). Plant Growth Modelling and Applications: The Increasing Importance of Plant Architecture in Growth Models. Annals of Botany, 101, 1053-1063. doi - 10.1093/a ob/mcn050

Garré, S., Javaux, M., Laloy, E., Pagès, L., Vanderborght, J., \& Vereecken, H. (2012). Parameterizing a Dynamic Architectural Model of the Root System of Spring Barley from Minirhizotron Data. Vadose Zone Journal, 11(4), 1539-1663. doi - 10.2136/vzj2011.0179

Ge, Z., Lynch, J., \& Rubio, G. (2000). The importance of root gravitropism for inter-root competition and phosphorus acquisition efficiency: results from a geometric simulation model. Plant and Soil 218, 159-171. doi - 10.1023/A:1014987710937

Giuliani, F., Autelitano, F., Degiovanni, E., \& Montepara, A. (2015). DEM modelling analysis of tree root growth in street pavements. International Journal of Pavement Engineering, 18(1), 1-10. doi - 10.1080/10298436.2015.1019495

Godin, C., \& Sinoquet, H. (2005). Functional-structural plant modelling. New Phytologist, 166, 705-708. doi - 10.1111/j.1469-8137.2005.01445.x

Gregory, P. Plant Roots: Growth, Activity and Interaction with Soils. (2006). Oxford: Blackwell Publishing.

Guo, L., Chen, J., Cui, X., Fan, B., \& Lin, H. (2013). Application of ground penetrating radar for coarse root detection and quantification: a review. Plant and Soil, 362(1-2), 1-23. doi $10.1007 / \mathrm{s} 11104-012-1455-5$

Hawver, G., \& Bassuk, N. (2006). Soils: The Key to Successful Establishment of Urban Vegetation. In Kuser, J. (Ed.). Urban and Community Forestry in the Northeast. (pp. 165182). Netherlands.

Heinen, M., De Willigen, P., \& Mollier, A. (2003). Growth of a root system described as diffusion. II. Numerical model and application. Plant and Soil, 252, 251-265. doi 10.1023/A:1024749022761

Henderson, R., Ford, E., Renshaw, E., \& Deans, J. (1983). Morphology of the Structural Root System of Sitka Spruce 1. Analysis and Quantitative Description. Forestry, 56 (2), 121135. doi - 10.1093/fore stry/56.2.121

Hernanz, J., Peixoto, H., Cerisola, C., Sanchez-Giron, V. (2000). An empirical model to predict soil bulk density profiles in field conditions using penetration resistance, moisture content and soil depth. Journal of Terramechanics, 37, 167-184. doi - 10.1016/S00224898(99)00020-8

Jackson, R., Hoffmann, W., Linder, C., Moore, L., \& Pockman, W. (1999). Ecosystem rooting depth determined with caves and DNA. Proc. Natl. Acad. Sci., 96, 11387-11392. doi 10.1073/pnas.96.20.11387

Jourdan, C., \& Rey, H. (1997). Modelling and simulation of the architecture and development of the oil-palm (Elaeis guineensis Jacq.) root system. Plant and Soil, 190, 235-246. doi 10.1007/s 11104-006-9030-6 
Juniper, B. (1976). Geotropism. Annual Review of Plant Physiology, 27, 385-406. doi -10.1146/annurev.pp.27.060176.002125

Kalogiros, D., Adu, M., Bengough, A., Broadley, M., Dray, X., Dupuy, L., Ptashnyk, M., \& White, P. (2016). Analysis of root growth from a phenotyping data set using a densitybased model. Journal of Experimental Botany, 67(4), 1045-1058. doi - 10.1093/jxb/erv573

Leitner, D., Bodner, G., Klepsch, S., \& Schnepf, A. (2010a). A dynamic root system growth model based on L-systems. Plant and Soil, 332(1), 177-192. doi -10.1007/s 11104-010-02847

Leitner, D., Klepsch, S., Knieß, A., \& Schnepf, A. (2010b). The algorithmic beauty of plant roots - an L-System model for dynamic root growth simulation. Mathematical and Computer Modelling of Dynamical Systems, 16(6), 575-587. doi - 10.1080/13873954.2010.491360

Leitner, D., Klepsch, S., Roose, T., Schnepf, A. (2010c). Comparison of nutrient uptake between three-dimensional simulation and an averaged root system model. Plant Biosystems, 144(2), 443-447. doi - 10.1080/11263501003726334

Lenk, F., Bley, T., Oberthur, P., Schneider, M., Steingroewer, J., \& Surmann, A. (2014). Modeling hairy root tissue growth in in vitro environments using an agent-based, structured growth model. Bioprocess Biosyst Eng, 37, 1173-1184. doi 10.1007/s00449-013-1088-y

Lontoc-Roy, M., Brouillet, T., Dutilleul, P., Han, L., Prasher, S., \& Smith, D. (2006). Advances in the acquisition and analysis of CT scan data to isolate a croproot system from the soil medium and quantify root system complexity in 3-D space. Geoderma, 137, 231-241. doi - 10.1016/j.geoderma.2006.08.025

Lopez, L., Bais, H., Liu, L., Shantharaj, D., \& Yu, J. (2011). Robust image-based 3D Modeling of Root Architecture. Computer Graphics International.

Ma, Y., Courne, P., De Reffye, P., Guo, Y., Li, B, \& Wen, M. (2008). Parameter Optimization and Field Validation of the Functional-Structural Model GREENLAB for Maize at Different Population Densities. Annals of Botany, 101, 1185-1194. doi $10.1093 / \mathrm{aob} / \mathrm{mcm} 233$

Marks, C., \& Lechowicz, M. (2006). A holistic tree seedling model for the investigation of functional trait diversity. Ecological Modelling, 193, 141-181. doi 10.1016/j.e colmode1.2005.09.011

McIvor, I., Douglas, G., Hurst, S., Hussain, Z., \& Foote, A. (2008). Structural root growth of young Veronese poplars on erodible slopes in the southern North Island, New Zealand. Agroforest Syst, 72, 75-86. doi - 10.1007/s 10457-007-9090-5

Morris, M. (1991). Factorial Sampling Plans for Preliminary Computational Experiments. Technometrics, 33 (2), 161-174. doi - 10.1080/00401706.1991.10484804 
Mulia, R., Dupraz, C. \& van Noordwijk, M. (2010). Reconciling root plasticity and architectural ground rules in tree root growth models with voxel automata. Plant Soil, 337, 77-92. doi 10.1007/s 11104-010-0502-3

Murray, R. (2006). Tree Biology Notebook. (2nd ed. 2011). Maryland, USA: STL Publishing.

Nicoll, B., \& Armstrong, A. (1998). Development of Prunus Root Systems in A City Street: Pavement Damage and Root Architecture. Arboricultural Journal, 22, 259-270. doi 10.1080/03071375.1998.9747209

Nygren, P., Miaoer, L., \& Ozier-Lafontaine, H. (2009). Effects of turnover and internal variability of tree root systems on modelling coarse root architecture: comparing simulations for young Populus deltoides with field data. Canadian Journal of Forest Research, 39, 97-108. doi - 10.1139/X08-158

Page, J., Hunt, R., \& Winston, W. (2015). Soils beneath suspended pavements: An opportunity for stormwater control and treatment. Ecological Engineering, 82, 40-48. doi 10.1016/j.e coleng.2015.04.060

Pagès, L., Bécela, C., Boukcimb, H., Moreauc, D., Nguyend, C., \& Voisin, A. (2014). Calibration and evaluation of ArchiSimple, a simple model of rootsystem architecture. Ecological Modelling 290, 76-84. doi - 10.1016/j.e colmode1.2013.11.014

Pagès , L., Collet, C., Drouet, J., Le Bot, J., Lecompte, F., \& Vercambre, G. (2004). Root Typ: a generic model to depict and analyse the root system architecture. Plant and Soil, 258, 109119. doi - 10.1023/B:PLSO.0000016540.47134.03

Phillips, C., Lambie, S., \& Marden, M. (2015). Observations of "coarse" root development in young trees of nine exotic species from a New Zealand plot trial. New Zealand Journal of Forestry Science, 45(1), 1-15. doi - 10.1186/s40490-015-0043-x

Pianosi, F., Beven, K., Freer, J., Hall, J., Rougier, J., Stephenson, D., \& Wagener, T. (2016). Sensitivity analysis of environmental models: A systematic review with practical workflow. Environmental Modelling \& Software, 79, 214-232. doi 10.1016/j.envs oft.2016.02.008

Randrup, T., Costello, R., \& McPherson, L. (2001). Tree root intrusion in sewer systems: Review of Extent and Costs. J. Infrastruct. Syst., 7(1), 26-31. doi 10.1061/(ASCE)1076-0342(2001)7:1(26)

Reddy, V.R., \& Pachepsky, Y. (2001). Testing a Convective-dispersive Model of Twodimensional Root Growth and Proliferation in a Greenhouse Experiment with Maize Plants. Annals of Botany, 87, 759-768. doi - 10.1006/anbo.2001.1409

Rigas, S., Ditengou, F., Ljung, K., Daras, G., Tietz, O., Palme, K., \& Hatzopoulos, P. (2013). Root gravitropism and root hair development constitute coupled developmental responses regulated by auxin homeostasis in the Arabidopsis root apex. New Phytologist, 197: 1130 1141. doi - 10.1111/nph.12092 
Runions, A., Lane, B. \& Prusinkiewicz, P. (2007). Modeling Trees with a Space Colonization Algorithm. Eurographics Workshop on Natural Phenomena.

Stava, O., Benes, B., Chen, B., Deussen, O., Kratt, S., Mech, J., \& Pirk, R. (2014). Inverse Procedural Modelling of Trees. Computer Graphics Forum, 33 (6), 118-131.

Stokes, A., Fourcaud, T., Cermak, J., Hruska, J., Nadyezdhina, N., Nadyezhdin, V., \& Praus, L. (2002). An evaluation of different methods to investigate root system architecture of urban trees in site: 1. Ground-Penetrating Radar. Journal of Arboriculture, 28(1), 2-10.

Stokes, A., \& Mattheck, C. (1996) Variation of wood strength in tree roots. Journal of Experimental Botany, 47, 693-699. doi - 10.1093/jxb/47.5.693

Stone, E., \& Kalisz, P. (1991). On the maximum extent of tree roots. Forest Ecology and Management, 46(1), 59-102. doi - 10.1016/0378-1127(91)90245-Q

Strom, S., Nathan, K., Woland, J. (2009). Site Engineering for Landscape Architects. (5 ${ }^{\text {th }}$ ed.). New Jersey: John Wiley and Sons.

Tobin, B., Čermák, J., Chiatante, D., Danjon, F., Di Iorio, A., Dupuy, L.,. Eshel, A., Jourdan, C., Kalliokoski, T., Laiho, R., Nadezhdina, N., Nicoll, B. , Pagès, L., Silva, J., \& Spanos, I. (2007). Towards developmental modelling of tree root systems. Plant Biosystems, 141 (3), 481-501. doi - 10.1080/11263500701626283

City of Toronto. Tree Protection Policy and Specifications for Construction Near Trees. July, 2016.

https://www1.toronto.ca/city_of_toronto/parks_forestry_recreation/urban_forestry/files/p df/TreeProtSpecs.pdf

University of Vienna, Computational Science Center. (n.d.). Rootbox, Retrieved June 6, 2017 from https://www.csc.univie.ac.at/rootbox/

Urban, J. (2008). Up by roots: Healthy Soils and Trees in the Built Environment. Champaign, Illinois: International Society of Arboriculture.

Wagner, B., Gärtner, H., Ingensand, H., \& Santini, S. (2011). A tool to model 3D coarse-root development with annual resolution. Plant Soil, 346, 79-96. doi - 10.1007/s 11104-011-07978

Walk, T., Jaramillo, R., Lynch, J. (2006). Architectural tradeoffs between adventitious and basal roots for phosphorus acquisition. Plant and Soil, 27, 347-366. doi - 10.1007/s 11104-0050389-6

Wu, Y., Cao, X., Chen, J., Cui, X., Guo, L., Lin, H. (2014). Ground-penetrating radar-based automatic reconstruction of three-dimensional coarse root system architecture. Plant Soil, 383, 155-172. doi - 10.1007/s 11104-014-2139-0

Xu, L., \& Mould, D. (2012) A procedural method for irregular tree models. Computers \& Graphics, 36, 1036-1047. doi - 10.1016/j.cag.2012.08.005 
Zobel, R., \& Waisel, Y. (2010). A plant root system architectural taxonomy: A framework for root nomenclature. Plant Biosystems, 144 (2), 507-512. doi - 10.1080/11263501003764483 